\title{
Acute Phase Proteins as Biomarkers of Disease: From Bench to Clinical Practice
}

\author{
Francisco J. Salgado, Pilar Arias, \\ Ana Canda-Sánchez and Montserrat Nogueira \\ University of Santiago de Compostela
}

Spain

\section{Introduction}

The term acute phase response (APR) refers to the inflammatory response of the host occurring shortly after the tissue injury. It comprises a wide variety of reactions started by different causes, like infection, tissue injury, burn, trauma, surgery, cancer or immunological disorders. These reactions aim to prevent ongoing tissue damage, isolate and eliminate the cause of the inflammation, and begin the repair process necessary to restore the normal function. Usually, the local response is accompanied by a systemic reaction characterized by the fast alteration of the concentrations of several plasmatic proteins, the APPs (Acute Phase Proteins) produced by the liver (Baumann \& Gauldie, 1994). In some diseases, the persistent immunological activation can cause chronic inflammation, often with pathological consequences. In other words, APR is a physiological condition occurring at the beginning of the inflammatory process and it is independent of the inflammation origin.

\subsection{The inflammatory response}

The inflammation is the ordered process mediated by the appearance of intercellular adhesion molecules on endothelia, and various inflammatory mediators released by tissue cells and leucocytes in response to tissue aggression; it is a protective response to injury or destruction of tissues, which serves to destroy, dilute, or wall off both the injurious agent and the injured tissues. It is the first reaction of the body under a situation of immunological stress: the innate non-specific response preceding specific immune reactions.

The term inflammation is purely descriptive and originally defined by the four Latin words dolor, rubor, calor and tumor (meaning pain, redness, warmth and swelling); these are the result of changes in the local blood vessels which in turn, lead to their dilatation and increased stickiness and permeability for passing leukocytes. Combined, the cell and fluid leakage into the tissue, as well as their local activities, account for the pain and swelling. The function of this inflammatory response is to bring serum molecules and immune cells to the damaged zone. In this response three principal components can be considered: i) increased blood supply, ii) increased capillary permeability and, iii) migration of cells out of the vessels and into the tissues.

In an inflammatory reaction there are implicated several cells of the immunological system and a great variety of response mediators. The uncontrolled migration of different 
leukocytes populations would make impossible the generation and control of the immune response. In this setting, the endothelium plays a key role. Endothelial cells are multifunctional cells that form a thin layer in the interior surface of blood vessels (Cines et al., 1998). They are responsible for maintaining the vascular homeostasis but, also, they are capable of secreting biologically active mediators when affected by infection, stress, hypertension, dyslipidemia or high homocysteine levels. The primary essential function of these endothelial cells is to regulate the permeability of the blood vessel and the exchange of fluids and cells between the blood and the surrounding tissue. In the inflammatory response they raise the expression of cell adhesion molecules that, therefore, increments the number of leukocytes binding endothelium (Pate et al., 2010). The increased permeability of the endothelium allows the migration of these cells, a process called extravasation, where they will exert its function. That is why they are so important for the recruitment and orchestration of an acute inflammatory response.

The role of the different cells and different interactions has been extensively described (Imhof \& Aurrand-Lions, 2004; Langer \& Chavakis, 2009; Ley et al., 2007; Schenkel et al., 2004; Vestweber, 2007), but briefly we are going to comment those. Normally, neutrophils are the first cells to appear at acute phase response, followed by macrophages (monocytes), lymphocytes (if there is an immunological challenge) and a small amount of basophils and eosinophils; if an extreme vascular leakage occurs, red blood cells may also be found. We can distinguish three steps for the migration of cell to the tissue during the inflammatory response (Butcher, 1991):

1-Receptor-mediated recruitment and adhesion to the endothelial cells: the regulation of the expression of selectin-P and selectin-E by the endothelium is key in this process (Lasky, 1995; Lawrence \& Springer, 1991). The expression of P-selectin is increased by inflammatory molecules such as histamine and thrombine, it is a weak binder to the leukocytes but causes them to "slow" their movement in the vessel. Selectin-E expression, on the other hand, it is upregulated by cytokines produced by injured cells, such as IL- 1 and TNF- $\alpha$; these cytokines also activate and increase the expression of integrins on the circulating leukocytes. Activated leukocytes, therefore, increase the affinity of their integrins for certain ligands on the endothelial surface, attaching them firmly to the endothelium.

2- Transmigration, or the movement of the activated leukocytes through the endothelium to reach the damaged tissue: the leukocytes adhered to the endothelium are stimulated by the chemokine gradient to move between the endothelial cells and across the basal membrane to the damaged area. Among the chemotactic factors we can find: i) products of the complement like C3a, C4a, C5a (Hartmann et al., 1997; Okusawa et al., 1988), ii) fibrin degradation products (FDPs) like the D-dimer (Gross et al., 1997), iii) prostaglandins (PGD2, PGE2) (Konya et al., 2010; Kuehn et al., 2011), iv) leukotrienes (LTB4) (Aked \& Foster, 1987) and chemokines like CCL5 (Kawai et al., 1999; Murooka et al., 2008) and IL-8 (Huber et al., 1991).

3- Movement of the activated leukocytes through the tissue: the binding to extracellular matrix proteins through the integrins and the CD44 protein allows these cells to arrive to the origin of the inflammation following the chemokine gradient (Kinashi, 2005).

\subsubsection{The Pro-inflammatory cytokines}

But in this local inflammatory process, activated leukocytes are not passive: in turn, they release cytokines and other mediators like glucocorticoids into the bloodstream, thus eliciting a widespread reaction in the organism. There are at least 15 cytokines known to be 
involved in this process. Some of them are positive/negative regulators of cell growth (IL-2, IL-3, IL-4, IL-7, IL-10, IL-11, IL-12 and GM-CSF), while others have got pro-inflammatory (TNF- $\alpha / \beta$, IL-1 $\alpha / \beta$, IL-6, IFN- $\alpha / \gamma$, IL-8) or anti-inflammatory activities (IL-1 antagonists, soluble IL-1R, IL-1 and TNF- $\alpha$ binding protein)(van Miert, 1995).

Proinflammatory cytokines, released mainly by activated macrophages, are crucial for the induction of other cytokines (IL-6, IL-8), while agents such as platelet activating factor, prostaglandins, leukotrienes and nitric oxide increase the chemotactic gradient and therefore the leukocyte recruitment to the inflammation site. They are also responsible for the fever and catabolism of muscle proteins through their action at the CNS level: We will briefly comment some carachteristics of the proinflammatory cytokines involved in the acute phase reaction and the production of APPs:

- Tumor necrosis factor-alpha (TNF-a)

TNF- $\alpha$ is the main cytokine that mediates acute inflammation. TNF is produced by monocytes, macrophages, dendritic cells, Th1 cells, and other cells. Some of its functions include: to stimulate the cells from the endothelium to produce selectins and the leukocytes to express integrins, to activate the coagulation pathway, to increase the production of chemokines in endothelial cells and macrophages, to activate neutrophils and their killer activity, etc (Baud \& Karin, 2001). It is also responsible for stimulating the liver to produce APPs (Xanthoulea et al., 2004), and acting on muscles (Collins \& Grounds, 2001) and fat (Lu et al., 2006; Plomgaard et al., 2008; Rydén et al., 2002) to stimulate catabolism for energy conversion. In addition, it interacts with the hypothalamus to induce lack of appetite (Tracey et al., 1990), fever (Rothwell \& Hopkins, 1995; Stefferl et al., 1996) and sleep (Deboer et al., 2002; Fang et al., 1997). It also participates in the cicatrization process through the stimulation of collagen and collagenase synthesis (Theiss et al., 2005).

-Interleukin-1 (IL-1)

IL-1 function is similar to TNF- $\alpha$ as it also mediates acute inflammatory responses, and together they enhance the inflammatory response (Dinarello \& Wolff, 1993; Vassalli, 1992). Monocytes, macrophages, dendritic cells and endothelial cells are the main sources of IL-1. Functions of IL-1 include the promotion of the inflammation through the activation of the coagulation pathway and the synthesis of adhesion factors on endothelial cells and leukocytes (Bevilacqua et al., 1984; Nawroth et al., 1986) and the activation of macrophages (Dinarello, 1988). It is also responsible for stimulating the liver to produce APPs (Baumann \& Gauldie, 1994; Mortensen et al., 1988; Prowse \& Baumann, 1989), for activating the catabolism of the fat tissue for energy conversion (Feingold et al., 1992; Tocco-Bradley et al., 1987); and for regulating the synthesis of collagen and collagenase for scar tissue formation (Duncan \& Berman, 1989; Mizel et al., 1981).

It is also noteworthy the role that IL-1 plays in the induction of the fever and sleep (Krueger et al., 1984; Opp \& Krueger, 1991), as well as the stimulatory effect of IL-1 on the pituitaryadrenal axis in the CNS (Besedovsky et al., 1986; Sapolsky et al., 1987), thus making it responsible for the control of food intake (Luheshi et al., 1999) during the acute phase response.

Interleukin-1 and TNF- $\alpha$ have also being linked to the depression and sickness behaviour associated to the inflammatory response (Dantzer, 2001; Konsman et al., 2002). One theory behind this effect it is the role of IL-1 over serotoninergic neurons, where IL-1 could activate the serotonin transporter directly. This could lead to the removal of serotonin from the synaptic cleft, thus generating a serotoninergic deprivation signal together with the tryptophan deprivation derived from the APPs production (Leonard \& Song, 1999). 
- Interleukin-6 (IL-6)

IL-6 stimulates the liver to produce APPs and it is released by many cells including Tlymphocytes, macrophages, monocytes, endothelial cells, and fibroblasts (Prowse \& Baumann, 1989). It is also responsible for the proliferation stimulation of B-lymphocytes (Muraguchi et al., 1988; Taga et al., 1987) and increases the neutrophil activity (Brom \& König, 1992).

The outcome of the inflammation will depend on the tissue as well as the extent of injury and the injurious agent that has caused it. Usually, the resolution will bring the inflamed tissue to its status prior to the injury but, some times, the inflammation is not resolved properly. Sometimes a persistent damage or an incomplete resolution process can produce a chronic inflammation due to the continuous production of pro-inflammatory cytokines. Often, the reason behind the abnormal inflammatory response is not known, and it is the reason behind many human diseases. Some of them have got an immunological origin like the allergic reactions or myopathies (Lundberg \& Grundtman, 2008); but sometimes the origin is non-immunological like in rheumatoid arthritis, cancer (Coussens \& Werb, 2002), atherosclerosis (Libby, 2002), ischaemic heart disease (Maseri et al., 1996), inflammatory bowel diseases (Fiocchi, 1998), etc (many articles, reviews and books cover these topics, so this is not an extensive list). In all of the above mentioned disorders we can also see alterations in the levels of different APPs produced by the liver; the next section will present an extensive list of these proteins, where the main characteristics of the different groups will be commented briefly.

\section{Acute phase proteins}

APPs is a group of plasma proteins whose concentration varies in response to inflammation (Baumann \& Gauldie, 1994). They are synthesized mainly by the liver but also by other cell types (monocytes, endothelial cells, fibroblasts and adipocytes). According to the variations in the concentration of these proteins they can be classified in positive APPs, those that increase the concentration, and negative APPs, those that show decreased concentrations upon the inflammatory response. There are also other sub-classifications for the positive APPs, according to the variation in concentration: Group I positive APPs increase up to $50 \%$ (ceruloplasmin and complement factor-3 (C3)), Group II from 2 to 5 times (haptoglobin, fibrinogen, a-globulins with antiprotease-activity and lipopolysaccharide binding protein), and Group III from 5 to more than 1000 times (CRP and SAA) (Dowton \& Colten, 1988).

There is a lot of diversity regarding the timings that each of these proteins show any variation on its levels. Some proteins alter their levels as soon as $4 \mathrm{~h}$, while others can take up to $24 \mathrm{~h}$ or even days. CRP (C-reactive protein) and SAA (serum amyloid A) are some of these very rapidly increasing proteins: they elevate their levels within the first $4 \mathrm{~h}$ after tissue injury, opposite to the lipopolysaccharide binding protein, that requires at least $8 \mathrm{~h}$, or the serum alpha-l-acid glycoprotein, that needs $48 \mathrm{~h}$. Some of these proteins remain elevated for various days, while other only for few hours. The origin behind the inflammation is also important: the plasma fibrinogen shows a peak in the concentration at $24 \mathrm{~h}-48 \mathrm{~h}$ or at $96 \mathrm{~h}$, depending on the inflammatory stimulus (Colley et al., 1983; Gruys et al., 2005)

As commented before TNF- $\alpha$, IL-1, and IL- 6 are the main cytokines involved in the hepatocytic secretion of APPs, but there are many others. Indeed, all type of combinatorial effects can regulate the hepatocytic secretion of an APP (addition, synergy, antagonism). IL1 and IL-6 have been used to classify APPs into two subgroups: Type 1 requires the 
synergistic effect of both interleukins for maximum synthesis (examples: CRP, SAA, Alpha1-acid glycoprotein 1); type 2 are those that only require IL-6 and usually IL-1 acts suppressing more than enhancing its production (examples: fibrinogen chains, haptoglobin, alpha-2-Macroglobulin (Ramadori \& Christ, 1999)). There are some cytokines that regulate the expression of one or two APPs, like Activin A, a cytokine related with the superfamily of TGF- $\beta$. On the other hand, other APPs are the result of a wider combination of cytokines. That is the case of SAA that requires the synergitic effect of bacterial LPS and several cytokines (mainly IL-1, IL-6, and TNF, but also LIF, CNTF, oncostatin M, IL-11, and cardiotrophin-1) to be produced (Benigni et al., 1996).

The main structural characteristics of APPs can be found in Table 1. Regarding their functions, summarized in Table 2, they are opsonisation and elimination of microorganisms and their products (CPR), activation of the complement system (complement C4a, C4b) and the lectin complement pathway (manan-binding lectin), inhibition of certain enzymes (alpha-1-antitrypsin, alpha-2-antiplasmin) or interleukins (interleukin-1 receptor antagonist protein), blockade of free radicals and/or elimination of haemoglobin residues (haptoglobin, serotransferrin, hemopexin), regulation of the immune response (serum amyloid P), coagulation and tissue repair/remodelling (fibrinogen, plasminogen), transport of ions (apolipoproteins, calcitonin precursor) and hormones (serum albumin), etc. Others, like alpha 2-macroglobulin and the coagulation factors, also play an important role in wound healing, because they collaborate with the immune system by increasing the vascular permeability, because they have chemotactic properties, or even because they are able of trapping pathogens in local blood clots.

\section{Acute phase proteins currently used in routine clinical practice}

As compared with cytokine and other short distance mediators of cellular responses production, which occurs in pulsed patterns cleared from the circulation within a few hours due to their small size, the native molecular mass of APPs secreted by the liver is larger than the kidney filtration cutoff (about $45 \mathrm{kDa}$ ). An example is albumin, which is just larger and has a lifetime of about 21 days. This fact, together with their large half-life, ensures an extended residence time in plasma, which means that levels of some APPs may remain unchanged for days and may be useful for diagnostic purposes. Currently, alterations in levels of some APPs are used to assess either the health/nutritional status of an individual or, mainly, to check for the presence of reactive processes, which has more advantages than measuring IL-6, a sensitive indicator of inflammation or infection but with a normal range of $0-5 \mathrm{pg} / \mathrm{ml}$ and short plasma clearing times. An analysis of US Food and Drug Administration (FDA) approvals for IVD assays aimed to the quantitative detection of acute phase reactants reveals about 80 companies involved (Table 3), of which 15 dominate the market: Abbott Laboratories, Beckman Coulter, Dade Behring, Diasorin, Globalemed, Kamiya Biomedical, Kent Laboratories, Nitto Boseki, Olympus Life Science Research, OrthoClinical Diagnostics, Polymedco, Randox Laboratories, Roche Diagnostics, Siemens Medical Solutions Diagnostics, and The Binding Site.

About $51 \%$ of FDA-cleared or approved IVD assays are aimed to the detection of unique plasma or serum proteins that carry out their normal function in plasma. Most of these proteins are negative or positive acute phase reactants, like albumin, C-reactive protein, $\alpha 1$ antitrypsin, transferrin, ceruloplasmin, or fibrinogen. Table 4 summarizes different tests from the companies listed in Table 3 intended to the detection of APPs in clinical practice. 


\begin{tabular}{|c|c|c|c|c|c|c|c|c|}
\hline : & 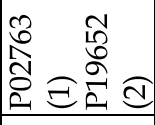 & $\begin{array}{l}\vec{\sigma} \\
0 \\
0 \\
0 \\
\end{array}$ & $\begin{array}{l}\hat{\sigma} \\
\text { o } \\
0 \\
0\end{array}$ & $\begin{array}{l}\infty \\
8 \\
0 \\
0 \\
\end{array}$ & $\begin{array}{l}8 \\
\text { ठे } \\
\text { ○े } \\
\text { \& }\end{array}$ & $\begin{array}{l}\text { जิ } \\
\text { કै } \\
\text { \& }\end{array}$ & $\begin{array}{l}\text { Lి } \\
\text { ลे } \\
\text { \& } \\
\end{array}$ & 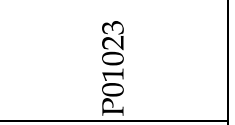 \\
\hline 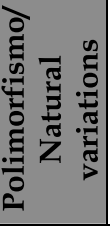 & 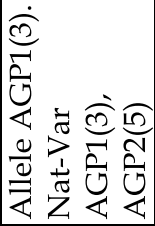 & 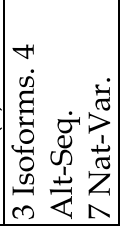 & $\begin{array}{l}\frac{\pi}{2} \\
\frac{1}{\pi} \\
\frac{\pi}{2} \\
\infty\end{array}$ & $\begin{array}{l}\tilde{\pi} \\
z_{1}^{1} \\
\pi \\
z \\
\sigma \\
\sigma\end{array}$ & 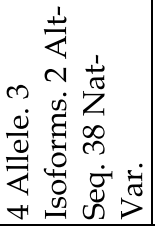 & 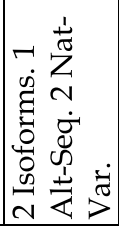 & $\begin{array}{l}\vec{\pi} \\
\frac{1}{\pi} \\
\frac{1}{Z} \\
10\end{array}$ & 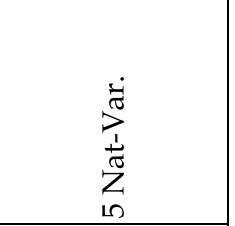 \\
\hline 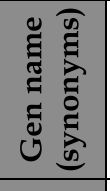 & 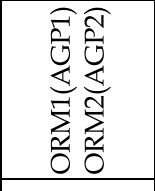 & 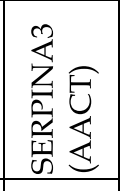 & 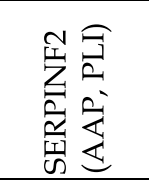 & 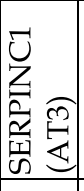 & 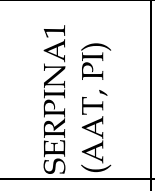 & $\begin{array}{l}0 \\
9 \\
\\
\end{array}$ & 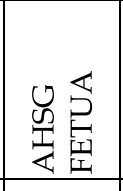 & 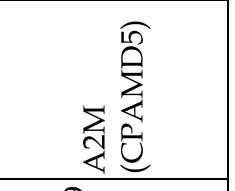 \\
\hline$\sum_{\substack{Z \\
\vdots}}^{Z}$ & 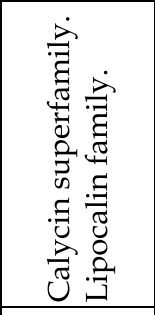 & 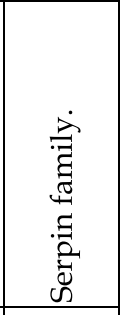 & 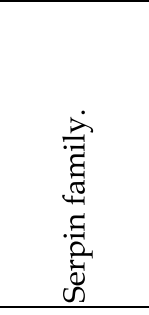 & 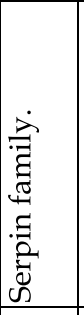 & 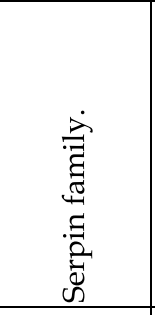 & 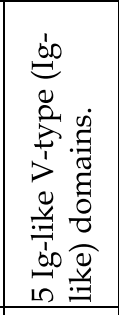 & 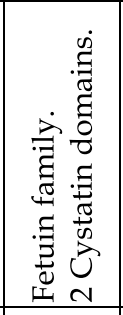 & 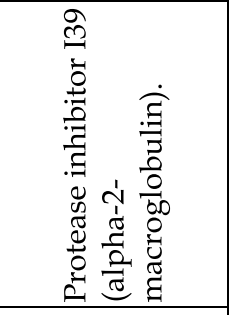 \\
\hline 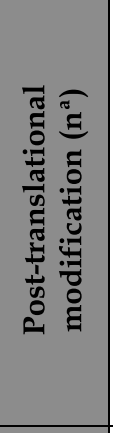 & 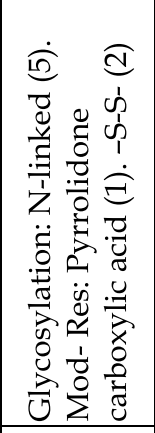 & 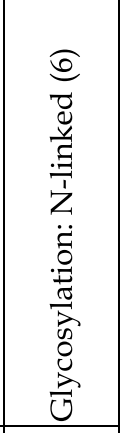 & 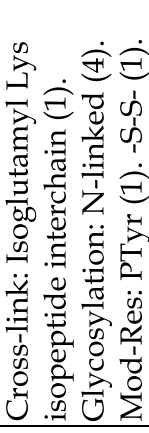 & 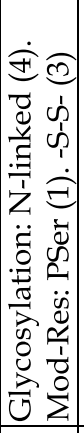 & 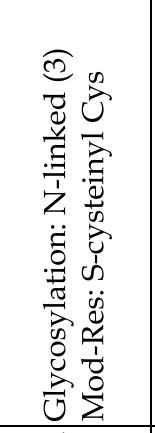 & 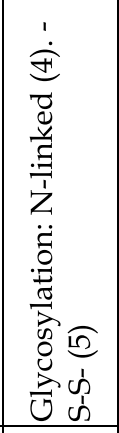 & 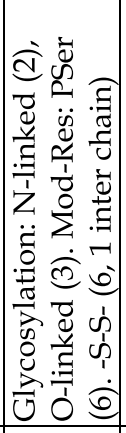 & 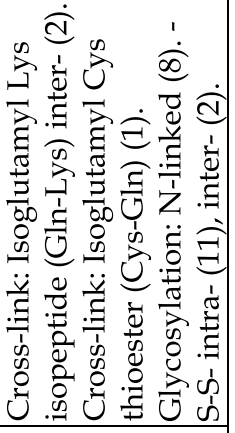 \\
\hline$\sum_{\substack{\pi \\
0}}^{+}$ & 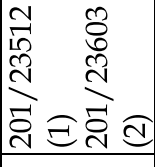 & 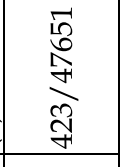 & 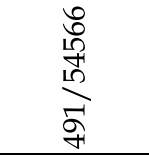 & 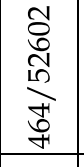 & $\begin{array}{l}\hat{N} \\
\hat{\sigma} \\
\begin{array}{l}1 \\
\infty \\
0 \\
F\end{array} \\
\end{array}$ & 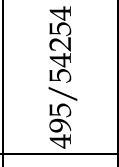 & 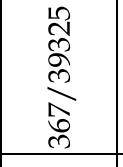 & 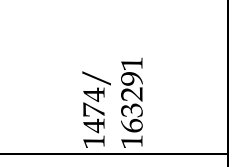 \\
\hline 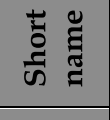 & 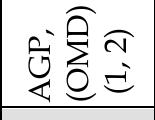 & U & 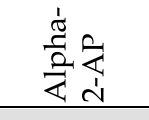 & 宣 & 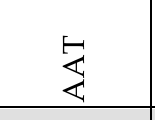 & $\begin{array}{l}0 \\
\end{array}$ & 先 & ২্য \\
\hline 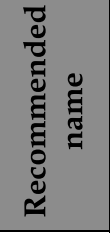 & 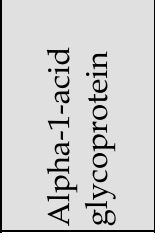 & 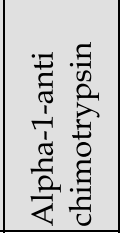 & 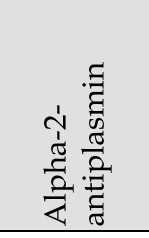 & 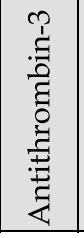 & 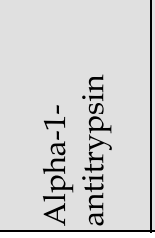 & 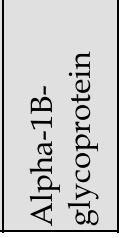 & 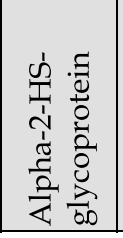 & 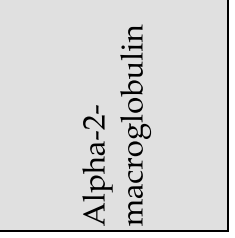 \\
\hline
\end{tabular}




\begin{tabular}{|c|c|c|c|c|c|c|c|c|}
\hline 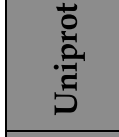 & $\begin{array}{l}\text { fै } \\
\text { ठิ } \\
\text { \& }\end{array}$ & 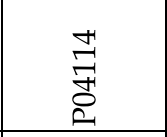 & 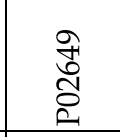 & $\begin{array}{l}\text { ఫิ } \\
\text { ลิ } \\
\text { \& }\end{array}$ & 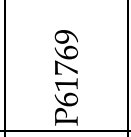 & 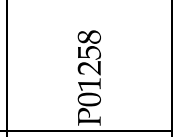 & $\begin{array}{l}\text { ధ } \\
\text { \&̊ } \\
8 \\
\end{array}$ & $\begin{array}{l}10 \\
\text { 85 } \\
8 \\
\&\end{array}$ \\
\hline 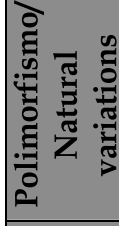 & 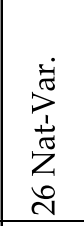 & 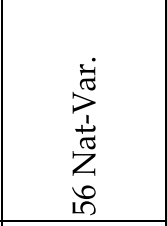 & 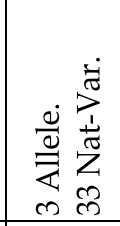 & 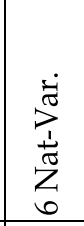 & $\begin{array}{l}\dot{\vec{\sigma}} \\
\stackrel{i}{1} \\
\frac{\pi}{\pi} \\
\end{array}$ & 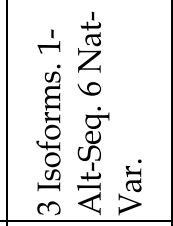 & 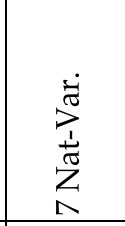 & 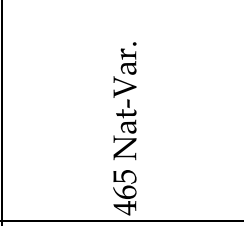 \\
\hline 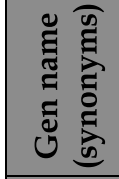 & $\begin{array}{l}\text { 离 } \\
0 \\
8\end{array}$ & $\begin{array}{l}n \\
0 \\
\text { \& }\end{array}$ & $\begin{array}{l}\text { 11 } \\
\text { \& } \\
\end{array}$ & $\begin{array}{l}1 \\
0 \\
8\end{array}$ & 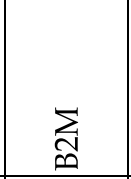 & 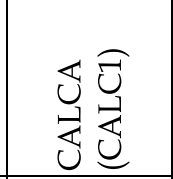 & $\hat{U}$ & 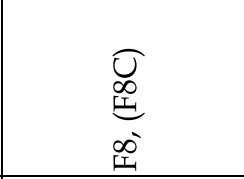 \\
\hline$\sum_{\substack{Z \\
\vdots}}^{Z}$ & 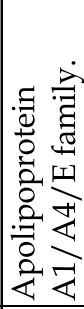 & 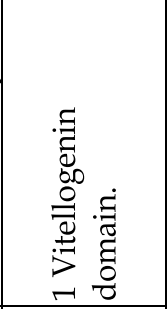 & 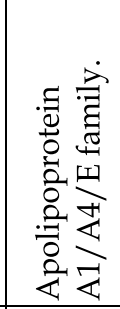 & & 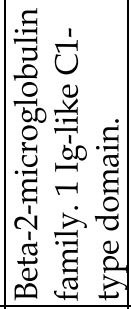 & 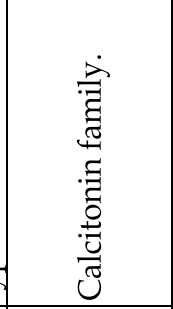 & 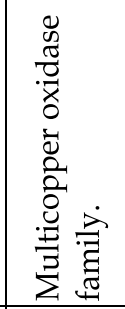 & 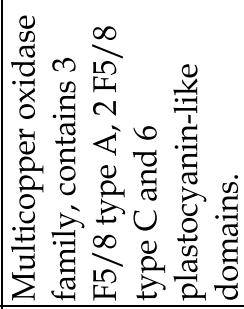 \\
\hline 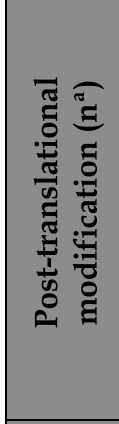 & 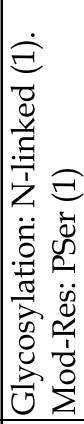 & 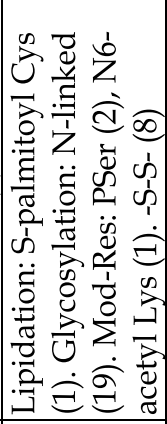 & 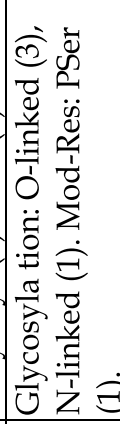 & 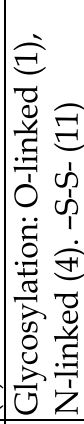 & 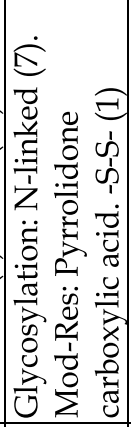 & 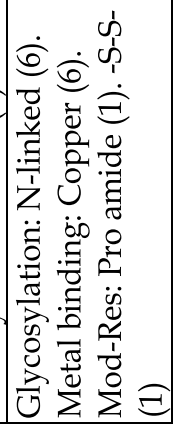 & 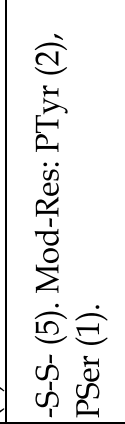 & 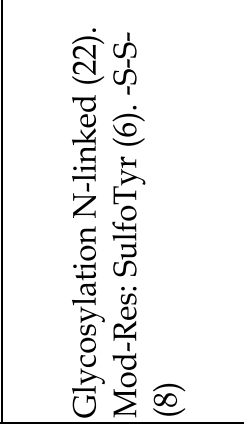 \\
\hline 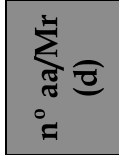 & 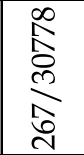 & 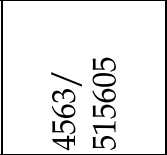 & 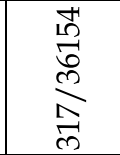 & 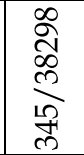 & $\begin{array}{l}\stackrel{10}{1} \\
\stackrel{2}{=} \\
\stackrel{\sigma}{\sigma} \\
\stackrel{\sigma}{=}\end{array}$ & 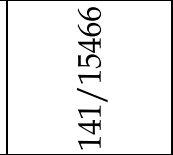 & 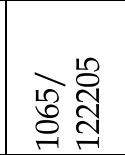 & 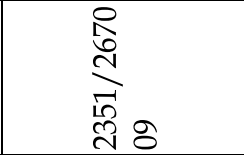 \\
\hline 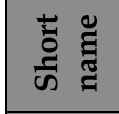 & $\begin{array}{l}\text { Z } \\
8 \\
0 \\
\end{array}$ & 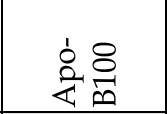 & 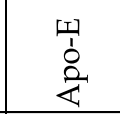 & О్ & 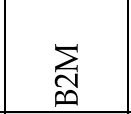 & 它忘式 & 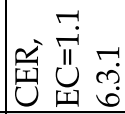 & 空 \\
\hline 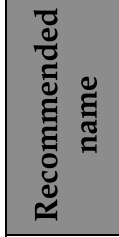 & 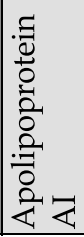 & 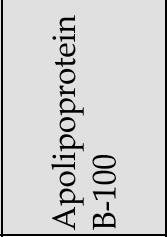 & 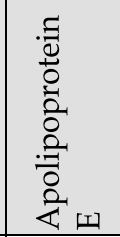 & 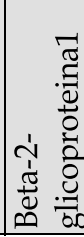 & 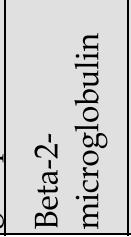 & 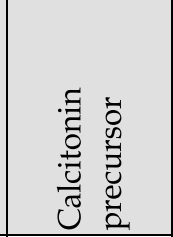 & 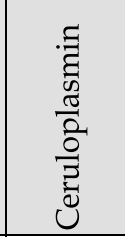 & 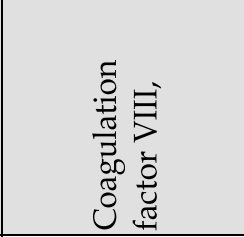 \\
\hline
\end{tabular}




\begin{tabular}{|c|c|c|c|c|c|c|}
\hline 芫 & $\begin{array}{l}\sqrt[\infty]{0} \\
8 \\
0 \\
8\end{array}$ & $\begin{array}{l}\text { d } \\
\text { Oे } \\
\vdots \\
\&\end{array}$ & $\begin{array}{l}\text { H } \\
8 \\
8\end{array}$ & $\begin{array}{l}n \\
0 \\
8 \\
8\end{array}$ & 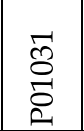 & $\begin{array}{l}\text { 我 } \\
\text { సิ } \\
\text { \& }\end{array}$ \\
\hline 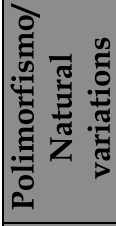 & $\begin{array}{l}\sum_{0}^{ \pm} \\
\frac{1}{\pi} \\
0\end{array}$ & 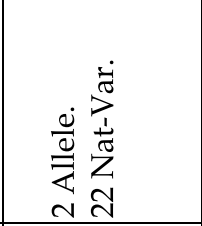 & 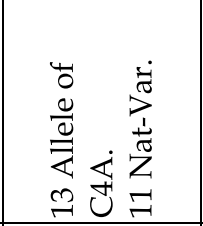 & 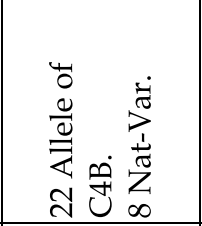 & 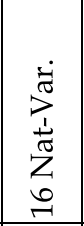 & 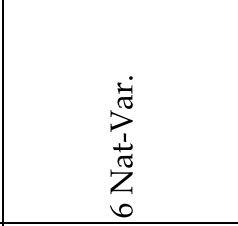 \\
\hline 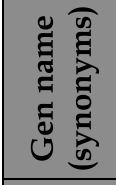 & U & 它志 & せ & 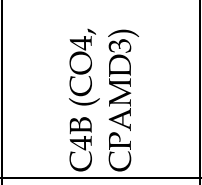 & 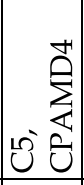 & 8 \\
\hline Z & 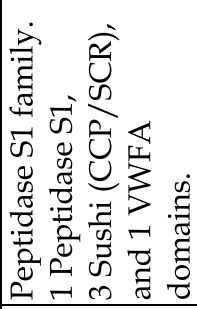 & 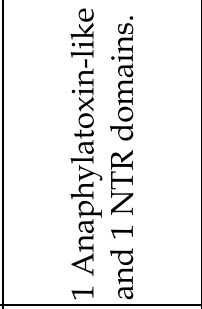 & 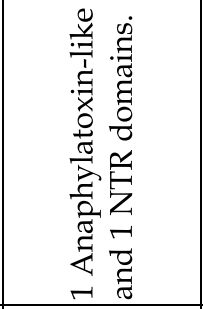 & 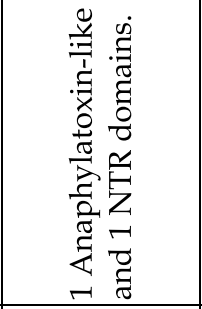 & 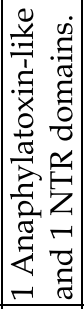 & 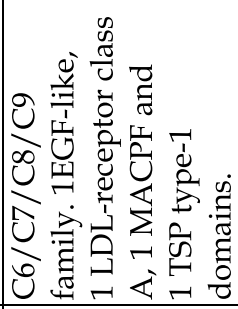 \\
\hline 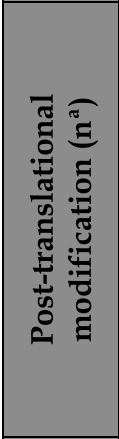 & 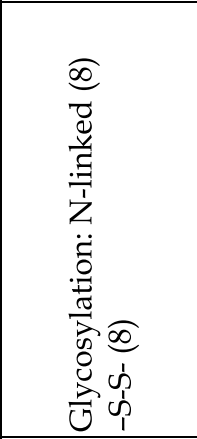 & 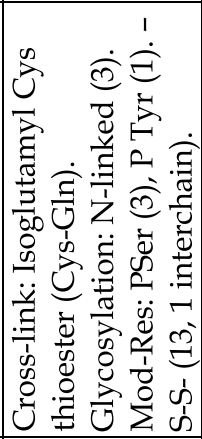 & 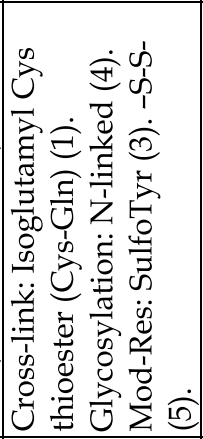 & 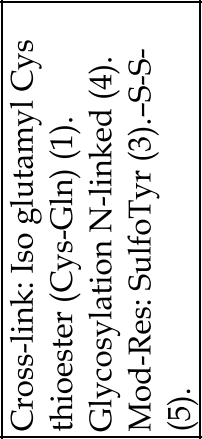 & 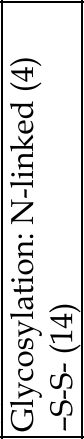 & 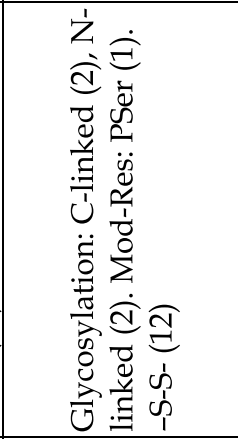 \\
\hline 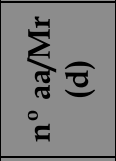 & 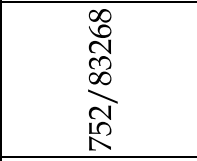 & तે & 吉 & 吉令 & $\begin{array}{|cc|} & 0 \\
0 & 0 \\
0 & 0 \\
0 & \infty \\
-1 & 0 \\
& -1 \\
\end{array}$ & 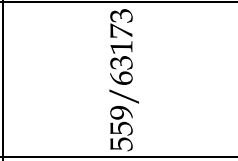 \\
\hline 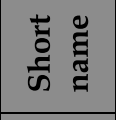 & 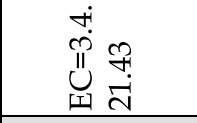 & $\mathcal{U}$ & u & 光 & 0 & 8 \\
\hline 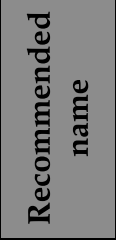 & 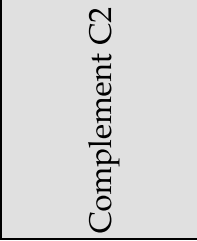 & 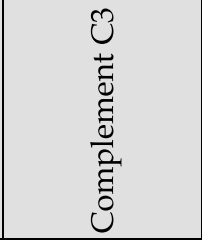 & है & 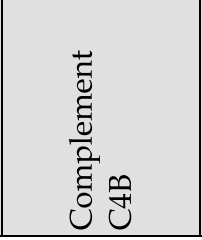 & 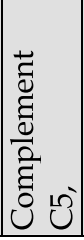 & हूँ \\
\hline
\end{tabular}




\begin{tabular}{|c|c|c|c|c|c|c|}
\hline 范 & 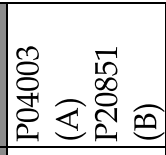 & $\begin{array}{l}10 \\
\infty \\
\infty \\
0 \\
0\end{array}$ & 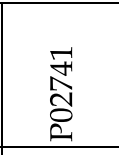 & $\begin{array}{l}\sqrt[1]{n} \\
\text { ○े } \\
\text { q }\end{array}$ & 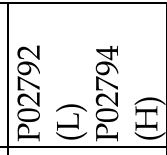 & 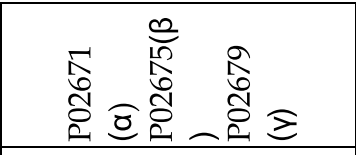 \\
\hline 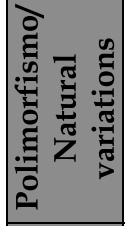 & 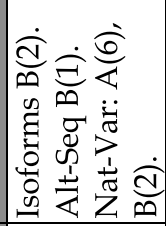 & $\begin{array}{c}\dot{\vec{z}} \\
\frac{1}{\pi} \\
\frac{\pi}{Z} \\
m \\
m\end{array}$ & 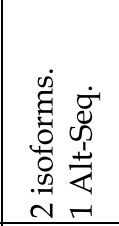 & 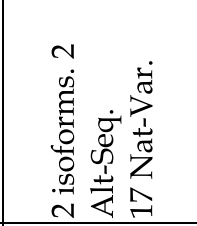 & 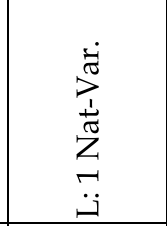 & 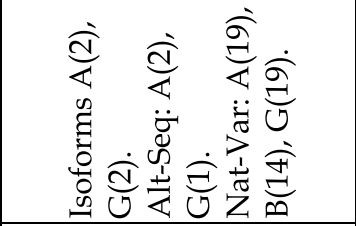 \\
\hline 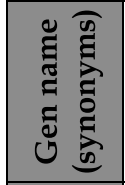 & 离 & 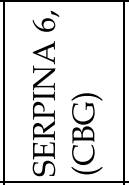 & 总氞 & 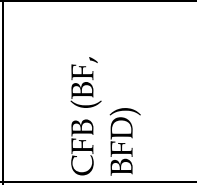 & 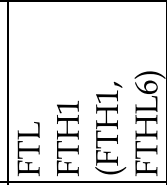 & 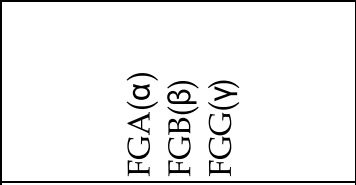 \\
\hline 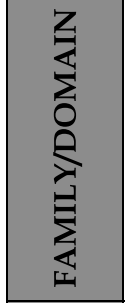 & 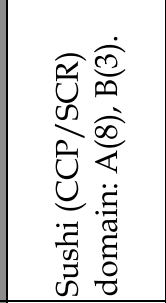 & 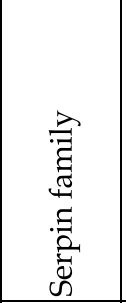 & 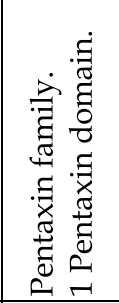 & 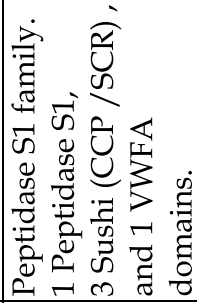 & 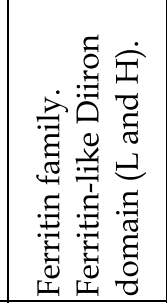 & 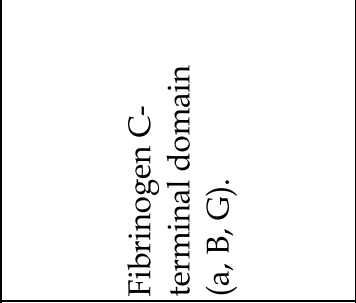 \\
\hline 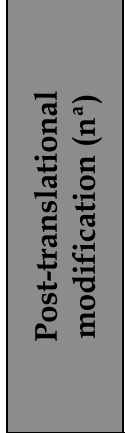 & 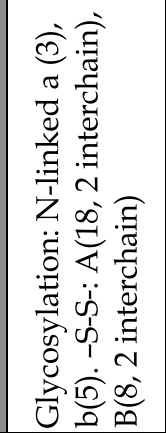 & 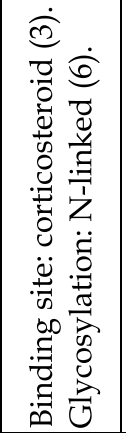 & 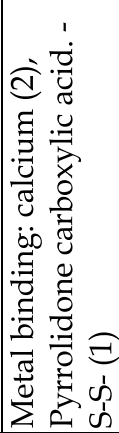 & 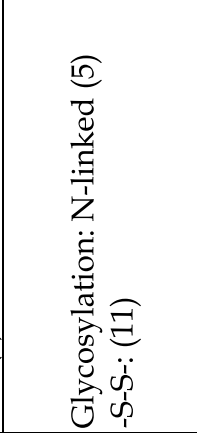 & 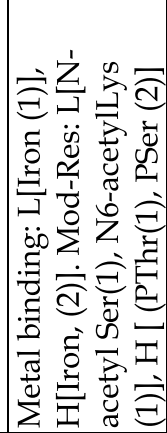 & 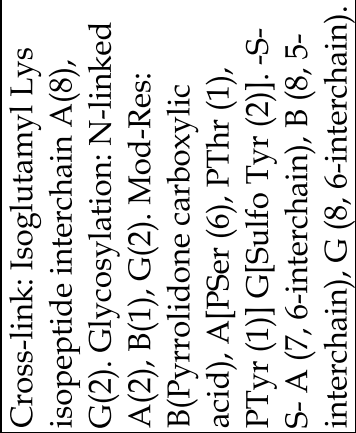 \\
\hline$\sum_{\substack{J \\
0 \\
j}}^{H}$ & 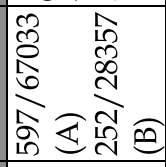 & 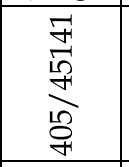 & 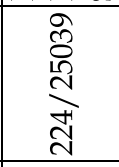 & 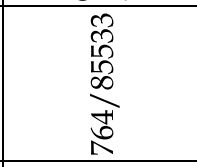 & 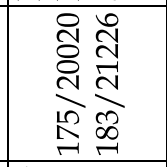 & 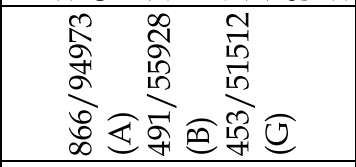 \\
\hline 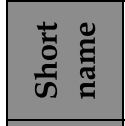 & 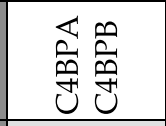 & U & 穵 & 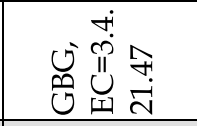 & 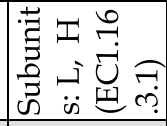 & 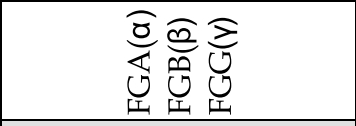 \\
\hline 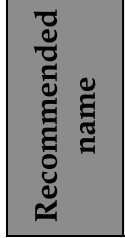 & 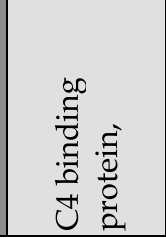 & 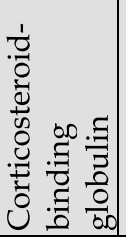 & 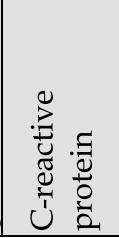 & 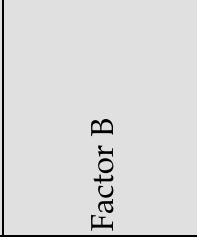 & 吾 & 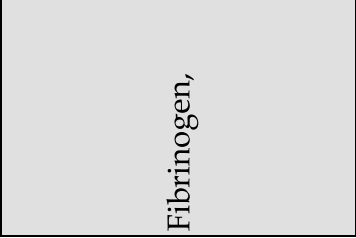 \\
\hline
\end{tabular}




\begin{tabular}{|c|c|c|c|c|c|c|c|}
\hline 范 & $\begin{array}{l}\text { مิ } \\
\text { ลิ } \\
\text { \& }\end{array}$ & 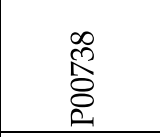 & $\begin{array}{l}\text { के } \\
\text { हू } \\
\text { ळે }\end{array}$ & 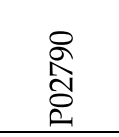 & 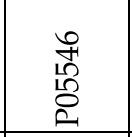 & 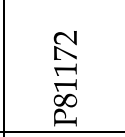 & 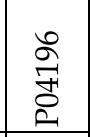 \\
\hline 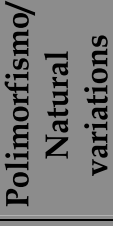 & 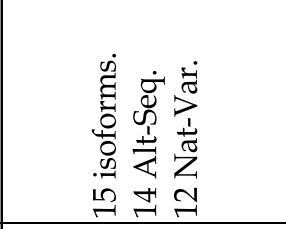 & $\frac{d}{\stackrel{a}{*}}$ & 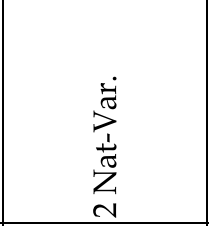 & 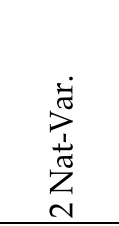 & $\begin{array}{l}\text { ते } \\
\frac{1}{\pi} \\
\bar{\pi} \\
a\end{array}$ & 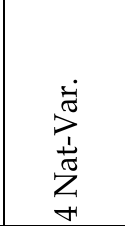 & 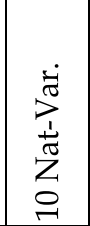 \\
\hline 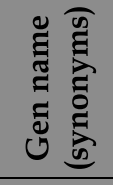 & 兊疍 & 臭 & 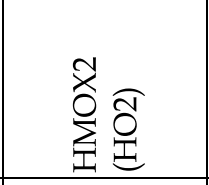 & 死 & 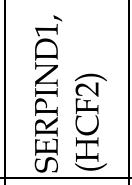 & 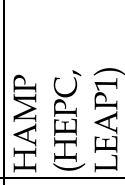 & 全 \\
\hline 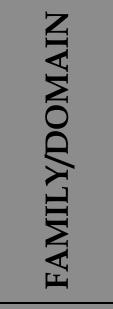 & 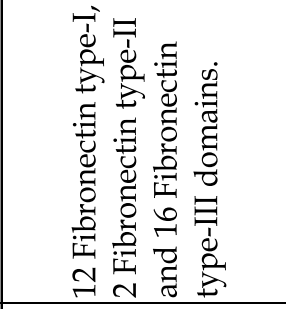 & 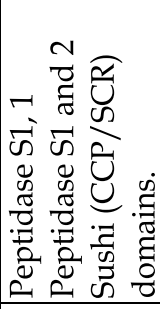 & 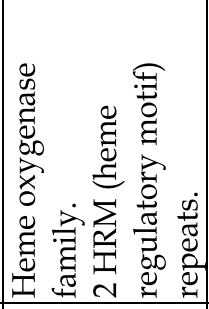 & 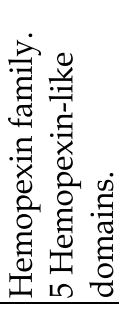 & 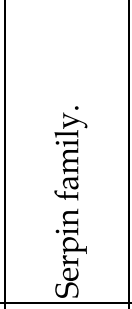 & 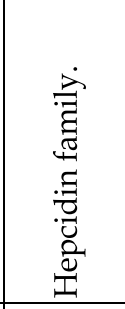 & 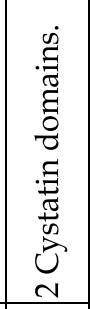 \\
\hline 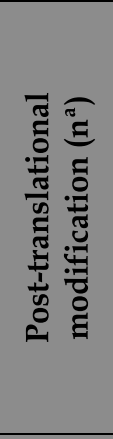 & 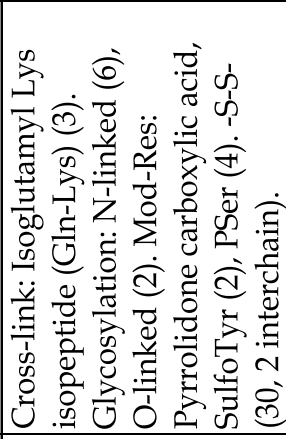 & 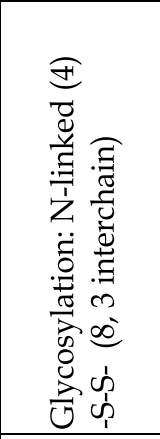 & 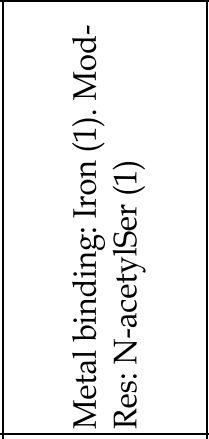 & 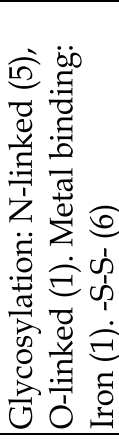 & 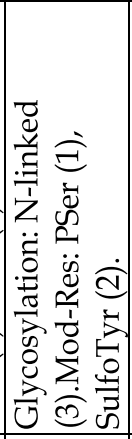 & $\begin{array}{l}\text { ङ } \\
\text { p. } \\
\text { 1 } \\
1\end{array}$ & 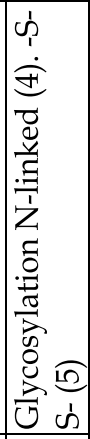 \\
\hline 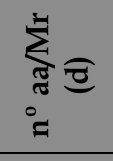 & 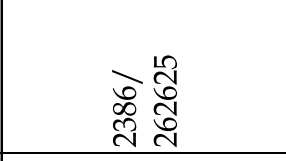 & 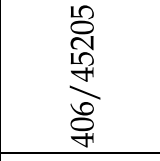 & 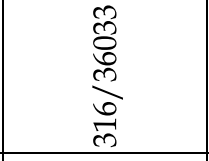 & 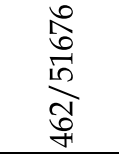 & 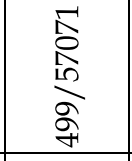 & 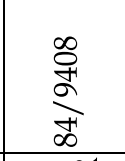 & 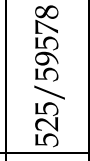 \\
\hline 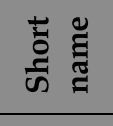 & 胥 & $\begin{array}{l}\overparen{a} \\
\stackrel{0}{\sigma}\end{array}$ & 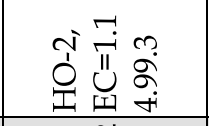 & 齐 & 氙泴 & 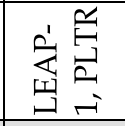 & $\begin{array}{l}0 \\
\underline{n} \\
\text { 王 }\end{array}$ \\
\hline 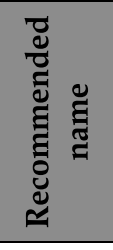 & 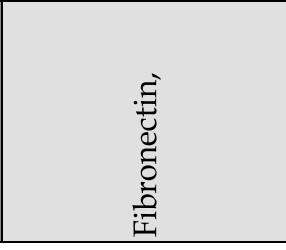 & 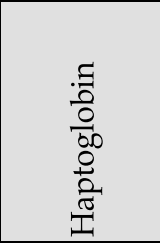 & 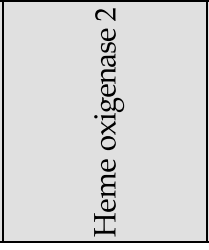 & 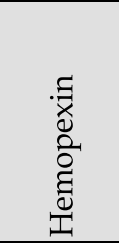 & 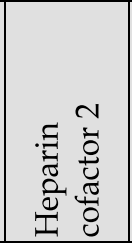 & 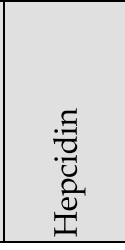 & 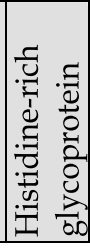 \\
\hline
\end{tabular}




\begin{tabular}{|c|c|c|c|c|c|c|}
\hline : & 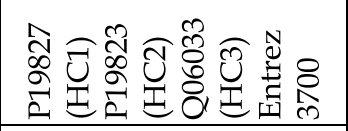 & $\begin{array}{l}0 \\
\stackrel{1}{1} \\
\infty \\
\infty \\
\\
\end{array}$ & 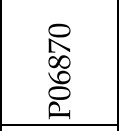 & $\begin{array}{l}\text { ڤి } \\
\text { ลิ } \\
\text { ¿ิ } \\
\text { \& }\end{array}$ & $\stackrel{\substack{\mathbb{N} \\
\infty \\
\Sigma}}{\Sigma}$ & $\begin{array}{l}\text { શิ } \\
\text { F } \\
\text { \& }\end{array}$ \\
\hline 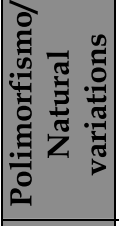 & 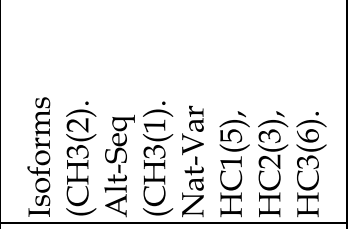 & 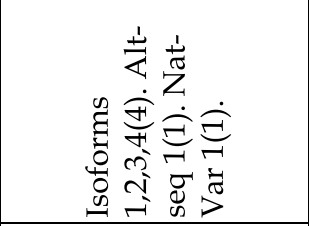 & 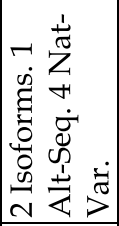 & 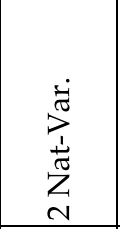 & 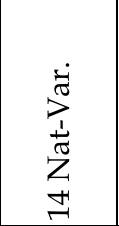 & 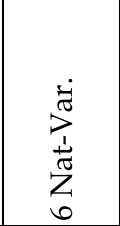 \\
\hline 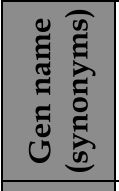 & 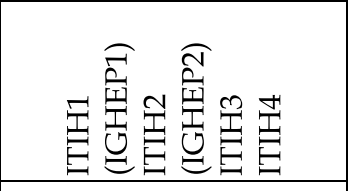 & 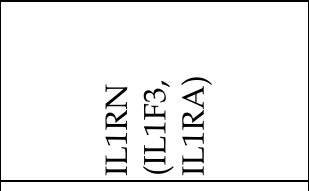 & & 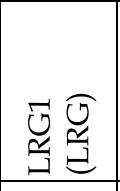 & 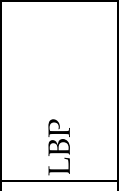 & กิ \\
\hline 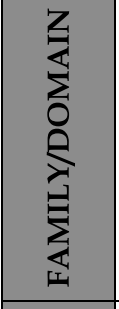 & 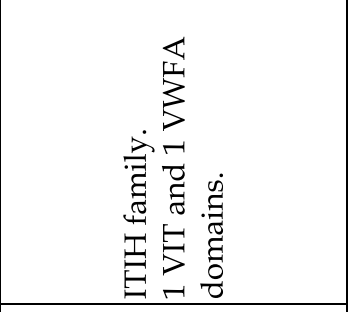 & 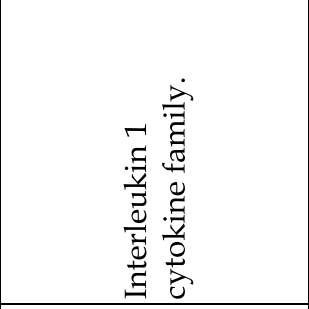 & 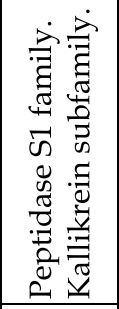 & 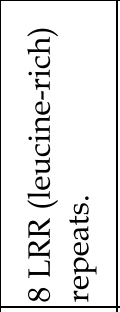 & 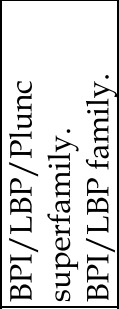 & 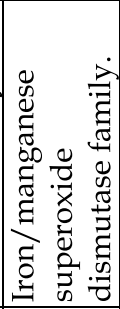 \\
\hline 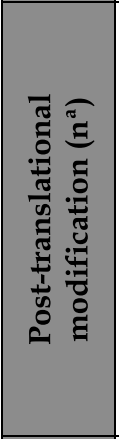 & 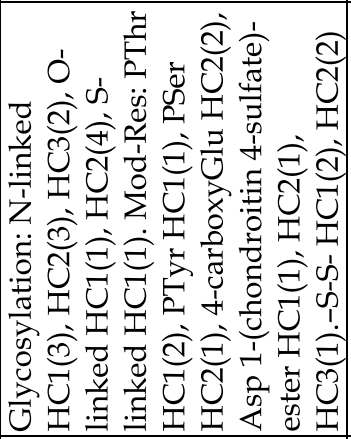 & 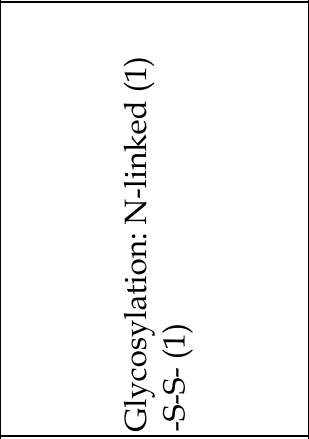 & 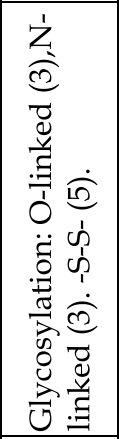 & 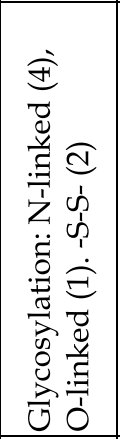 & 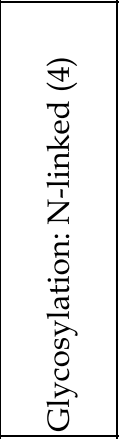 & 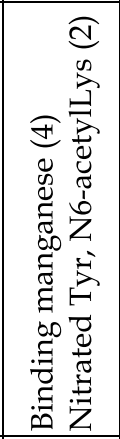 \\
\hline 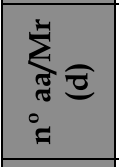 & 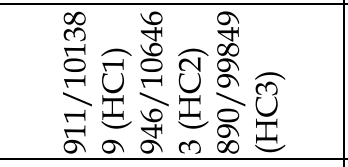 & 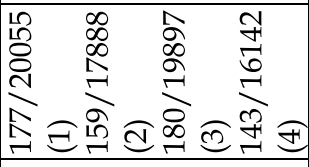 & 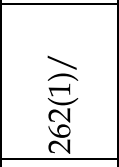 & 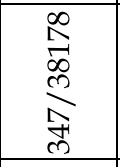 & 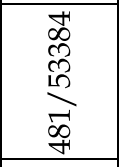 & 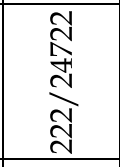 \\
\hline 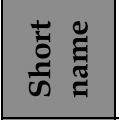 & 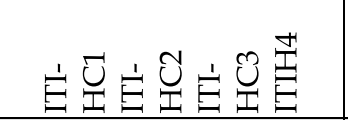 & 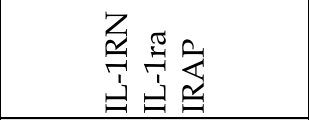 & $\frac{\overrightarrow{4}}{\vec{y}}$ & $\underset{\mathscr{G}}{\mathscr{G}}$ & 魚 & تُّ \\
\hline 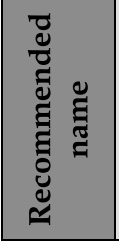 & 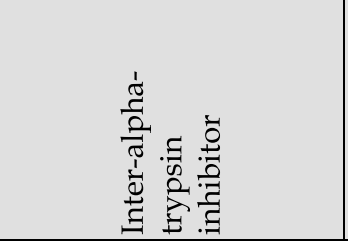 & 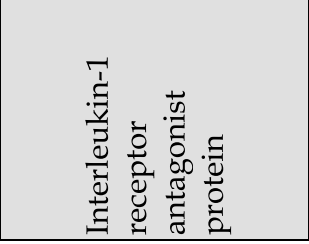 & 离 & 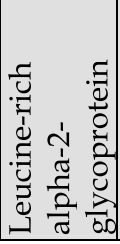 & 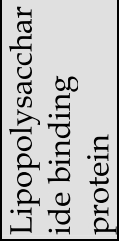 & 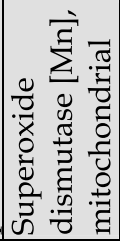 \\
\hline
\end{tabular}




\begin{tabular}{|c|c|c|c|c|c|c|c|}
\hline : & $\begin{array}{l}\text { స్ } \\
\stackrel{\Xi}{\Xi}\end{array}$ & $\begin{array}{l}\infty \\
10 \\
1 \\
0 \\
0 \\
1\end{array}$ & $\begin{array}{l}10 \\
10 \\
10 \\
0 \\
0\end{array}$ & $\begin{array}{l}\text { 吉 } \\
\text { 今8 } \\
\text { \& }\end{array}$ & $\begin{array}{l}\sqrt[7]{7} \\
\stackrel{10}{0} \\
0\end{array}$ & 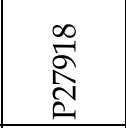 & $\begin{array}{l}\text { ๖े } \\
\text { ิิ } \\
\text { ฉิ }\end{array}$ \\
\hline 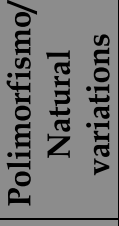 & 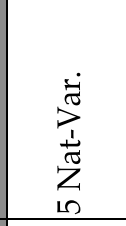 & & 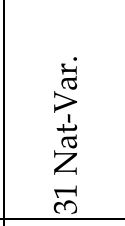 & 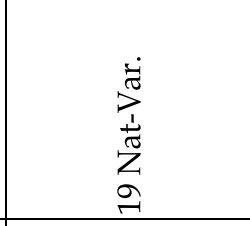 & 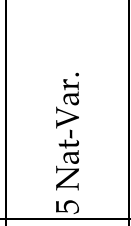 & $\begin{array}{l}\pi \\
i_{1}^{1} \\
\frac{1}{\pi} \\
\infty \\
\infty\end{array}$ & \\
\hline 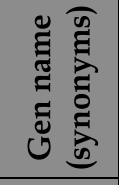 & 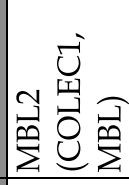 & 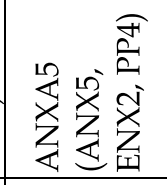 & 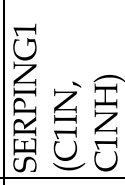 & $\begin{array}{c}0 \\
\underline{a} \\
\end{array}$ & 焉 & 穵苞 & 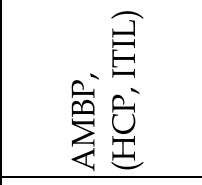 \\
\hline 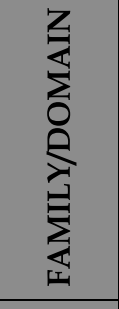 & 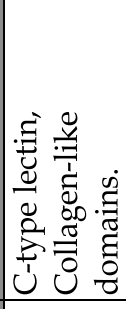 & 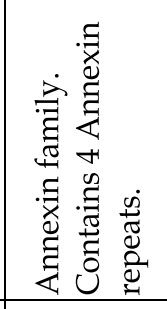 & 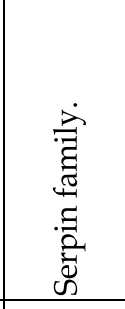 & 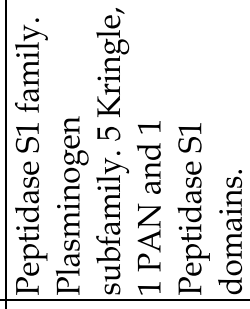 & 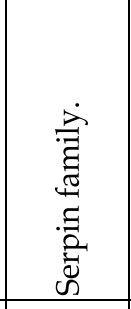 & 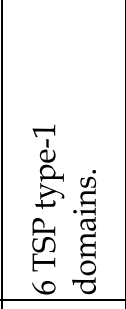 & 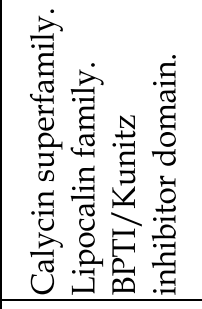 \\
\hline 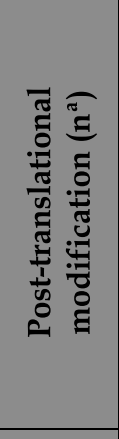 & 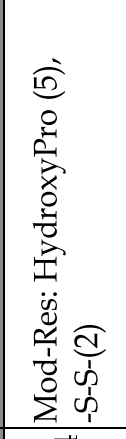 & 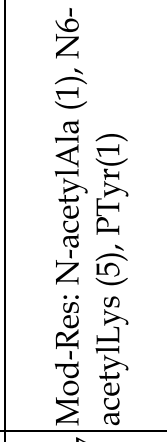 & 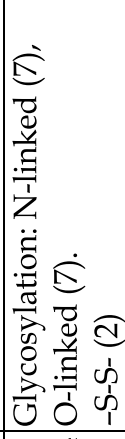 & 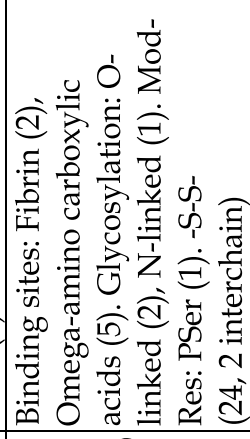 & 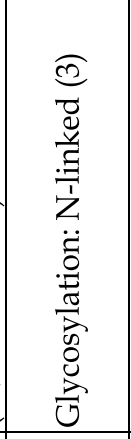 & 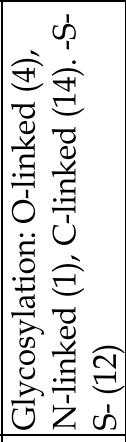 & 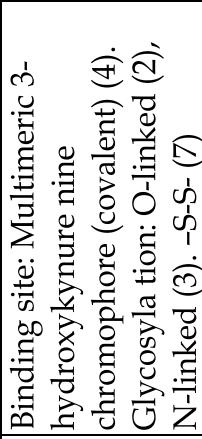 \\
\hline 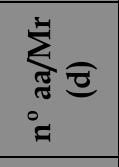 & 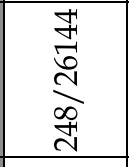 & 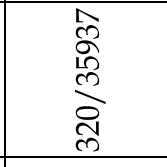 & $\begin{array}{l}10 \\
10 \\
10 \\
10 \\
8 \\
8 \\
10 \\
10\end{array}$ & 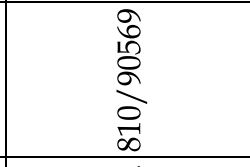 & 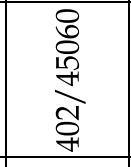 & 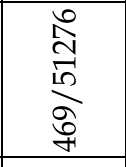 & 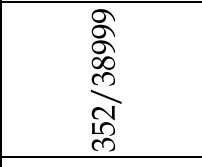 \\
\hline 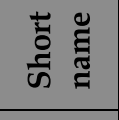 & $\begin{array}{l}u \\
\rho^{\prime} \\
\sum\end{array}$ & 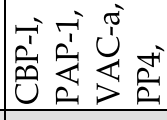 & $\begin{array}{l}E \\
\Xi \\
\end{array}$ & 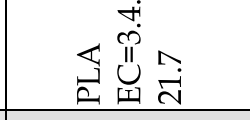 & 昰空 & $\vec{U}$ & 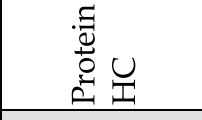 \\
\hline 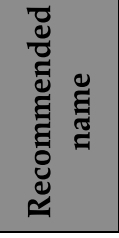 & 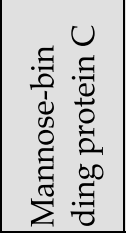 & 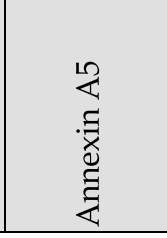 & 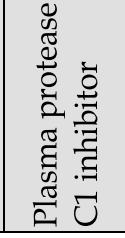 & 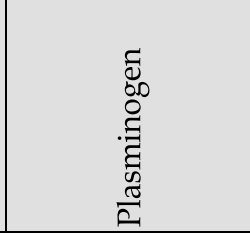 & 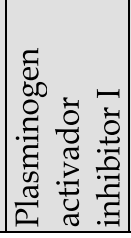 & 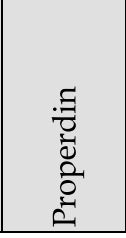 & 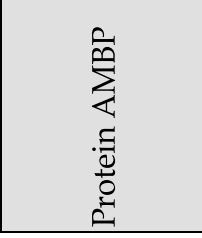 \\
\hline
\end{tabular}




\begin{tabular}{|c|c|c|c|c|c|}
\hline .0ّ & 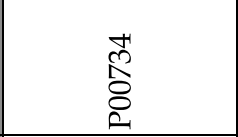 & $\begin{array}{l}\text { ڤొ } \\
\text { ลิ } \\
\text { ¿ }\end{array}$ & $\begin{array}{l}\text { o } \\
\text { ⿵ิ } \\
\text { ¿ } \\
\text { ¿ }\end{array}$ & 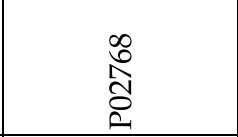 & 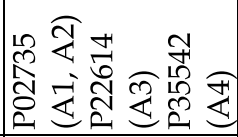 \\
\hline 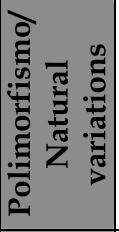 & 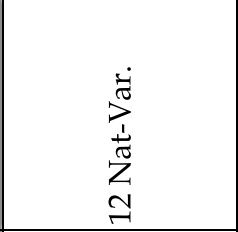 & 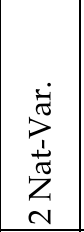 & 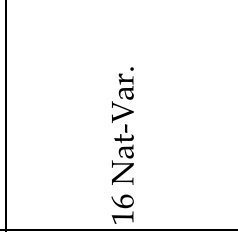 & 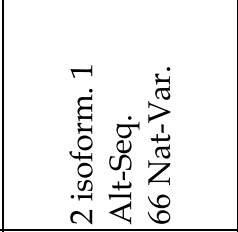 & 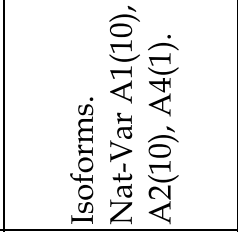 \\
\hline 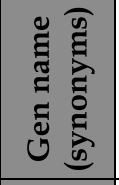 & II & $\stackrel{+}{c}$ & 岁 & 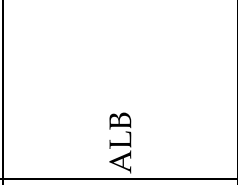 & 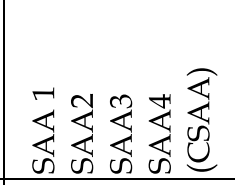 \\
\hline 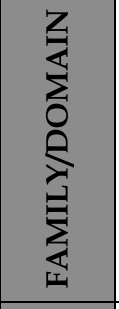 & 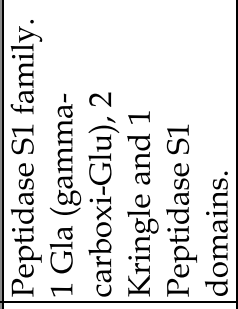 & 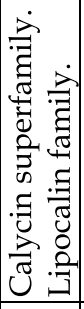 & 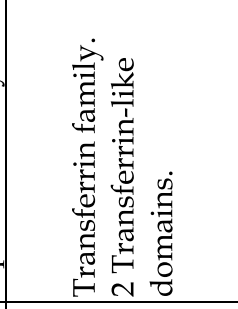 & 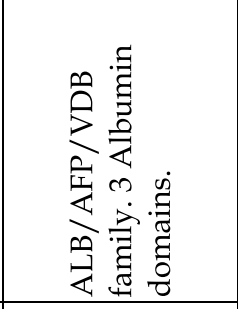 & 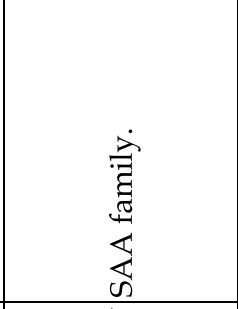 \\
\hline 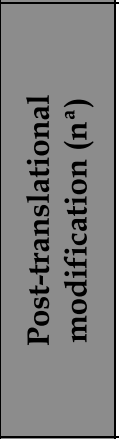 & 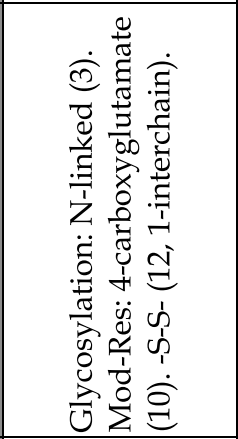 & 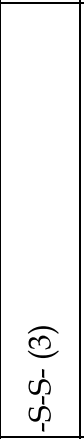 & 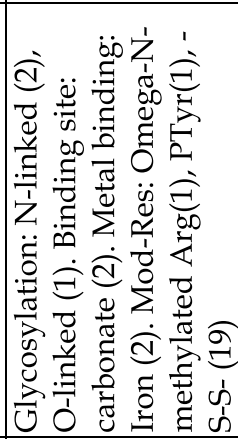 & 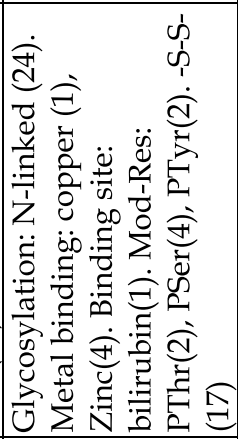 & 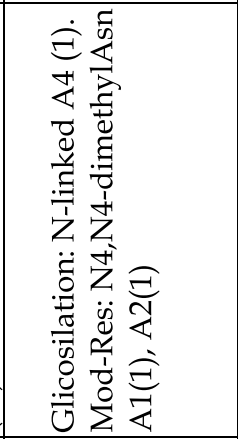 \\
\hline 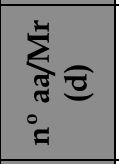 & 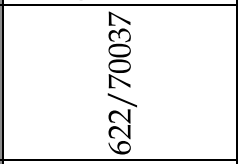 & 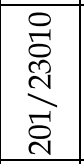 & 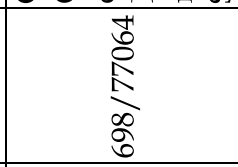 & 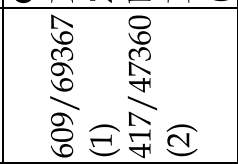 & 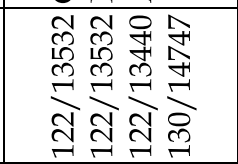 \\
\hline 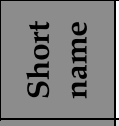 & 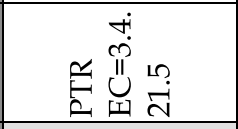 & 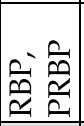 & 离 & $\stackrel{n}{<}$ & 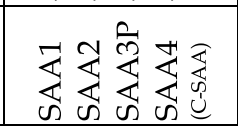 \\
\hline 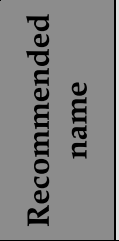 & 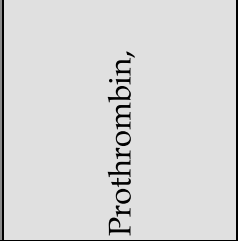 & 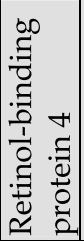 & 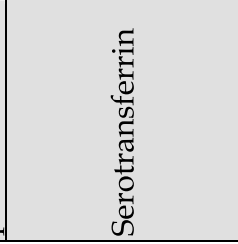 & 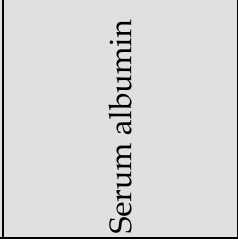 & 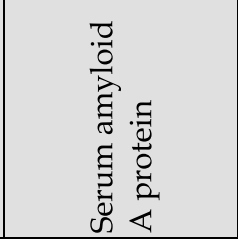 \\
\hline
\end{tabular}




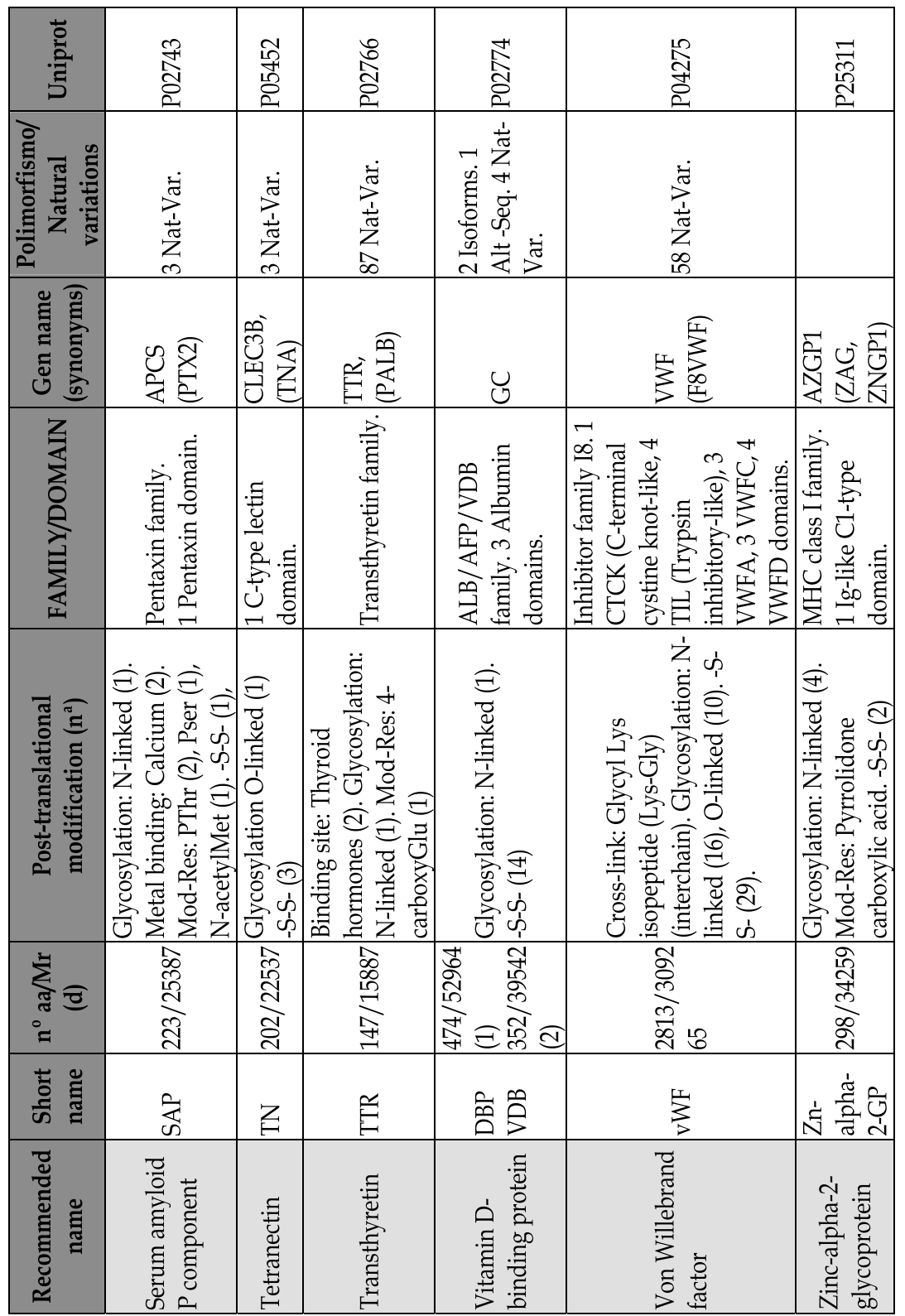

Table 1. Main characteristics of APPs. There are represented the classical APPs and other proteins that have been reported as inflammation related proteins, and cited as putative APPs. Abbreviatures: Alternative Sequence, Alt-Seq; Disulfide Bond, -S-S-; Immunoglobulin, Ig; Interleukin, IL; Major Histocompatibility Complex, MHC; Modified residue, Mod-Res; Natural variants, Nat-Var; Phosphoserine, PSer; Phosphothreonine, PThr; Phosphotyrosine, PTyr; other abbreviatures are specified in the text. 


\begin{tabular}{|c|c|c|c|c|c|c|c|c|c|}
\hline & 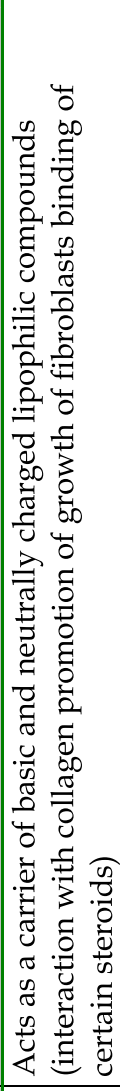 & 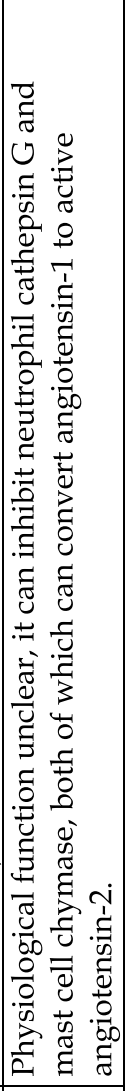 & 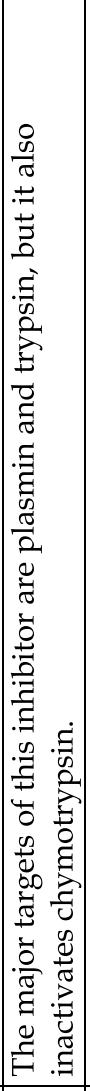 & 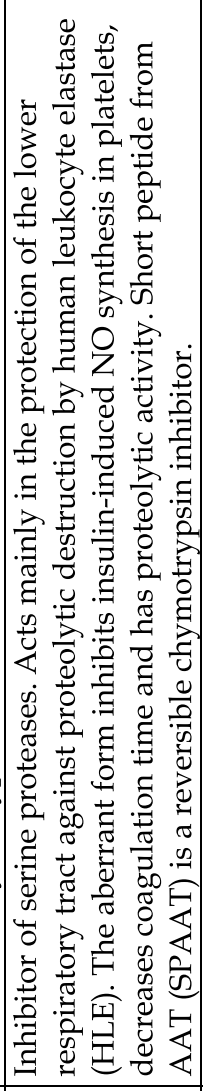 & 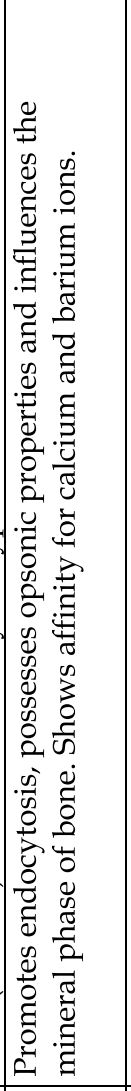 & 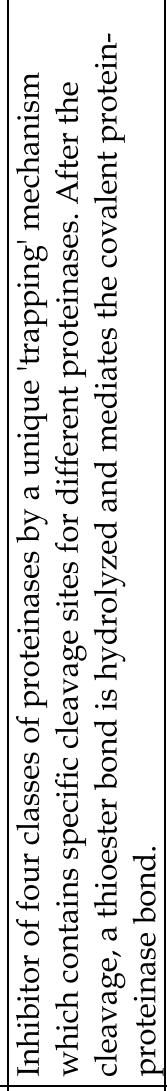 & 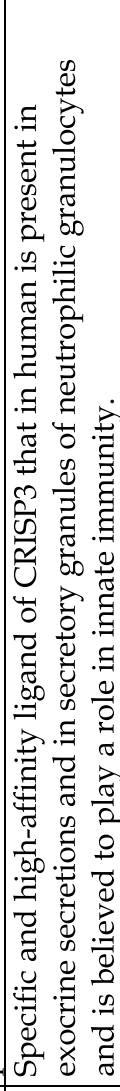 & 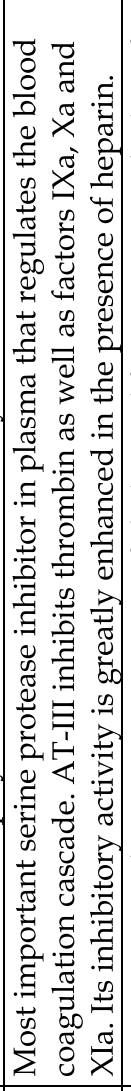 & 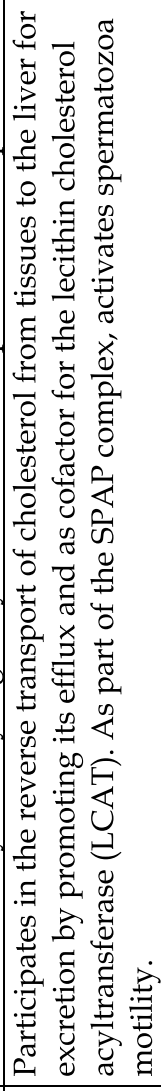 \\
\hline 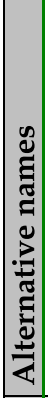 & $\begin{array}{l}\text { T. } \\
0 \\
0 \\
0 \\
0 \\
0 \\
0 \\
0 \\
0 \\
0 \\
0\end{array}$ & 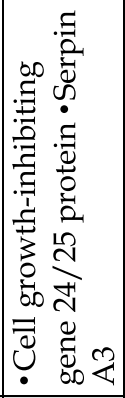 & 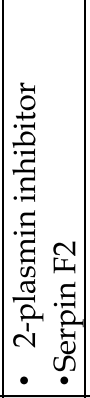 & 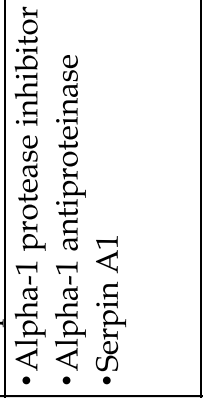 & 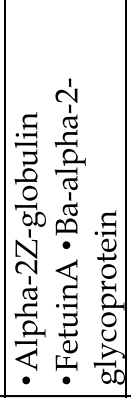 & 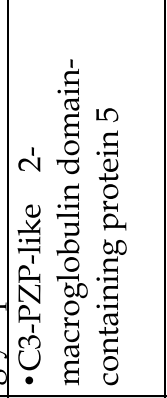 & 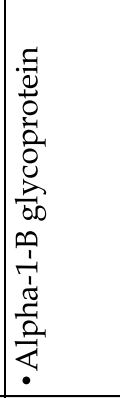 & 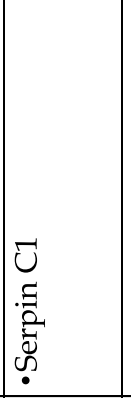 & 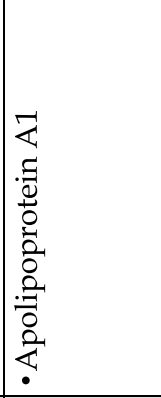 \\
\hline \pm & 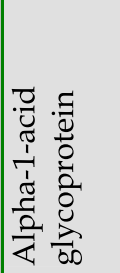 & 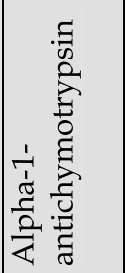 & 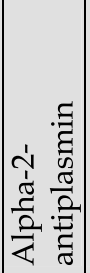 & 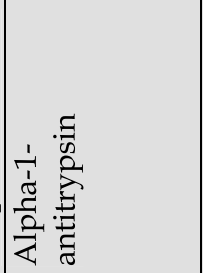 & 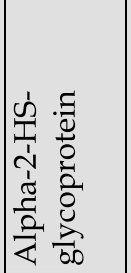 & 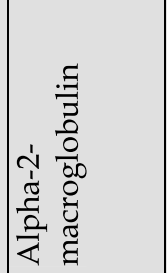 & 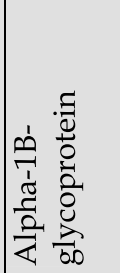 & 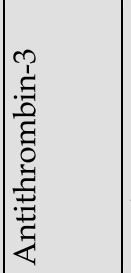 & 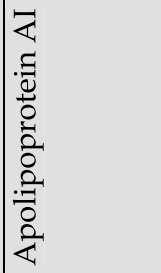 \\
\hline
\end{tabular}




\begin{tabular}{|c|c|c|c|c|c|c|c|c|c|}
\hline 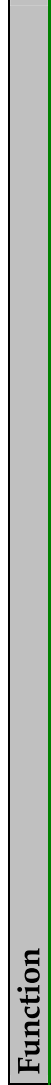 & 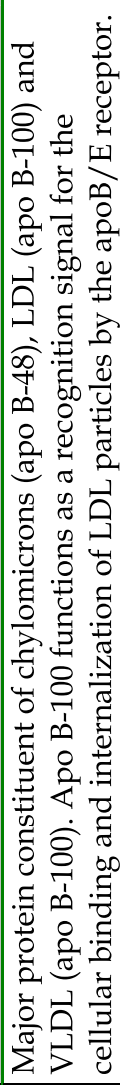 & 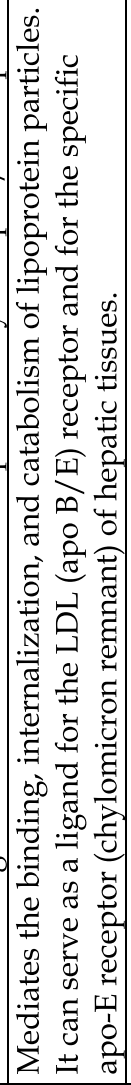 & 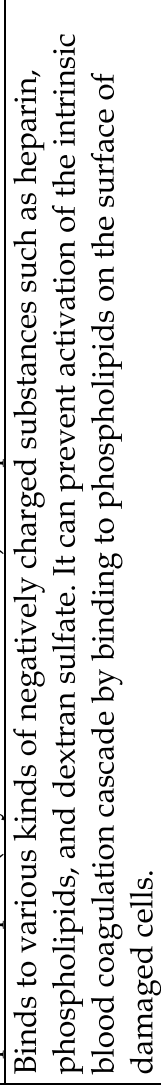 & 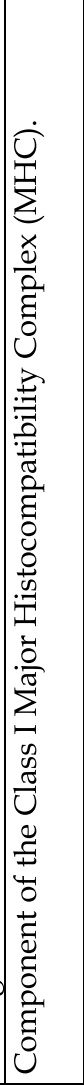 & 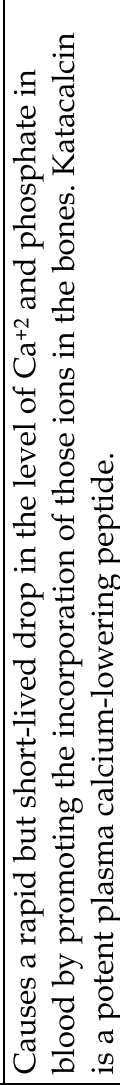 & 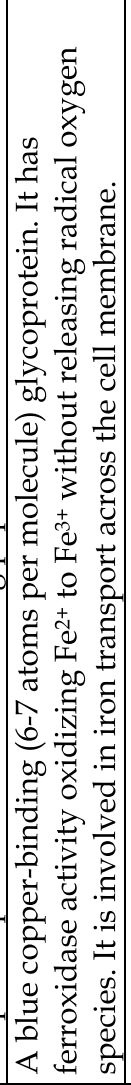 & 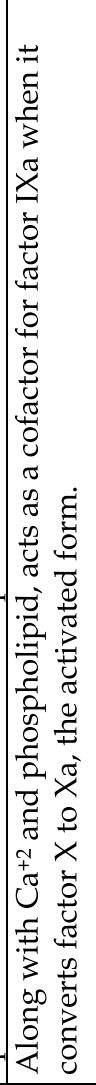 & 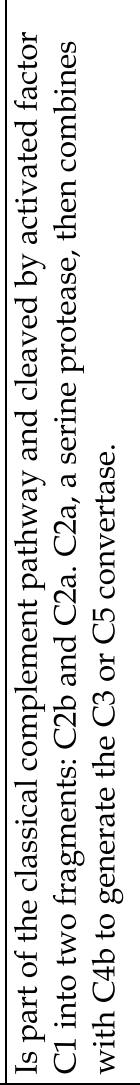 & 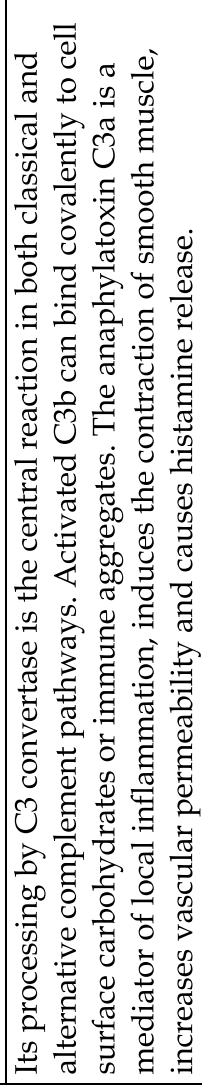 \\
\hline 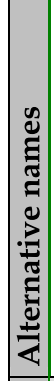 & & & 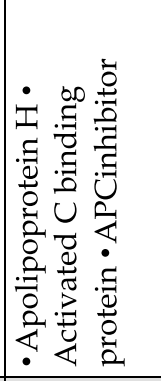 & & 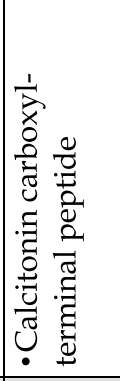 & 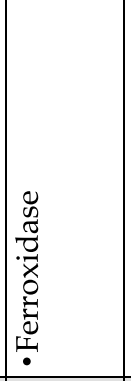 & 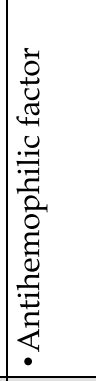 & 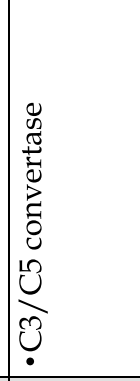 & 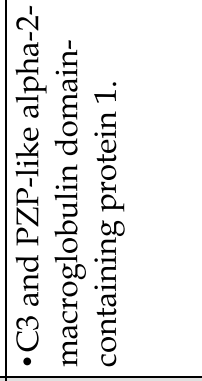 \\
\hline$\stackrel{0}{0}$ & 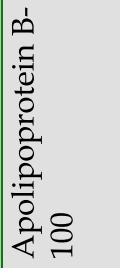 & 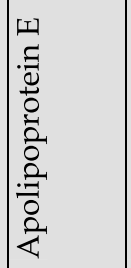 & 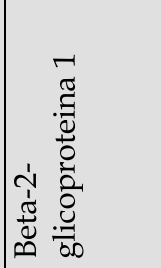 & 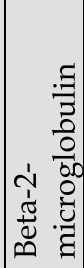 & 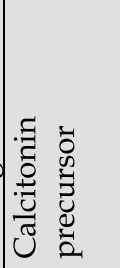 & 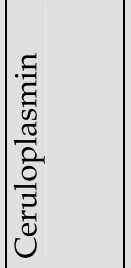 & 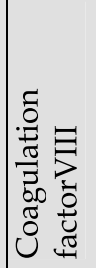 & 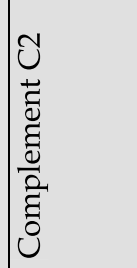 & 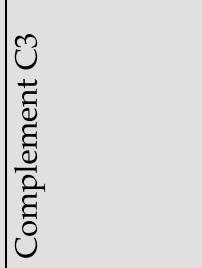 \\
\hline
\end{tabular}




\begin{tabular}{|c|c|c|c|c|c|c|c|c|}
\hline & 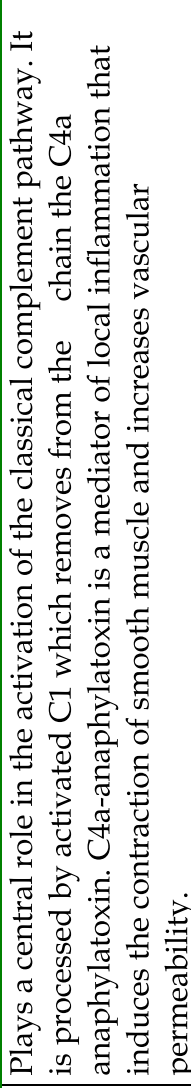 & 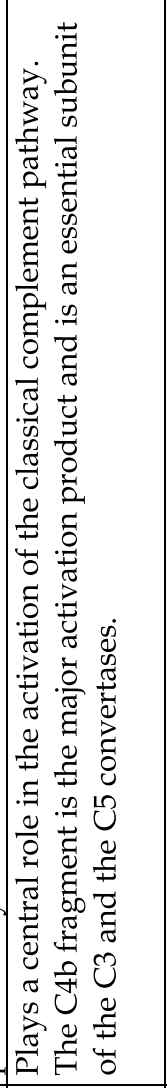 & 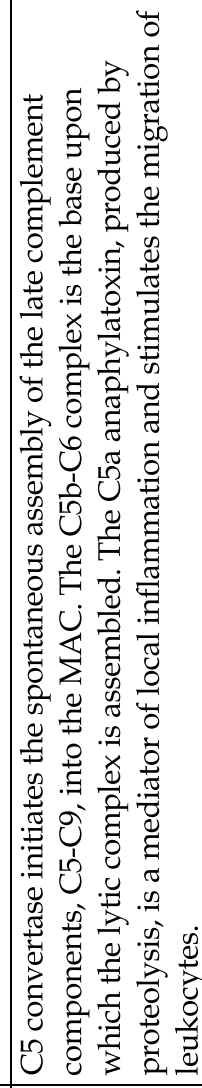 & 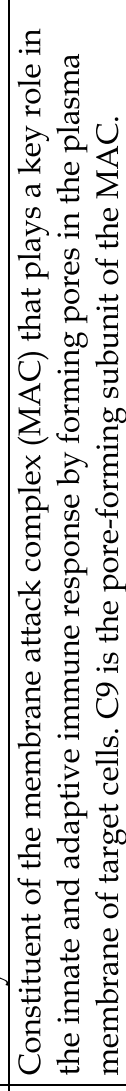 & 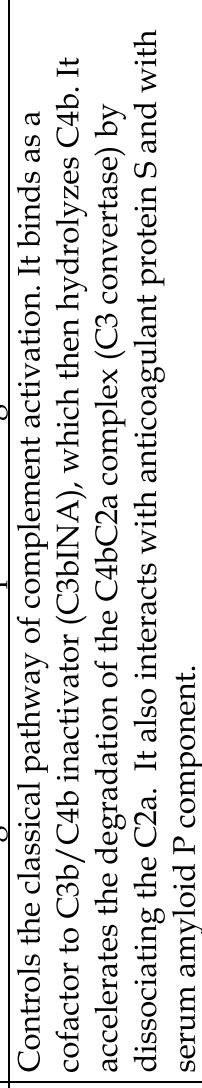 & 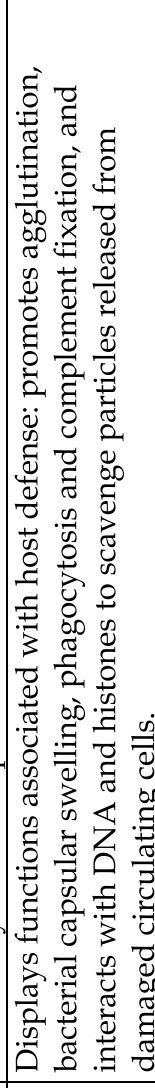 & 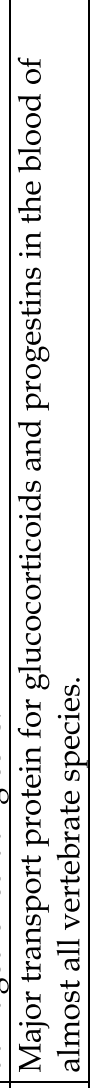 & 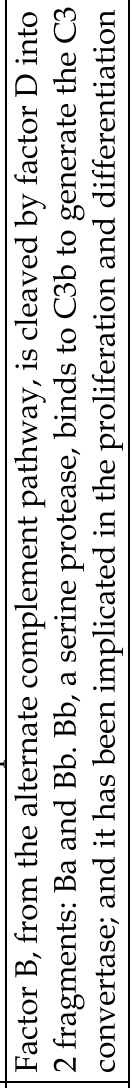 \\
\hline & 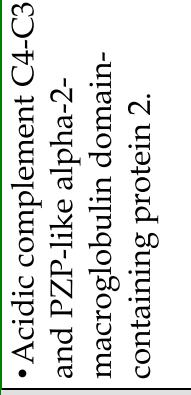 & 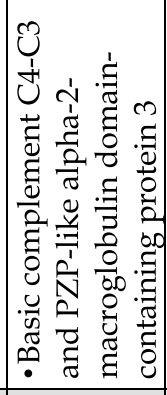 & 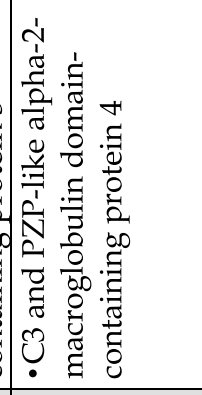 & & 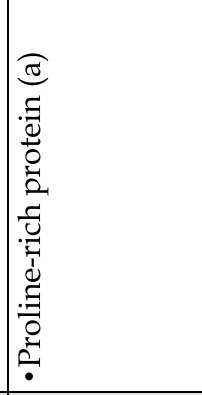 & & 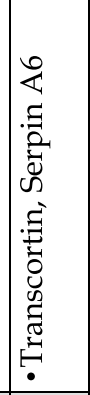 & 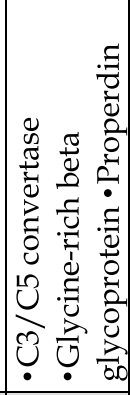 \\
\hline & ç & 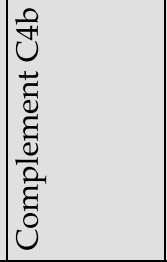 & 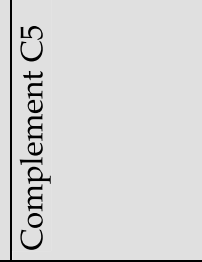 & 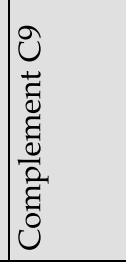 & 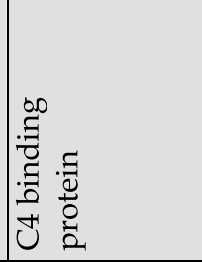 & $\begin{array}{l}0 \\
\vdots \\
0 \\
0 \\
. \geq\end{array}$ & 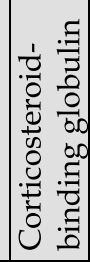 & 0 \\
\hline
\end{tabular}




\begin{tabular}{|c|c|c|c|c|c|c|c|c|c|}
\hline 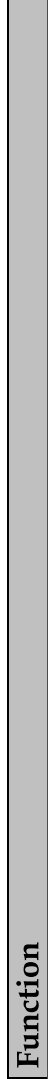 & 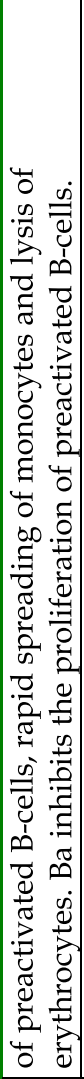 & 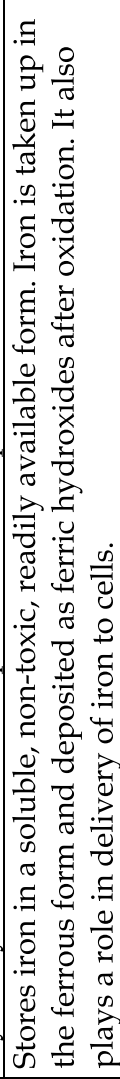 & 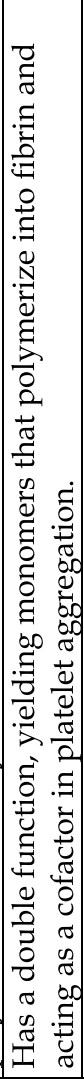 & 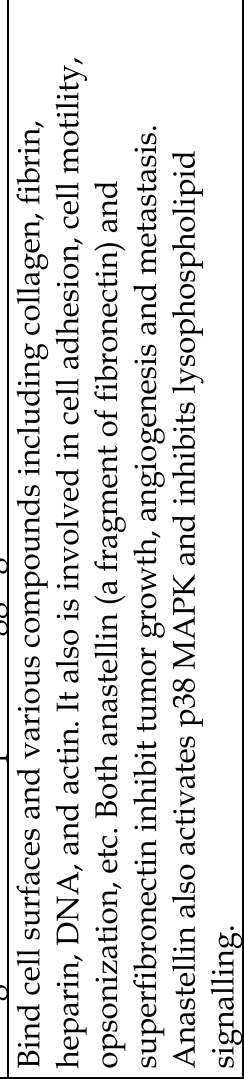 & 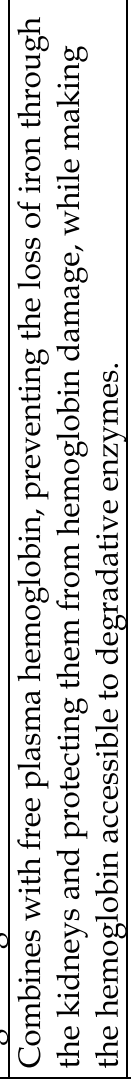 & 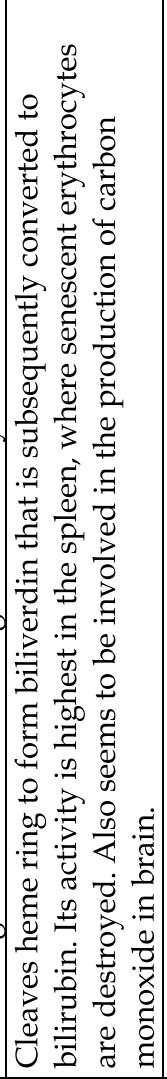 & 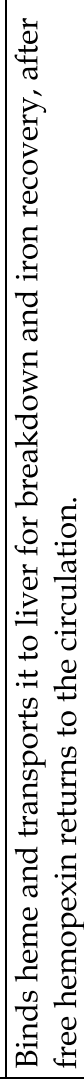 & 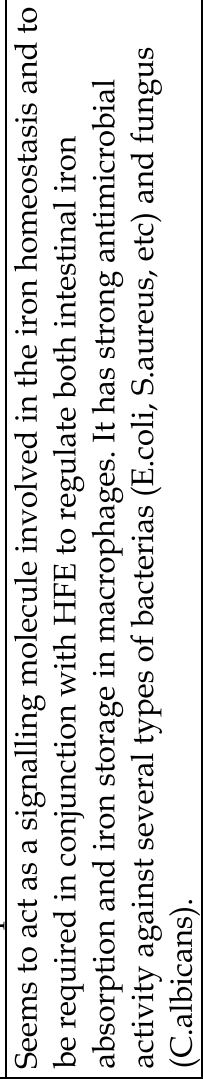 & 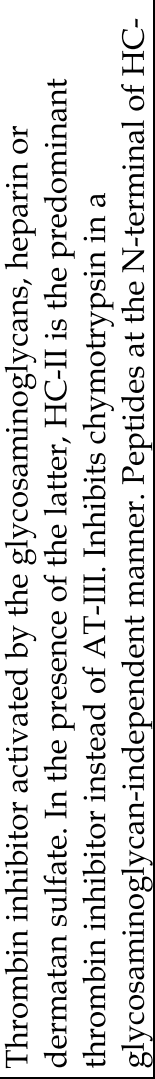 \\
\hline 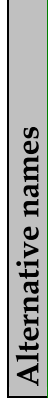 & 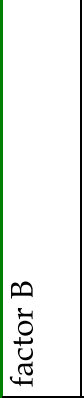 & & & $\begin{array}{l}\frac{1}{3} \\
\frac{1}{0} \\
\frac{0}{60} \\
0 \\
01 \\
\frac{1}{0} \\
0 \\
. \frac{1}{1} \\
\frac{1}{0} \\
0 \\
0\end{array}$ & & & 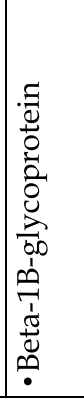 & 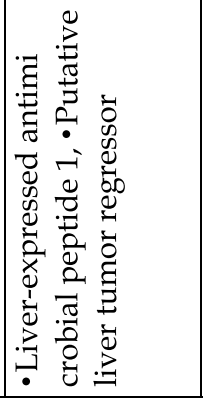 & 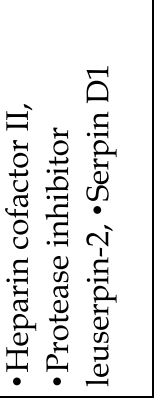 \\
\hline & & 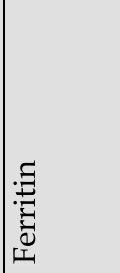 & 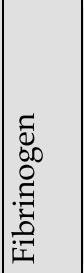 & 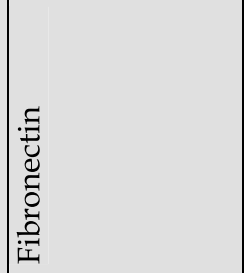 & 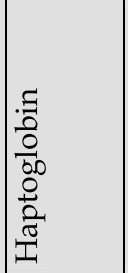 & 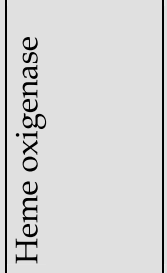 & 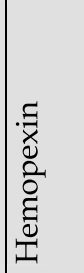 & 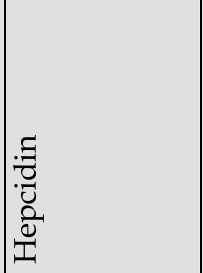 & 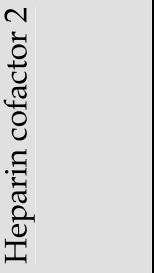 \\
\hline
\end{tabular}




\begin{tabular}{|c|c|c|c|c|c|c|c|c|}
\hline 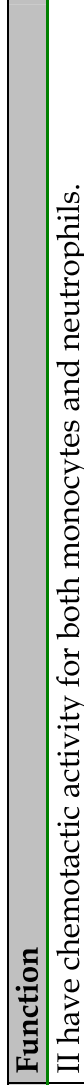 & 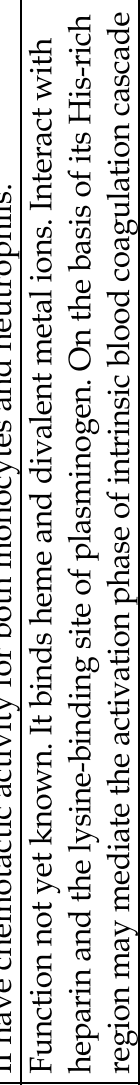 & 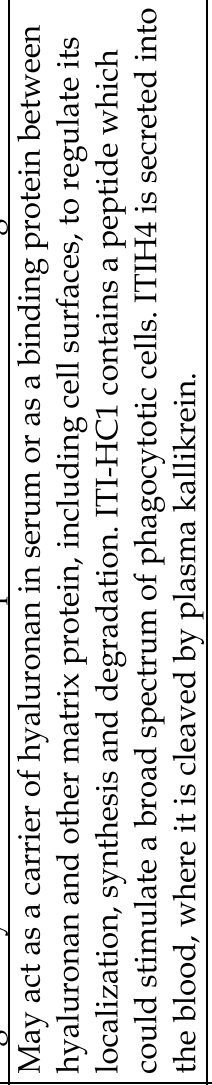 & 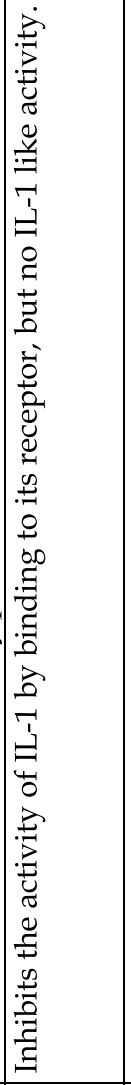 & 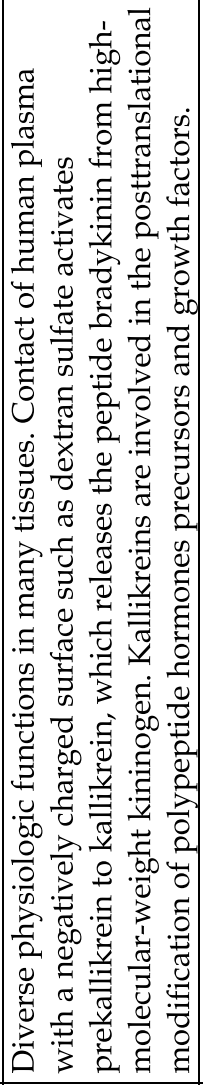 & 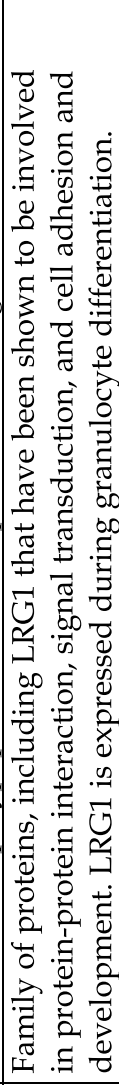 & 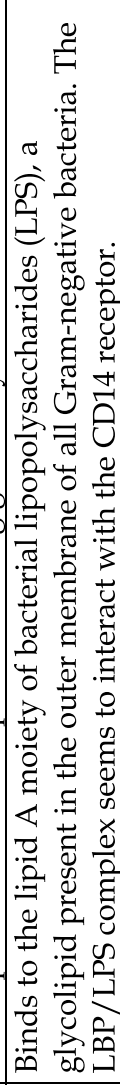 & 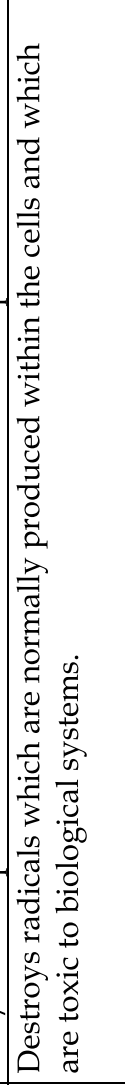 & 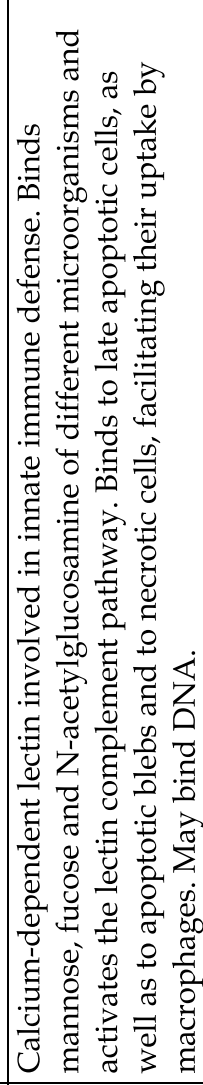 \\
\hline 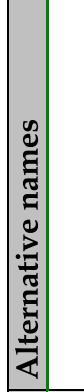 & 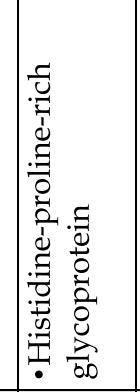 & 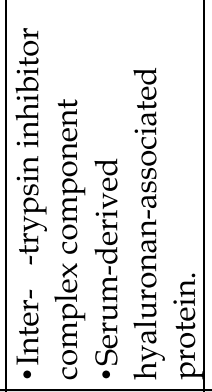 & 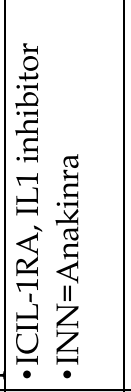 & 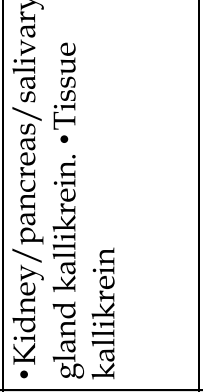 & & 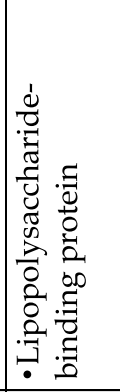 & & 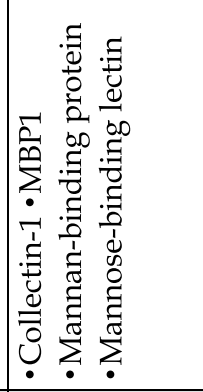 \\
\hline 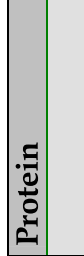 & 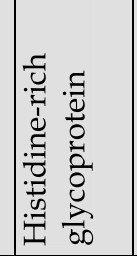 & 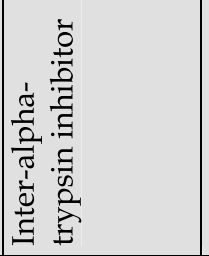 & 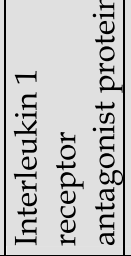 & & 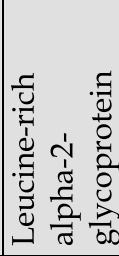 & 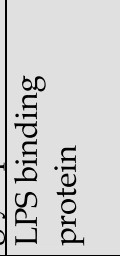 & 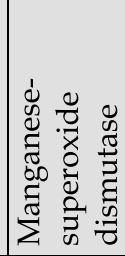 & 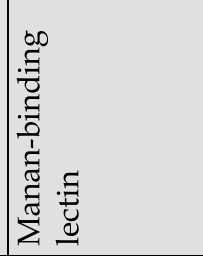 \\
\hline
\end{tabular}




\begin{tabular}{|c|c|c|c|c|c|c|c|c|}
\hline & 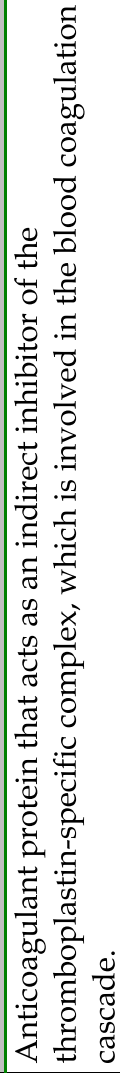 & 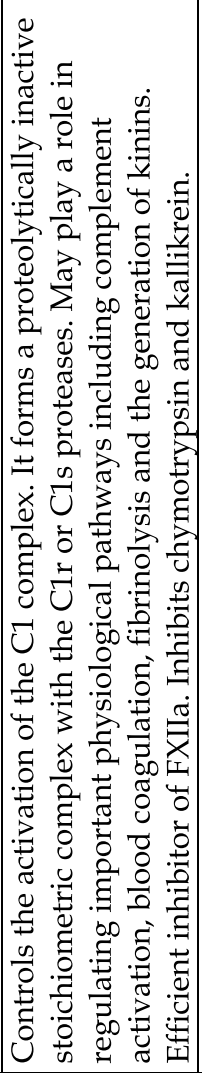 & 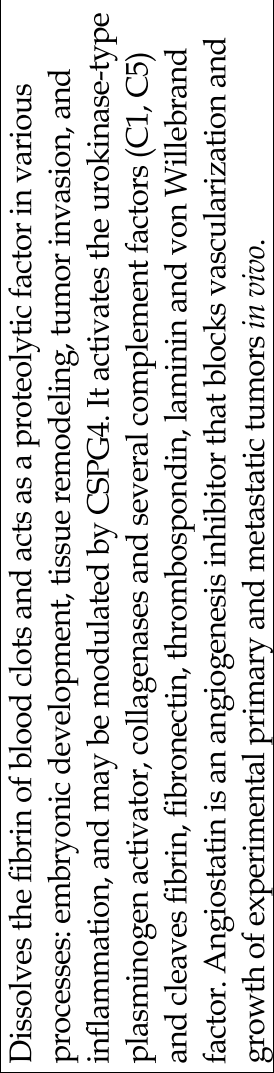 & 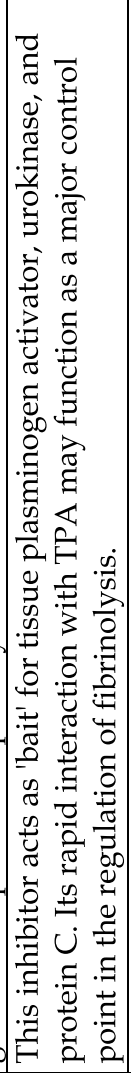 & 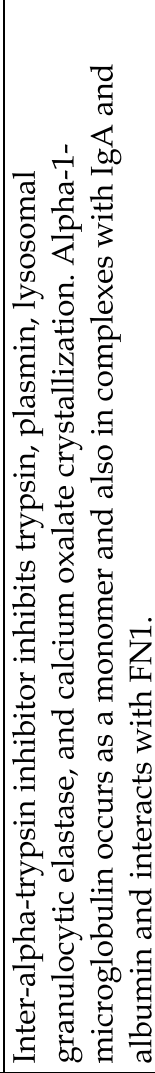 & 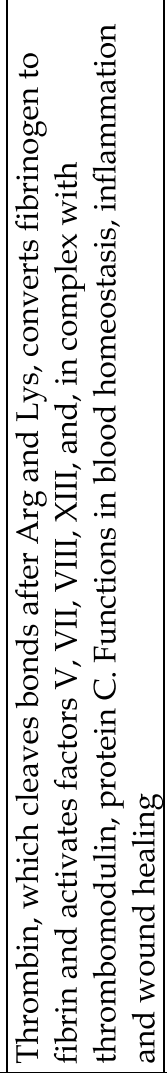 & 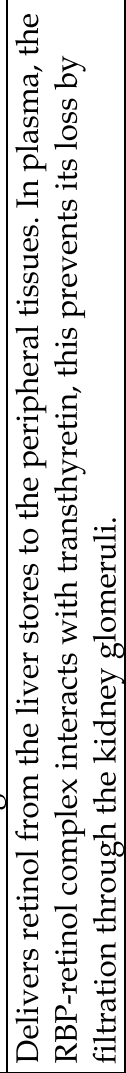 & 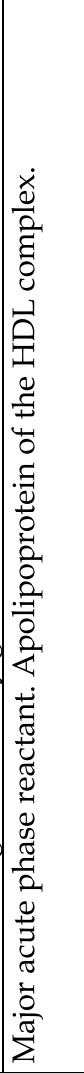 \\
\hline 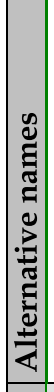 & 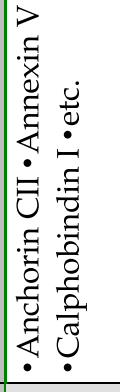 & 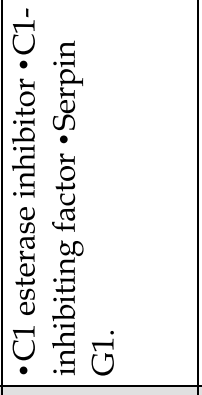 & 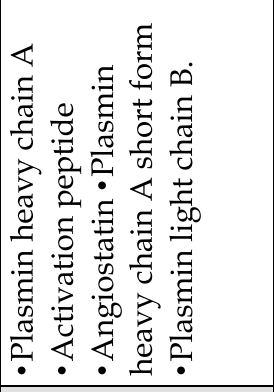 & 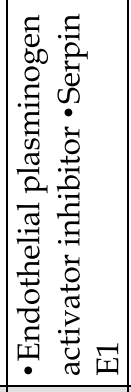 & 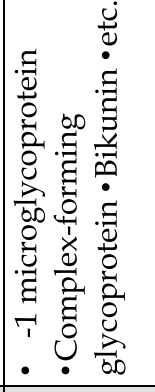 & 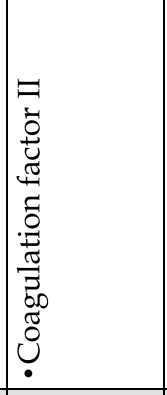 & 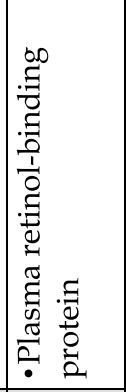 & 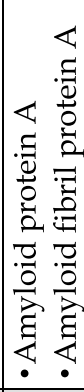 \\
\hline $\begin{array}{r}0 \\
0 \\
0 \\
0 \\
0 \\
0\end{array}$ & $\approx$ & 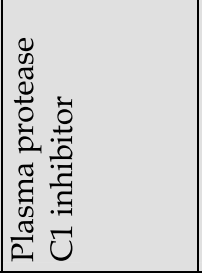 & 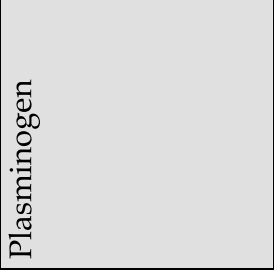 & 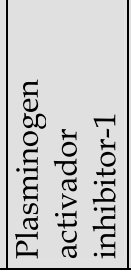 & 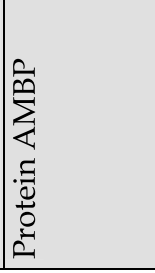 & $\begin{array}{l}: 0 \\
0 \\
0 \\
0 \\
0 \\
0 \\
0 \\
0 \\
\end{array}$ & 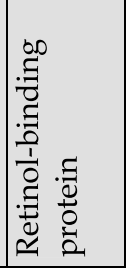 & 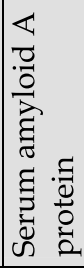 \\
\hline
\end{tabular}




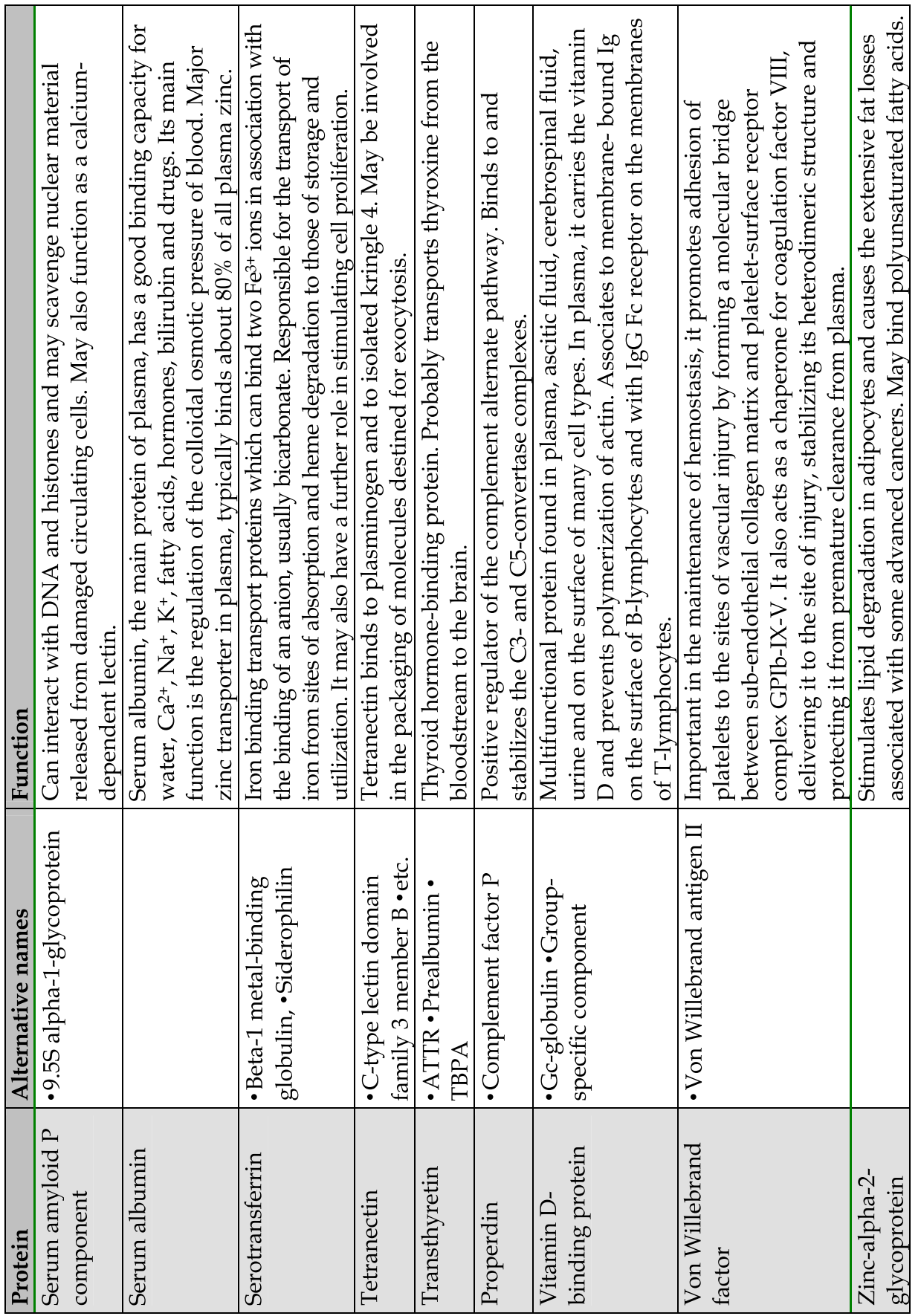

Table 2. Main functions of Acute Phase Proteins. 
Existing APPs test provide a spectrum of clinical information, including definitive diagnosis of acute events (e.g. CRP), prediction of disease risk (CRP increases in coronary disease) or detection of disease recurrence. Thus, if we check the clinical diagnostic catalog at the Beckman Coulter home page, one of the companies with the broader list of IVD tests for APPs (Table 3), we will find that those tests have the following intended uses https://www.beckmancoulter.com/eCatalog/Catalog/Disease_Management): albumin, to evaluate the hepatic/renal function, infectious diseases, inflammatory responses or nutritional assesment; alpha 1 acid glycoprotein, to assess the existence of infectious diseases or inflammation; alpha 1 antitrypsin, to check the presence of infectious diseases or inflammation; alpha 2 macroglobulin and antithrombin III, to detect anemia, thrombofilia or to assay the hepatic function; ApoA1 and $\mathrm{ApoB}$, to measure the cardiovascular risk; ceruloplasmin, for the evaluation of the hepatic function or the detection of infection/inflammation; complement factors (C3 and $\mathrm{C} 4)$, to evaluate the liver function or unmask autoimmune or inflammatory processes; fibrinogen, for thrombofilia and bledding disorders detection; haptoglobin, to uncover infectious diseases, inflammation, and anemia; plasminogen, to assess fibrinolysis; transferrin, to value the presence of anemia, the nutritional status, the presence of infectious diseases, or the renal function; and finally transthyretin/prealbumin, for hepatic function and nutritional assesment. In addition, detection of beta 2 microglobulin (e.g. Quantikine ${ }^{\circledR}$ IVD ${ }^{\circledR}$ Human $\beta_{2}$ M Immunoassay, $R \& D$ ) is an aid in the diagnosis of autoimmune diseases (rheumatoid arthritis, systemic lupus erithematosus), viral infections or reduced glomerular filtration rates (kidney diseases). On the other hand, low levels of plasminogen activator inhibitor 1 (PAI-1) are linked to bleeding, while its elevation is related with an increased number of blood clots (surgery, infection, diabetes) and elevated risk of heart attack or coronary artery disease (CAD).

\begin{tabular}{lllllll}
\hline & Company name & & & Company name & \\
1 & Abaxis, Inc & ABX & 40 & $\begin{array}{l}\text { Health Chem Diagnostics, } \\
\text { Llc }\end{array}$ & HCD \\
2 & Abbott Laboratories & ABL & 41 & Horiba Abx & HOR \\
3 & Access Bio, Inc & ACC & 42 & $\begin{array}{l}\text { Human Diagnostics } \\
\text { Worldwide }\end{array}$ & HDW \\
4 & $\begin{array}{l}\text { Affinity Biologicals, Inc } \\
\text { Alfa Wassermann }\end{array}$ & AFF & 43 & Ibl, Gmbh & IBL \\
5 & $\begin{array}{l}\text { Diagnostic Technologies, } \\
\text { Inc AWDT }\end{array}$ & 44 & Immunostics, Inc & IMMTICS \\
6 & $\begin{array}{l}\text { American Diagnostica, Inc } \\
7\end{array}$ & AMER & 45 & Immuno, Gmbh & IMM \\
8 & Amico Lab, Inc & AMI & 46 & $\begin{array}{l}\text { Instrumentation Laboratory, } \\
\text { ILAB }\end{array}$ \\
9 & $\begin{array}{l}\text { Arkray, Inc } \\
\text { Arlington Scientific, Inc }\end{array}$ & ARL & 48 & Kamiya Biomedical, Co & KB \\
10 & $\begin{array}{l}\text { Axes-Shield Diagnostics, } \\
\text { Ltd }\end{array}$ & AS & 49 & Kent Laboratories, Inc & KL \\
11 & Bacton Assay Systems & BAS & 50 & Medical Diagnostic & MDT
\end{tabular}




\begin{tabular}{|c|c|c|c|c|c|}
\hline 12 & Baxter Diagnostics, Inc & BAX & 51 & $\begin{array}{l}\text { Technologies } \\
\text { Medical Laboratory } \\
\text { Automation Systems, Inc }\end{array}$ & MLAS \\
\hline 13 & Beckman Coulter, Inc & $\mathrm{BC}$ & 52 & $\begin{array}{l}\text { Nitto Boseki Co., Ltd } \\
\text { (Medical Division) }\end{array}$ & NB \\
\hline 14 & Biocheck, Inc & BCK & 53 & $\begin{array}{l}\text { Novamed, Ltd } \\
\text { Olympus Life Science }\end{array}$ & NVM \\
\hline 15 & Bio/Data, Corp & BIODT & 54 & $\begin{array}{l}\text { Research Europa, Gmbh } \\
\text { (Europa) }\end{array}$ & OLSR \\
\hline 16 & Biokit, S.A. & BK & 55 & $\begin{array}{l}\text { Ortho-Clinical Diagnostics, } \\
\text { Inc }\end{array}$ & OCD \\
\hline 17 & Bio-Medical Products, Corp & BMP & 56 & Polymedco, Inc & POL \\
\hline 18 & Biopool AB & $\mathrm{BAB}$ & 57 & Precisa Intl Corp & PREC \\
\hline 19 & Boditech Dioagnostics, Inc & BODIT & 58 & Quidel Corp & QUID \\
\hline 20 & Brahms AG & BAG & 59 & R2 Diagnostics, Inc & \\
\hline 21 & $\begin{array}{l}\text { Carolina Liquid } \\
\text { Chemistries, Corp }\end{array}$ & CLC & 60 & Randox Laboratories, Ltd & RDXL \\
\hline 22 & Cenogenetics, Corp & CNG & 61 & Reagents Applications, Inc & RA \\
\hline 23 & Cezanne SAS & CSAS & 62 & Remel, Inc & REM \\
\hline 24 & Cliniqa, Corp & CLINQ & 63 & R\&D Systems & R\&D \\
\hline 25 & $\begin{array}{l}\text { Covance Research } \\
\text { Products, Inc }\end{array}$ & CRP & 64 & Roche Diagnostics, Gmbh & ROD \\
\hline 26 & Dade Behring, Inc & DB & 65 & $\begin{array}{l}\text { Rowley Biochemical } \\
\text { Institute, Inc }\end{array}$ & ROW \\
\hline 27 & Dako Denmark A/S & DAK & 66 & $\begin{array}{l}\text { Seradyn, Inc } \\
\text { Shanghai Shenfeng }\end{array}$ & SER \\
\hline 28 & Dexall Biomedical Labs, Inc & DEX & 67 & $\begin{array}{l}\text { Biochemistry Reagent Co, } \\
\text { Ltd }\end{array}$ & SSBR \\
\hline 29 & Diagnostica-Stago & DS & 68 & $\begin{array}{l}\text { Siemens Medical Solutions } \\
\text { Diagnostics }\end{array}$ & SMSD \\
\hline 30 & Diagnostix Technology, Inc & DT & 69 & Stago R\&D & STG \\
\hline 31 & Diamedix Corp & DIAMED & 70 & Sterling Diagnostics, Inc & SD \\
\hline 32 & Diasorin, Inc & DIA & 71 & Teco Diagnostics & TECO \\
\hline 33 & $\begin{array}{l}\text { Diasys Diagnostics } \\
\text { Systems, Gmbh }\end{array}$ & DSYS & 72 & Texas Immunology & TEXAS \\
\hline 34 & Dominion Biologicals, Ltd & DOM & 73 & The Binding Site, Inc & TBS \\
\hline 35 & DRG Instruments, Gmbh & DRGI & 74 & Tosoh Bioscience, Inc & TOS \\
\hline 36 & Eucardio Laboratory, Inc & EUL & 75 & Trinity Biotech, Plc & TB \\
\hline 37 & Fisher Diagnostics & FISH & 76 & $\begin{array}{l}\text { Ventana Medical Systems, } \\
\text { Inc }\end{array}$ & VMS \\
\hline 38 & Genetic Technologies, Inc & GEN & 77 & Wako Pure Chemicals, Inc & WAKO \\
\hline 39 & Globalemed, Llc & GBL & 78 & $\begin{array}{l}\text { YK \& E Advance Trading, } \\
\text { Inc }\end{array}$ & YK\& \\
\hline
\end{tabular}

Table 3. Some companies developing in vitro diagnostics (IVD) tests for clinical use.

This table is not expected to be a comprehensive summary of all existing IVD companies. Mainly, data have been collected from different sources, like the US Food and Drug Administration Home Page (http://www.fda.gov), ZapConnect.com, or the home page of each company. 
Amongst this first group of IVD tests for APPs, C-reactive protein/CRP is, by far, the most frequent offered by different companies (Table 4). That is due to a dual use: On the one hand, a low sensitivity test for inflammation or infectious/autoimmune diseases. On the other hand, systemic inflammation and CRP levels increase as the amount of visceral adipose tissue and the waist circumference become excessive, and that is because CRP (as well as the fibrinolysis inhibitor PAI-1) are not only produced by liver but also by adipocytes (Libby et al., 2010). Based on that, a high-sensitivity assay for cardiovascular risk (denoted hs-CRP) has been developed to predict future clinical cardiovascular events. Elevation of average hs-CRP level is linked to atheroesclerosis, and therefore point to a higher potential of stroke, myocardial infarction or severe peripheral vascular disease development. Thus, apparently healthy people with some inflammation (above median hsCRP) but below median levels of low-density lipoprotein (LDL) could nonetheless benefit from statin therapy (Libby et al., 2010).

Moreover, these companies are continuosly adding new products to their growing portfolio of IVD assays, as it is the case for some acute phase reactants (Table 4). For example, tests for factor VIII (hemophilia A) and von Willebrand (von Willebrand disease) are included within the pannels of reagents to check hemostasis, and alpha 1 microglobulin is been introduced in market for the functional evaluation of liver and kidney, diabetes and stroke. As well, elevation of calcitonin precursor levels in plasma represent a high risk for progression to severe sepsis if the measurement of this acute phase reactant is done shortly after the systemic infection process has started (on the first day). Still, there are other IVD tests that have not been included in Table 4, like properdin Factor B (autoimmune diseases) or apolipoprotein (a)(cardiovascular risk), that are also manufactured by some companies.

A large majority of APPs are typically measured by enzyme immunoassays (EIA, ELISA), but also by immunoturbidimetric or nephelometric technologies through chemistry analyzers such as Alfa Wassermann Alera/ACE, Roche/Hitachi, Roche/Cobas, Beckman Synchron ${ }^{\circledR}$, Beckman/Olympus, Abbot Aeroset, Siemens/Bayer Advia or Siemens/Dade Dimension. There are some radioimmuno- (RIA) (e.g., Abbott Beta-2-Microglobulin RIA) and chromogenic assays too (e.g., BCG method for albumin), and some of these analytes have waived versions (simple and accurate, according to the CLIA complexity classification; e.g., Abaxis, Inc).

\section{New methods and technologies for the quantification of acute phase proteins: APPs as disease biomarkers}

The high-throughput genomic and proteomic technologies, combined with bioinformatics, give the most recent approaches to the study and analysis of APPs in human body fluids (plasma, serum). There are different methods that permit the simultaneous qualitative/quantitative analysis of several APPs from small volume of samples: 2-DE/MS, 2-DE-DIGE/MS, MALDI-TOF-MS, SELDI-TOF-MS, label-free LC-MS profiling, CE-ESI-MS, isotope tagging/MS/MS, or antibody arrays for serum profiling. Proteomic methods can now detect more than 1000 proteins in plasma at the same time. However, the comprehensive analysis of the proteome of any body fluid is still beyond our reach, despite great methodological advances in recent years. Several major challenges must be faced by researchers. For instance, proteomics of body fluids is limited to a $\sim \mu \mathrm{g} / \mathrm{L}(\mathrm{ng} / \mathrm{mL})$ 


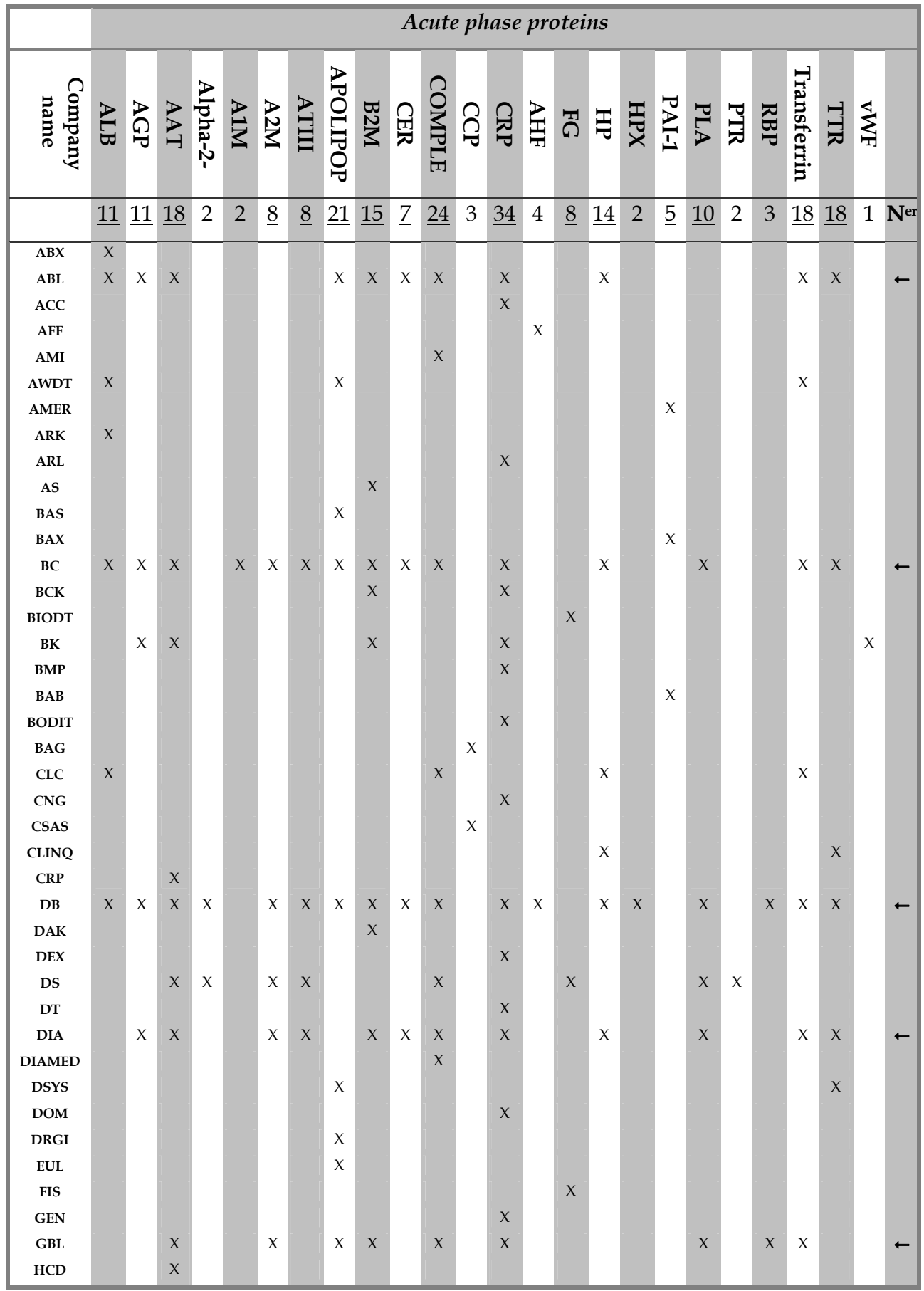




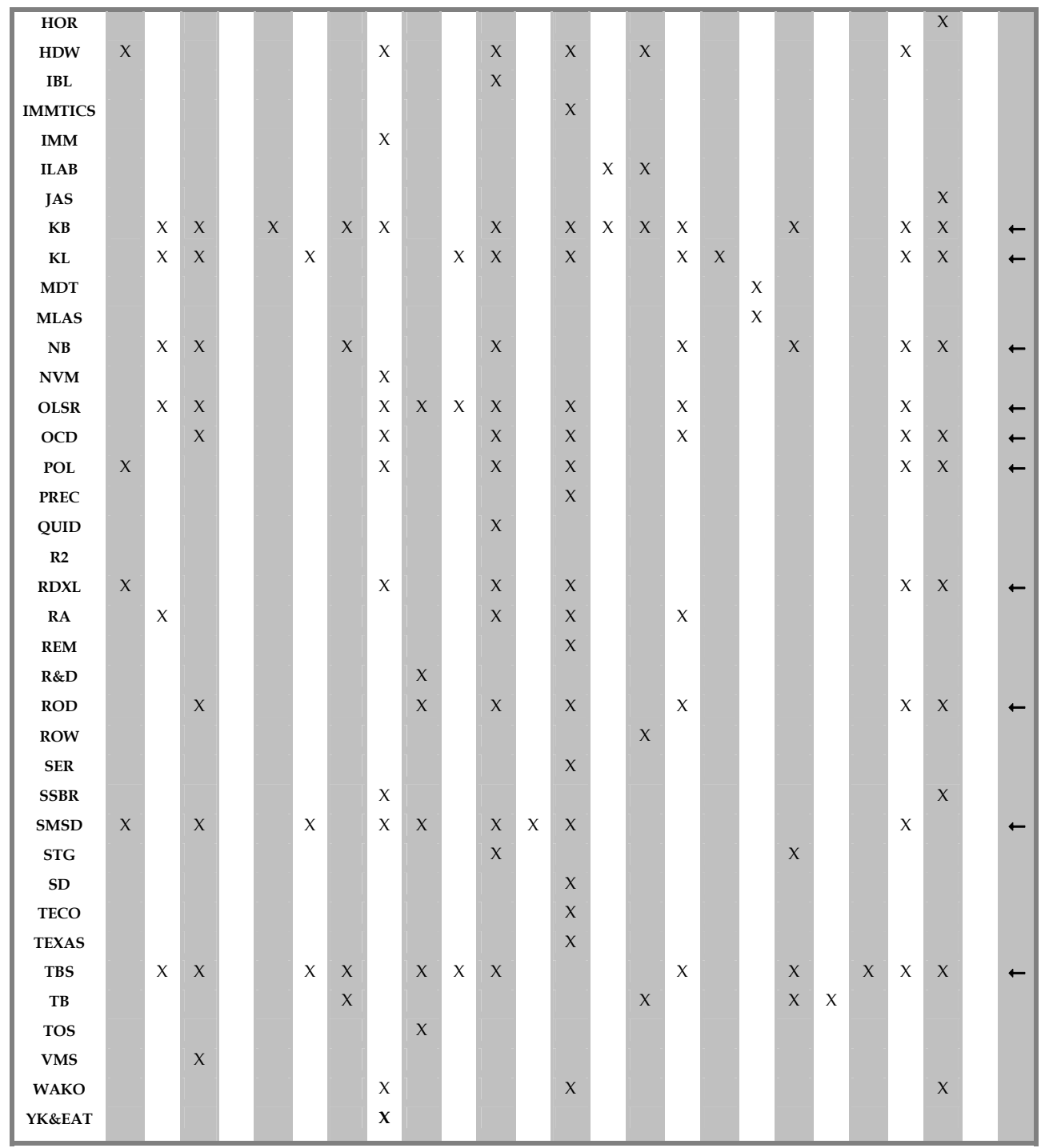

Table 4. In vitro diagnostic (IVD) tests for the quantitative determination of acute phase proteins in serum or plasma.

This table is not intended to be a comprehensive summary. Data come from different sources, like the US Food and Drug Administration Home Page (http://www.fda.gov), ZapConnect.com, or the home page of each company. Sometimes this one does not offer directly IVD tests for some APPs, but can do it through strategic alliances that are not reflected in Table 3 (e.g., Beckman Coulter and Instrumentation Laboratory and the HemosIL® Fibrinogen- $C$ kit). Abbreviations used for the name of the companies are the same as in Table 3. Abbreviations for APPs are the same as in Table 1. 
sensitivity, which is roughly 1000 -fold less sensitive than the most specific immunoassays. Another additional problem is the range of protein concentrations in body fluids. The dynamic range of plasma proteins concentrations spams from that much as $40 \mathrm{mg} / \mathrm{mL}$ for albumin ( $50 \%$ of the total mass of plasma proteins) to less than $4 \mathrm{pg} / \mathrm{mL}$; i.e., the concentration of many cytokines. Therefore, we are talking about 10-12 orders of magnitude, which means that disease-mediated alterations in soluble mediators (e.g., cytokines), or modifications in those released into the circulation through cell-leaking processes during normal cell turnover (apoptosis) or specific injuries (e.g., myocardial infarction), are hardly detectable. For example, the most common technology for fractionating and identifying proteins, 2-DE, has a range of detection of no more than 3-4 orders of magnitude with differential gel electrophoresis (DIGE). About $99 \%$ of total protein mass in plasma depends on only 22 abundant species, like albumin (35-45 mg/mL), IgG, IgA, IgM (12-18 mg/mL), $\alpha 2$ and $\beta$-lipoproteins (LDL; 4-7 mg/mL), fibrinogen $(2-6 \mathrm{mg} / \mathrm{mL}), \alpha 1$-antitrypsin $(2-5$ $\mathrm{mg} / \mathrm{mL}), \alpha 2$-macroglobulin $(2-4 \mathrm{mg} / \mathrm{mL})$, transferrin $(2-3 \mathrm{mg} / \mathrm{mL}), \alpha 1$-acid-glycoprotein $(1$ $\mathrm{mg} / \mathrm{mL})$, hemopexin $(1 \mathrm{mg} / \mathrm{mL})$, $\alpha$-lipoproteins (HDL, 0.6-1.5 mg/mL), haptoglobin $(0.3-2$ $\mathrm{mg} / \mathrm{mL})$, prealbumin $(0.3-0.4 \mathrm{mg} / \mathrm{mL})$ or ceruloplasmin $(0.3 \mathrm{mg} / \mathrm{ml})$. Most of them are acute phase reactants; i.e., plasma resident proteins with a molecular mass larger than the kidney filtration cutoff and large half-lives, both characteristics ensuring an extended residence time and high protein concentrations $(45 \mathrm{mg} / \mathrm{mL}-0.001 \mathrm{mg} / \mathrm{mL})$. Thus, this high abundance makes them easily accessible to proteomic technologies, although there are still a couple of questions to consider: complexity of this family of proteins and their timedependent concentration modulation (kinetics).

Regarding complexity, the most important APPs are usually glycoproteins. This means that APPs, like immunoglobulins, are highly heterogeneous (Anderson and Anderson, 2002). The difficulty of their analysis is even enhanced by processing events, that lead to the generation of smaller proteins or peptides from larger precursors (both the plasma and cells contains numerous proteases), or the presence of genetic variants in acute phase reactants as apoE, apoH, transferrin or haptoglobin. Two-dimensional electrophoresis (2-DE) was the first used method in clinical biofluids proteomics, and still is one of the most used nowadays for different reasons. After the introduction of high resolution 2-DE in 1975 this technique was applied to the plasma proteins (Anderson \& Anderson, 1977). These authors were able to resolve by $2-\mathrm{DE}$ about 300 or more spots and a number of about 40 distinct plasma proteins. This initial amount has been extended to 626 identified spots, 1966 detected spots and 69 proteins in the current Swiss 2D-Page Web site (http://expasy.org/swiss-2dpage), which may represent the limit in terms of 2-DE analysis of unfractionated plasma. Therefore, it was clear very soon than 2-DE had limitations with respect to the resolution to cope with the extraordinary dynamic range of plasma proteins concentrations and the complexity issue, apart from additional problems: low and high molecular weight, hydrophobic, and very acid or basic proteins. Still, isolectric points of APPs are mostly between $\mathrm{pH} 5$ and 6, and these proteins are originated mainly from liver secretion (not cell leakage; i.e., hydrophilic) and have a medium-high molecular size. In addition, there are means to reduce sample complexity and enhance the loading capacity of 2-DE (see below). Therefore, 2-DE is a technique specially suited to screen the acute phase response, and can be even essential to determine the post-translational and genetic modifications of APPs.

Nevertheless, and largely because of novel methods of serum fractionation and MS based protein identification, after the turn of the century the number of plasma proteins that could be identified increased over time. Thus, thanks to extensive fractionation serum 
fractionation and tandem mass spectrometry, Adkins et al. (2002) were able to identify 490 proteins. That number suffered a huge increase in 2004, to achieve an identification of 1,175 non-redundant plasma proteins, by using multidimensional chromatography and MS analysis (Anderson et al. 2004). At present, the Human Proteome Organization (HUPO) Plasma Proteome Project (PPP) has a list of 3020 plasma proteins (http://www.hupo.org/ research/hppp/)

\subsection{Quantitative proteomics technologies applied to the study of APPs}

However, the elaboration of a detailed list of serum/plasma proteins provides relatively limited biological information. To understand the biology of any pathological process, quantitative analysis of these proteins is essential. Perhaps, 2-DE or 2-DE fluorescence differential gel electrophoresis (DIGE), followed by MS analysis, remains as the most commonly used method (see Table 5). DIGE is a quantitative technique that enhance the dynamic range of 2-DE up to four orders of magnitude. DIGE is commercially available from GE Healthcare, and allows the direct comparison of different samples at the same time on a single 2-DE gel. This technique involves the use of up to three different samples and three cyanide dyes (Cy2, Cy3, and Cy5). These dyes have an NHS-ester reactive group designed to covalently attach to the epsilon amino group of lysine of proteins via an amide linkage. In addition, dyes label 1-2\% of all the available proteins (minimal labelling, about one dye molecule per protein), without changing their $\mathrm{pI}$ and adding only $0.5 \mathrm{kDa}$ to the protein mass. Normally, a pooled sample, comprising equal amounts of each of the initial samples within the study, is labelled with Cy2 and used as internal control. This internal standard allows normalization and is an effective way to increase accuracy and reproducibility during quantification. On the contrary, one particular disadvantage of DIGE is the equipment required for visualization and spot excision, and another major problem with these 2-DE based methodologies is that they are labor intensive and difficult to automate. In addition, as we have just commented, they are not able to effectively mine the low abundance biofluids proteome $(<100 \mathrm{ng} / \mathrm{ml})$. Therefore, the detected proteins limit primarily to high $(0.1-40 \mathrm{mg} / \mathrm{ml})$ and medium abundance $(0.1-100 \mu \mathrm{g} / \mathrm{ml})$ species, which is enough to study the classical acute phase reactants (e.g., CRP $<10 \mu \mathrm{g} / \mathrm{ml}$ ), but does not allow to find new ones. All these limitations of 2-DE based methods are driving the development of new proteomic methodologies for serum/plasma profiling that seek to reduce the workload of 2-DE (since they use automated equipment), such as MALDI-TOFMS, SELDI-TOF-MS, label-free LC-MS profiling, CE-ESI-MS, isotope tagging/MS/MS, or antibody arrays. Although it is outside the scope of this chapter to review comprehensively these techniques, we will go through a brief summary of some of them.

Simple adsorption/washing/desorption methods are fast, easily automated and have a widespread use (Table 5). In matrix-assisted laser desorption/ionization mass spectrometry (MALDI-TOF-MS) samples are digested with trypsin and deposited on the MALDI plate prior peptide mass fingerprinting (PMF) or partial sequencing of peptides (MS/MS; e.g., MALDI-TOF/TOF). In surface-enhanced laser desorption/ionization (SELDI) (e.g., ProteinChip ${ }^{\circledR}$, Ciphergen Biosystems, Palo Alto, CA) different chromatographic surfaces (hydrophobic, hydrophilic, etc) are used on a metal array to bind a subset of proteins from a sample. The unbound proteins are washed away, the bound proteins are overlaid with an organic matrix, and finally this fraction is profiled by MS analysis (e.g., MALDI-TOF-MS), generating rather simple mass spectra which can be easily analyzed. SELDI is a very useful method for high throughput screening, although this approach is not very reproducible and 
quite cumbersome to identify detected proteins, since require mass spectrometers such as FT-ICR-MS instruments to get a direct fragmentation of high molecular weight ions. However, patterns of peaks are usually found to be useful for the classification of pathological samples (Table 5).

Combination of online sample preparation with liquid chromatography (LC) and mass spectrometry (MS; LC-MS) is a common practice. Leaving aside the peptidomics approach, which is perhaps less interesting to study acute phase reactants, these medium-high molecular weight proteins can be analyzed through shotgun proteomics (Murakoshi et al., 2011). During the shotgun proteomics approach the entire serum/plasma proteome is submitted to trypsin digestion followed by peptides separation through one or normally (due to the very complex mixture of peptides) several liquid chromatography steps prior to MS. Multidimensional protein identification technology (MudPIT), for example, consists of a 2D chromatography separation prior to electrospray (ESI)-MS. In MudPIT the first dimension is normally a high loading capacity strong cation exchange (SCX) column, while the second dimension is reverse phase chromatography. After this extensive peptide fractionation and MS, the fragmentation data are matched against sequence databases using different software (e.g., SEQUEST) in order to identify serum/plasma proteins. However, several runs are required to get a comprehensive analysis of the whole proteome and, as with 2-DE, there are problems to mine the low abundant subproteome. In addition, qualitative over quantitative data are obtained by LC-MS, although the use of label-free LCMS profiling (Wesner et al., 2010) or techniques such as isotope-coded affinity tag (ICAT) (Lin et al., 2009), isobaric (iTRAQ) and MRM (multiple reaction monitoring; mTRAQ) tags for relative and absolute quantitation (Boylan et al., 2010; Kanq et al., 2010), or 18 O labelling (Qian et al., 2010) allows for quantitative comparisons between different samples. Finally, there are other high throughput methods to analyze the acute phase response in serum/plasma samples, such as capillary electrophoresis connected to electrospray ionization MS (CE-ESI-MS) or antibody arrays. Regarding antibody arrays, their main problem is that these methodologies are not discovery based; i.e., the only proteins that can be detected and quantitated are those targeted by the available antibodies. Moreover, several technological advances must be accomplished before truly high density antibody microarrays are readily available (Borrebaeck \& Wingren, 2009)

\subsection{Complexity and sensitivity: Strategies to enhance the detection of lower abundant APPs}

The unmasking of low abundant proteins (protein depletion), together with new technologies with enhanced resolving power, will provide access to more sensitive and specific biomarkers, and it is likely that some of them will represent new acute phase reactants. One way to reduce sample complexity and enhance the capacity to detect proteins or peptides at lower concentrations is to remove high-abundance proteins selectively. Only albumin (3.4-5.4 g/L) and immunoglobulins (IgG, 7.2-15 g/L; IgA, 0.9-0.33 g/L; IgM, 0.5$2.5 \mathrm{~g} / \mathrm{L}$; IgD, 0-0.4 g/L; IgE, 100-200 $\mu \mathrm{g} / \mathrm{L}$ ) account for $>70 \%$ of total serum proteins. Several affinity columns, based on dye ligands (e.g., Cibacron-blue) or specific antibodies (e.g., IgY, IgG), have been developed for albumin removal, while either thiophilic gels (Salgado, 2010) or resins with fixed antibodies (anti-IgG and, sometimes, even anti-IgA or anti-IgM) or immunoglobulin binding-proteins (protein $A$, protein $G$, protein $A / G$ mixtures, 
protein L) have been used for immunoglobulins substraction methods (Fang \& Zhang, 2008).

Although albumin is an acute phase reactant, removal of this abundant protein together with immunoglobulins facilitate the detection of less abundant or previously masked new acute phase reactants. A good example of this comes from our 2-DE analyses of human serum samples, where we were able to identify both leucine rich $\alpha 2$ glycoprotein (LRG) and apolipoprotein $\mathrm{H}(\mathrm{ApoH})$ (Salgado, 2010). LRG is a positive acute phase reactant whose concentration in serum (LRG; $\sim 3 \mu \mathrm{g} / \mathrm{ml}$ ) is close to the detection limit of 2-DE electrophoresis $(\mu \mathrm{g} / \mathrm{mL}$ range), and therefore very difficult to show in order to measure quantitative differences. The second one, apolipoprotein $\mathrm{H}$, has a position coincident with immunoglobulin $G$ heavy chains in 2-DE maps, appearing fully hidden prior albumin/immunoglobulins depletion (Salgado et al., 2010).

Sometimes, methods to eliminate albumin or immunoglobulins can be rather inespecific. For example, dye-ligand affinity columns (e.g, Cibracron Blue-based kits), still used in proteomic studies due to its relatively low cost, show a low specific binding for albumin and causes retention of unwanted proteins (Bellei et al., 2011). Thus, to achieve deeper and more specific protein depletion, different companies have developed several alternatives, like antibody immunoaffinity media or combinatorial peptide libraries. An example is the MARS-Hu-14 column (Agilent Technologies, Santa Clara, California, USA), intended to deplete the 14 most abundant proteins from serum or plasma samples. Another one is the ProteoPrep ${ }^{\circledR} 20$ Plasma Immunodepletion spin or LC-column from Sigma (St Louis, MO, USA), allowing the simultaneous depletion of 20 abundant proteins $(97-98 \%$ of plasma/serum proteins) and up to 50-fold increased protein loads (according to the company data). However, despite these clear benefits, the use of these affinity chromatography technologies generates great losses of information about the acute phase response, since all the removed proteins are acute phase reactants themselves. For example, the ProteoPrep ${ }^{\circledR} 20$ Plasma Immunodepletion column from Sigma eliminates albumin, IgG, transferrin, fibrinogen, IgA, a2- Macroglobulin, IgM, a1- Antitrypsin, complement C3, haptoglobin, apolipoprotein A1, A3 and B; a1-Acid Glycoprotein, ceruloplasmin, complement $\mathrm{C} 4, \mathrm{C} 1 \mathrm{q}$, IgD, transthyretin, and plasminogen.

Another choice to reduce sample complexity in order to study the acute phase response is to use ProteoMiner (Bio-Rad Laboratories) or ProSpectrum Libraries (Prolias). Both are approaches based upon a combinatorial peptide libraries displayed on beads. These beads are incubated with the starting material and are capable of interacting with most of proteins in serum/plasma proteome. Because the binding capacity of those beads is limited, and each peptide combination binds, in theory, to a unique protein sequence, the high-abundance proteins (e.g., albumin, immunoglobulins) quickly saturate the beads, so their excess can be washed away. Thus, low-abundance proteins are concentrated in the final sample, thereby decreasing the dynamic range of proteins and becoming a good alternative to immunoaffinity separation media. Using this technology, modification in APPs like apolipoprotein A1, apolipoprotein A4, antithrombin-III, C4a complement, vitamin D binding protein or apolipoprotein J can be easily traced (our unpublished data; Kolla et al., 2010).

Prefractionation is another choice to augment the depth of the analysis, even though at the expense of having to analyze multiple subsamples. One example is to select narrower $\mathrm{pH}$ 
ranges during the first dimension in 2-DE, to visualize only the part of the serum/plasma proteome we are interested in. Another way, perhaps not so popular, is to use preparative in-solution isoelectric focusing (e.g., Zoom IEF fractionator ${ }^{\circledR}$, Invitrogen) as the first step in body fluid analysis prior to 2-DE or, preferably, an automated method (e.g., LC-MS). Finally, the last strategy that we will mention to reduce sample complexity and cover more of the lower-abundance acute phase proteome is to enrich some protein families specifically. Thus, most of acute phase reactants are glycoproteins. Therefore, they can be enriched by a number of different techniques; one of the most commonly used is lectin affinity chromatography. More than 100 different lectins (concanavalin A, wheat germ agglutinin, peanut agglutinin, etc) are commercially available, which differ in specificity (a drawback of lectin chromatography). Glycoproteins are involved in many diseases (e.g., cancer), so the study of the O- and N-linked glycosilated APPs may therefore be particularly interesting (see below).

There is still another concern to address, which is associated with the removal of proteins like serum albumin or immunoglobulins. These high abundant proteins appear to fullfill the function of carriers for less abundant species, especially low molecular weight proteins (cytokines, growth factors, etc) that can only escape kidney clearance when bound to these high molecular weight carrier proteins. Moreover, some APPs, which are not so small, are also bound to these carrier proteins. Therefore, immunoaffinity subtraction may potentially remove non-targeted and potentially important APPs from serum/plasma samples, which may lead to unnoticed loses of information. In other words: what do we know about the interactome of APPs? For instance, it is known that transthyrretin binds another APP, the retinol binding protein (Folli et al., 2010), and the same happens with albumin (Gundry et al., 2007). In fact, almost every single APP has been described associated with albumin, like retinol-binding protein, $\alpha 2$-antiplasmin, complement factors, fibrinogen ( $\alpha$ chain), histidinerich glycoprotein, prothrombin, serum amyloid A4, $\alpha 1$-acid glycoproteins 1 and 2, $\alpha 1$ antichymotrypsin, $\alpha 1$-antitrypsin, $\alpha 1$ B-glycoprotein, $\alpha 2 \mathrm{HS}$-glycoprotein, antithrombin III, apolipoproteins, ceruloplasmin, haptoglobin, hemopexin, inter-a-trypsin inhibitor (heavy chain H4), leucine-rich $\alpha 2$ glycoprotein, paraoxonase 1, plasminogen, transferrin, transthyretin, vitamin D-binding protein or zinc- $\alpha 2$-glycoprotein (Gundry et al., 2007; Salgado et al., 2010). Immunoglobulins are another example, since they fix complement factors. Immunoglobulins-complement factors interactions may cause unwanted loss of the second ones during the process of removing antibodies from biological fluids, which could account for the reduced levels of complement factors $(\mathrm{C} 1, \mathrm{C} 3, \mathrm{C} 4)$ found in some clinic proteomic studies (Table 5). Finally, another good example comes from the association of haemoglobin and its scavenger protein haptoglobin with ApoA1 under an inflammatory context (Salvatore et al., 2007; Watanabe et al., 2009), so removal of haptoglobin can affect to ApoA1 detected levels, and vice versa. Despite these different setbacks, it can be stated that immunoaffinity depletion of just the two most abundant plasma proteins (i.e., albumin and immunoglobulins) still appears to be necessary, or at least beneficial, to enhance the resolving power (e.g., in 2-DE), reduce technical variation, detect or unmask lower abundant acute phase reactants (e.g, ceruloplasmin, C1q, ceruloplasmin, LRG) or reveal qualitative or quantitative differences in this group of plasma proteins more accurately. In any case, we always recommend analyze the retained fractions in order to extract more information from our clinical plasma/serum proteomic studies (Salgado et al., 2010). 
Another problem of studying the APP is their modulation across the time. As commented in the introduction, the so-called negative acute phase reactants are highly abundant proteins which are rapidly down-regulated in response to a variety of stimuli, (e.g., transthyretin). The same "stressors", in turn, cause the up-regulation of another important subclass: the positive APPs. This last class may be divided into three subclasses (I-III), based on their normal plasma concentrations. Class I, like ceruloplasmin $(0.3 \mathrm{mg} / \mathrm{mL})$ or complement factors C3 $(0.8-1.7 \mathrm{mg} / \mathrm{mL})$ or C4 $(0.15-0.65 \mathrm{mg} / \mathrm{mL})$, whose concentration may increase by $50 \%$; Class II ( $\alpha 1$-acid glycoprotein, $\sim 1 \mathrm{mg} / \mathrm{mL} ; \alpha 1$-antitrypsin, $2-5 \mathrm{mg} / \mathrm{mL} ; \alpha 1$ antichymotrypsin, $0.3-1.6 \mathrm{mg} / \mathrm{mL}$; haptoglobin, $0.3-0.2 \mathrm{mg} / \mathrm{mL}$; fibrinogen, $2-6 \mathrm{mg} / \mathrm{mL}$ ) begin to increase 24 to 48 hours and reach to their maximum level (two to five fold enhancement) in about 7-10 days, and require about two weeks to return to their normal levels; and finally class III (e.g, C reactive protein/CRP $<5 \mu \mathrm{g} / \mathrm{mL}$; serum amyloid A/SAA; $<10 \mu \mathrm{g} / \mathrm{mL}$ ), whose concentrations rise as early as 4 hours after inflammatory stimulus and attain their maximum levels within 24 to 72 hours (concentration may increase up to 1000 fold) and also decline very rapidly. Thus, depending on the acute/chronic nature of the pathology or the sampling time, one may get different results.

\subsection{Clinical proteomics}

The field of clinical proteomics holds tremendous potential for discovery of noninvasive diagnostic and prognostic biomarkers or the identification of novel drugs targets, although there is a lack of approved IVD biomarkers (Table 4) based on clinical proteomics data (Anderson, 2010). However, scientists have accumulated during the last years a huge amount of high-throughput proteomic data with a true translational potential. Table 5 summarizes a literature review on different proteomics strategies used for the study of serum/plasma samples from several important diseases, as well as an overview on the major APPs alterations observed.

There are at least three sources of plasma/serum biomarkers: a) the primary diseased tissue, such as endothelial cells in atherosclerosis or neoplastic cells in cancer; b) the microenvironment around the primary cells; c) the systemic response to these local alterations, where APPs are involved. APPs are altered in bacterial infections, fractures, tumors, Crohn's disease, surgery, rheumatoid arthritis,burns or systemic vasculitis; however, they remain unaltered in others. The use of this third source of biomarkers has a clear advantage, as the APR is a kind of "amplificated biological response" (Omenn et al., 2007). Maybe for that reason, most of candidate biomarkers discovered in clinical proteomic studies using biofluids (plasma, serum, urine, cerebrospinal fluid, pleural effusion, etc) are APPs, which are shared among different pathological situations, like different cancers or autoimmune diseases. Therefore, contrary to single proteins biomarkers like PSA (prostate cancer), CA 125 (ovarian cancer), CEA (pancreatic cancer or CA 19-9 (breast cancer), which show a low sensitivity and specificity during disease detection, acute phase reactants (for example, C-reactive protein and cardiovascular risk detection) are more sensitive, but lack specificity. For example, Table 5 shows that CRP is elevated in allogeneic hematopoietic stem cell transplantation/HSCT, Down syndrome (maternal plasma), or hepatocellular carcinoma. Similarly, alpha 1 acid glycoprotein, alpha 1-antichymotrypsin, $\alpha 1$ - B-Glycoprotein, antithrombin III, apoA1, ceruloplasmin, leucine rich glycoprotein, prothrombin, serum amyloid A, serum amyloid P component, 
transferrin, transthyretin, vitamin D Binding Protein or zinc $\alpha 2$-glycoprotein are systematically either down- or up-regulated in different diseases (low specificity) (Table 5). This fact does not mean they are not useful as biomarkers. Rheumatoid arthritis (RA) is an autoimmune disease affecting $0.5-1 \%$ of adults characterized by persistent synovitis, systemic chronic inflammation and presence of autoantibodies. About $50-80 \%$ of RA patients are seropositive for rheumatoid factor (RF; IgM and IgA autoantibodies directed against the Fc region of IgG), antibodies against cyclic citrullinated peptides (anti-CCP), or both. Anti-CCP antibodies are more sensitive and specific for RA diagnosis than RF, which is present in up to $20 \%$ of elderly individuals, and also seem to be a better predictor of progressive joint destruction (Scott et al., 2010). Early diagnosis of RA is fundamental to achieve significantly better clinical outcomes, and acute-phase reactants can help to assess tha probability of developing RA once there are evidences of inflammatory arthritis. Indeed, the low specific C-reactive protein is part of the ACR (American College of Rheumatology)/EULAR (European League Against Rheumatism) 2010 criteria to classify both early and established disease. Also on this regard, in 2010 our group published a preliminary 2-DE study using Immunoglobulins/HSA-depleted serum samples from healthy individuals and recently diagnosed/untreated rheumatoid arthritis (RA) patients. This work found that leucine rich glycoprotein was significantly upregulated, while antithombin III was downmodulated (Salgado et al., 2010). Separately, Satoshi Serada (Serada et al., 2010), using iTRAQ, found that leucine rich glycoprotein is increased in RA patients before therapy. Like C-reactive protein, leucine rich glycoprotein is a low specific biomarker, as elevations of this APP are observed in bacterial (toxic shock syndrome) and viral (HIV) infections, autoimmune diseases (Crohn's disease, Behcet's disease) and some kind of cancers. However, this novel biomarker may be particularly useful to monitor disease activity in patients with active disease but normal C-reactive protein levels (Serada et al., 2010). In clear contrast (Table 5), other APPs, such as alpha 1 antitrypsin, hemopexin and, curiously, haptoglobin (considered a positive acute phase reactant), show a higher variability. Perhaps, such variability is rather due to genetic or posttranslational based changes more than simple augmented or reduced levels (see below), but there is not doubt that this kind of APPs may be more specific and therefore attractive from a diagnostic point of view.

Anyhow, the simple test paradigm, where one expects changes in the concentration of unique biomarkers linked to the diagnosis of single diseases, is gradually moving on to the use of multivariate tests. In classical clinical chemistry, a biomarker determination has diagnostic value only if compared with a reference interval including 95\% (about 2 standard deviations above and below the mean value) of the reference values from the reference population (normally "healthy" people, but not always). Thus, plasma levels of a particular disease biomarker should lie within that reference interval, and values outside that reference range could point out (in theory) a pathological situation. However, this is not so straightforward. As mentioned above, it seems not likely to find a fully specific acute phase protein, since other diseases may alter the blood levels of this biomarker too (Table 5). What is more, acute phase protein concentrations within nondiseased subjects may vary substantially and overlap with those within diseased populations because: (A) genetic influences, like gender or race; (B) non genetic influences, like age, exercise, circadiam rhythm, season, smoking, diet or sleep; (C) 
medical treatment, such as drug therapy; and (D) pre-analytical phase variation, including sample drawing (body posture, time of venous occlusion by the tourniquet), transport and preparation. Indeed, there is a strong evidence of genetic control of plasma protein abundance: $\sim 12-95 \%$ of the quantitative variation in specific plasma protein levels is genetic in origin (Anderson \& Anderson, 2002). Therefore, it is important to control both the analytical and biological variation in any clinical proteomic study of the acute phase response in order to predict the number of samples and replicates that should be analyzed to find statistically significant differences (Hunt et al., 2005).

As we have just mentioned, most biomarker studies in scientific literature detecting acute phase response proteins are based on transversal/cross-sectional comparisons (e.g., healthy vs diseased group; Table 5). This approach suffers sometimes from large biological variation in the baseline values of APPs within the reference population, which, summed to the technical variation, generate a significant overlap with the concentration range of diseased populations. This overlap may mask the existence of significant changes and leads to low sensitivity (false negatives) and, especially for APPs, low specificity (false positives), once a cut-off value is set. ROC curves can help us to select such a discriminative value in order to maximize the specificity without renouncing to a good sensitivity level. Nonetheless, even if we find a sensitive and specific acute phase protein for a disease, the positive predictive value of that biomarker would depend, in the end, on the disease's prevalence, since even specificities as high as $99 \%$ will result in false positive results of low prevalent pathologies (Hoffman et al., 2007).

To solve these problems, two changes may help. The first one is the use of individual instead of population-based reference intervals for APPs (personalized medicine). Different authors have monitored the protein expression dynamics that take place within one individual to overcome all these limitations and to get more meaningful data; for example, to reveal serum/plasma biomarkers or to predict the recovery or treatment response during acute events such as trauma, infection or drug intervention. An example is the study of Han Roelofsen in 2007, who studied the kinetic of serum proteome before and after colon laparoscopic surgery (Roelofsen et al., 2007). Using SELDI-TOF-MS, this group could distinguish up to four groups of proteins based on their expression pattern kinetics. Thus, they identified serum amyloid protein and C-reactive protein as part of the positive slow response cluster, and transthyrretin as a negative reactant belonging to the fast response group. These authors were also able to observe serum amyloid protein differences between patients, related to their recovery from surgery (Roelofsen et al., 2007). Another interesting work was the recent study on the serum proteome changes produced during allo hematopoietic stem cell transplantation by Joohyun Ryu (Ryu et al., 2010). The aim of this work was to find biomarkers suited for the diagnosis and follow-up of patients who experience complications after allo-HSCT (Table 5). Using 2DE analysis of HSA/IgG depleted sera obtained at different times (pretransplant, 7, 14 and 21 days), these authors found 14 differentially expressed APPs. They observed three different expression patterns: A) serum amyloid P, ApoE and C-reactive protein, elevated 14 or 21 days post-HSCT; B) haptoglobin, alpha 2 HS glycoprotein, decreased upon HSCT; C) and APPs with irregular patterns. Based on their data, this group has suggested CRP as a risk factor for the development of major transplant-related complications, and haptoglobin as a prognostic biomarker of relapses in underlying 
hematologic disease (Ryu et al., 2010). On the other hand, the second change consist in using multiplex panels of specific APPs (a proteome signature), which may improve diagnostic performance through the use of protein ratios (Gruys et al., 2006) or more sophisticated interpretive algorithms. In time, some of acute phase response biomarkers combinations might perhaps mature to FDA/EMEA-approvable IVD-tests, something costly and time-consuming ( $\sim 10-20$ years).

As commented above, $12-95 \%$ of the quantitative variation in specific plasma protein levels depends on genetic background. Thus, haptoglobin shows a $20 \% \mathrm{CV}$ intraindividual, and 27.9\% CV interindividual, and C-reactive protein $42.2 \% \mathrm{CV}$ intraindividual and $92.5 \% \mathrm{CV}$ interindividual (Anderson \& Anderson, 2002). Polymorphic APPs, such as haptoglobin, alpha 2 HS glycoprotein, alpha 1 antitrypsin, vitamin D binding protein, and transferrin, have been detected by proteomic techniques (2-DE) since the seventies (Anderson \& Anderson, 1977; Goldman et al., 1985; Salgado et al, 2010). Some of these variations have been associated with several pathological conditions. For example, schizophrenia shows enhanced levels of haptoglobin (Table 5) and a significant association with the HP 1-2 genotype (Wan et al., 2007), while the Hp 2-2 genotype seems rather linked to increased hemoglobin/haptoglobin/hemopexin content on HDL (ApoA1) particles and higher risk of coronary heart disease (Watanabe et al., 2009). It also has been found a higher susceptibility to chronic graft versus host disease (GVDH) after allogeneic hematopoietic cell transplantation (HSCT) in HP 2-2 patients (McGuirk et al., 2009). On the other hand, it was detected a higher incidence of myotrophic lateral sclerosis in vitamin D binding protein GC2 isoform carriers (Palma et al., 2008).

Post-translational modifications (PTMs) have also an important role in determining the function of proteins and come in a great degree of variation: citrullination, phosphorylation, proteolysis, glycosylation, oxidative modifications, etc. Many APPs show a complex combination of post-translational modifications, which may be diseaseor inflammation-dependent. Thus, analysis of serum glycome in chronic inflammation associated to advanced ovarian cancer patients (Saldova et al., 2007) revealed sialyl Lewis $x\left(\mathrm{SLe}^{\mathrm{x}}\right)$ structures in APPs that already had this marker: haptoglobin $\beta$-chain, alpha 1 acid glycoprotein, and alpha 1 antichymotrypsin. These glycosylation changes were parallel to the upregulation of these proteins (Amon et al., 2010; Lin et al, 2009) (Table 5), and could be tumour specific (Saldova et al., 2007). In addition, PTMs may have an impact on 2-DE patterns (Butler et al., 2003; Saldova et al., 2007), and application of lectin affinity chromatography prior 2-DE (or other proteomic techniques) could sometimes unmask previously unnoted expression changes (Seriramalu et al., 2010). Some of those pathological-dependent post-translational modifications in APPs may result in the generation of new antigens (neo-antigenicity), and hence autoantibodies against them (autoimmune diseases). Two good examples to illustrate this point are autoantibodies specific for citrullinated proteins in rheumatoid arthritis/multiple sclerosis and autoantibodies recognizing oxidatively modified C1q in rheumatoid arthritis and systemic erythematosus lupus (Eggleton, 2008). Similarly, phospholipids and apolipoproteins (e.g., ApoB100) of low-density lipoprotein (LDL) are also susceptible to oxidation, promoting chronic inflammation and the generation of autoantibodies against highly immunogenic oxidation-specific neopitopes (Eggleton, 2008). 


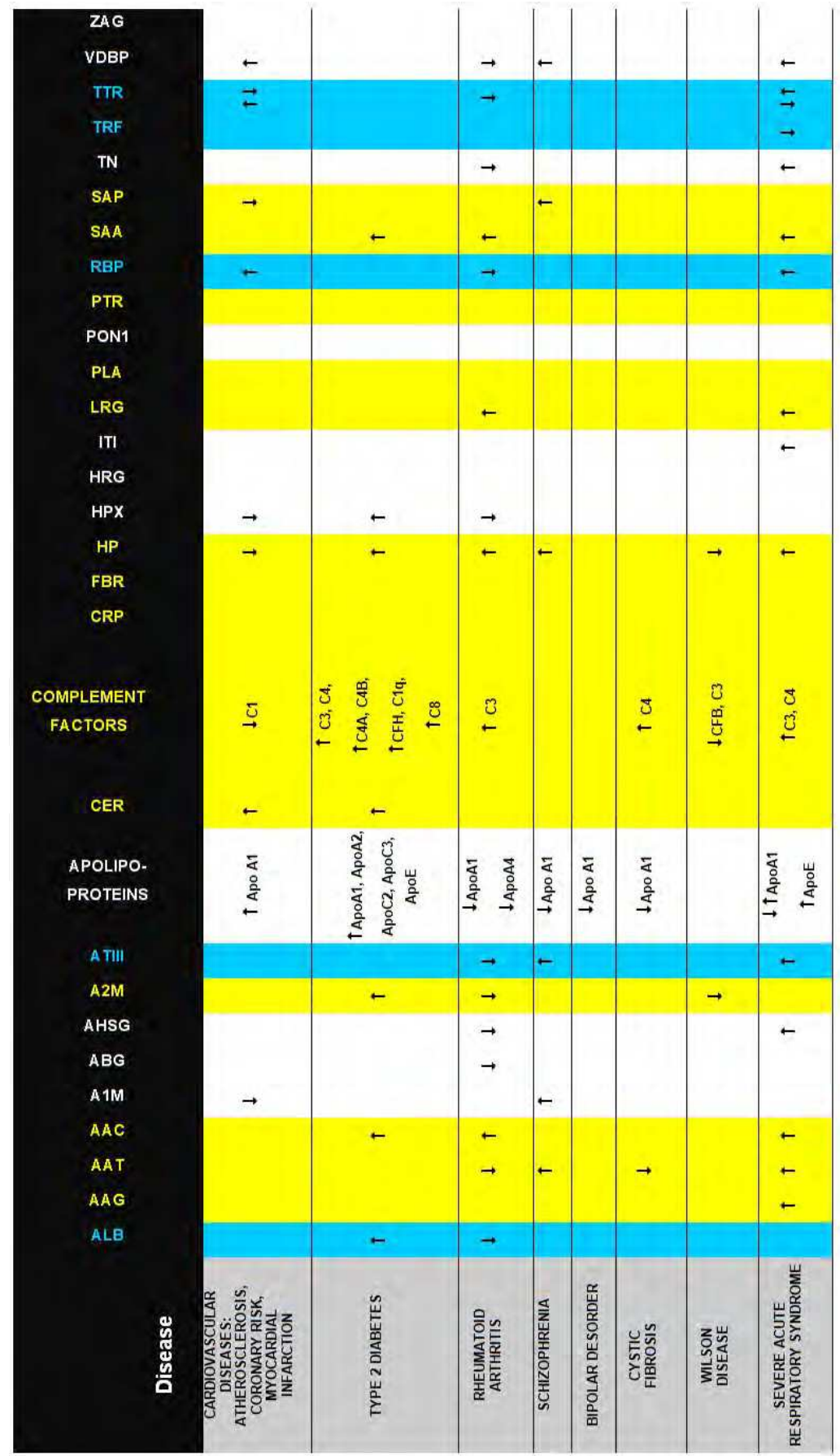




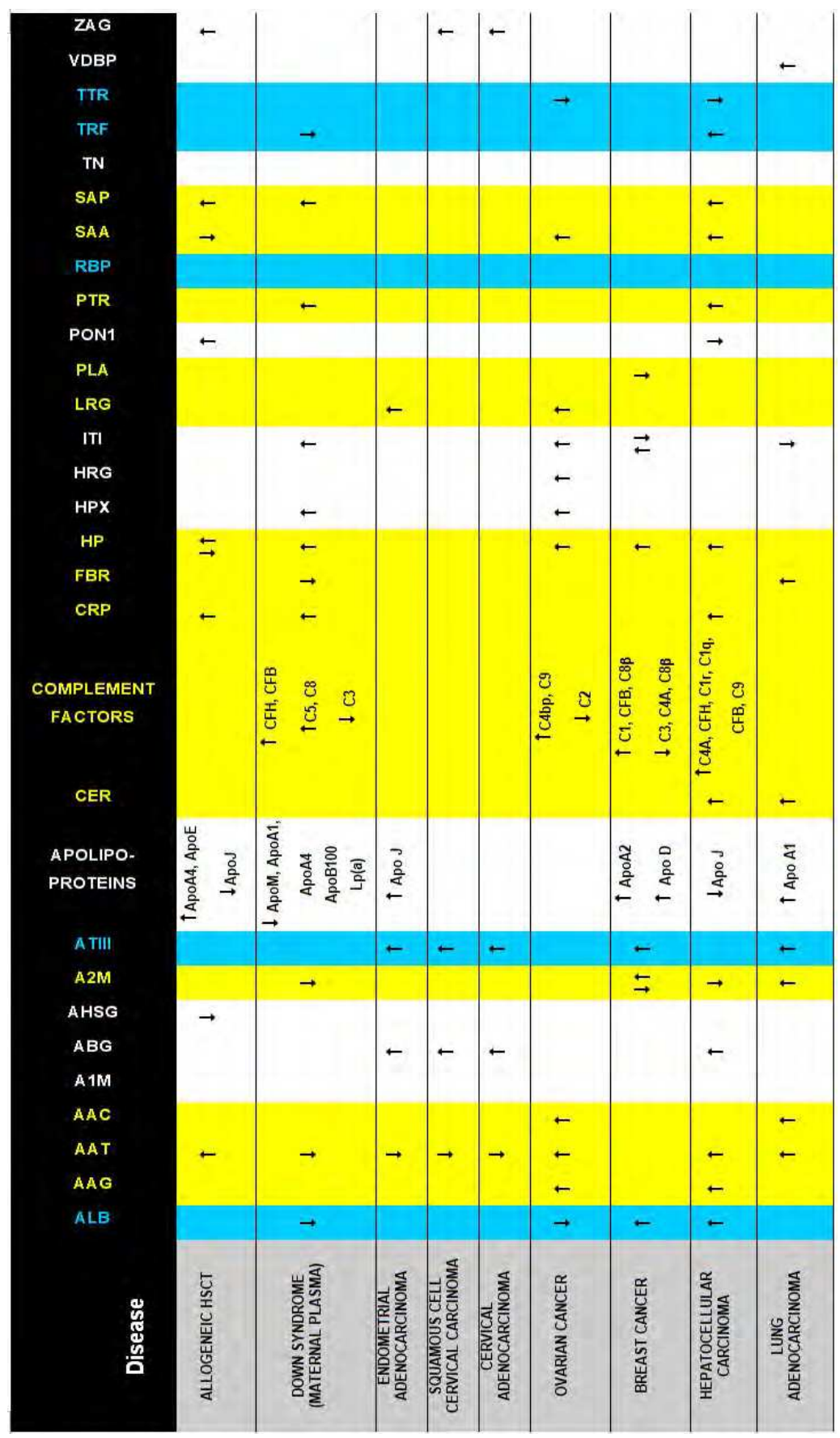

Table 5 . Acute phase protein patterns revealed by proteomic studies in different diseases. 
Sometimes, different studies on a particular disease yield opposite data regarding the same acute phase reactant, and there are examples even within the same paper: e.g., alpha 2 macroglobulin, inter- $\alpha$-trypsin inhibitor in breast cancer, and transthyretin in severe acute respiratory syndrome. In the last case, this is due to different modulation of low a high molecular weight forms. Abbreviations are the same as in Table 1. Blue colour indicates prototypical positive acute phase proteins, while yellow colour points out characteristic negative acute phase reactants. Proteomic methodologies used: Cardiovascular disease (Kim et al., 2011) 2-DE. Silver staining. MS/MS. Western blot. ELISA. Type 2 diabetes (Li et al., 2008) 1-DE/Coomassie. Rheumatoid arthritis (Salgado et al., 2010) HSA/Ig depleted sera. 2-DE/Coomassie. MS/MS ; (Doherty et al., 1998) whole plasma. 2-DE/Coomassie. Immunoassays; (Serada et al., 2010) Sera were depleted with MARS-Hu-14 (14 abundant proteins removed, Agilent Technologies), iTRAQ labelling. nanoLC-MS/MS. ELISA. Schizophrenia (Wan et al. 2007) 2-DE/Coomassie. MALDI-MS (PMF). Bipolar disorder (Sussulini et al., 2011) SELDI-TOF. Cystic fibrosis (Charro et al., 2011) Pooled serum samples processed with Multiple Affinity Removal System (MARS) spin cartridges (6 abundant proteins removed; Agilent Technologies). 2-DE/Coomassie. MALDI-TOF/TOF (MS/MS). Shotgun proteomics (nanoRPLC-MS/MS). Wilson disease (Park et al., 2009) Sera were depleted using MARS column (6 abundant proteins removed, Agilent Technologies). 2-DE/Coomassie. MALDI-TOF (MS), MS/MS and Western blot. Severe acute respiratory syndrome (Chen et al., 2004) Unfractionated plasma samples. 2-DE/Sypro Ruby. MALDITOF-MS and LC-MS/MS; (Wan et al., 2006) HSA/IgG depleted pooled plasma samples. 2DE DIGE. MALDI-MS/MS and Western blot. Allogeneic HSCT (Ryu et al., 2010) HSA/IgG depleted sera. 2-DE/silver staining. L-MS/MS. (McGuirk et al., 2010) HSA depleted sera. 2DE/Silver staining. LC-MS/MS. Down syndrome (Kolla et al., 2010) Plasma samples depleted with ProteoMiner Enrichment Kit (BioRad). Trypsin digestion, iTRAQ labelling, and SCX/Nano LC MALDI-TOF-TOF (MS/MS). Endometrial adenocarcinome (AbdulRahman et al., 2007). Unfractionated sera, 2-DE/ Silver Stain/Coomassie. MALDI-MS (PMF and MS/MS). Western blot. Competitive ELISA. Squamous cell cervical carcinoma (AbdulRahman et al., 2007). Unfractionated sera, 2-DE/ Silver Stain/Coomassie. MALDI-MS (PMF and MS/MS). Western blot. Competitive ELISA. Cervical adenocarcinoma (Abdul-Rahman et al., 2007). Unfractionated sera, 2-DE/ Silver Stain/Coomassie. MALDI-MS (PMF and MS/MS). Western blot. Competitive ELISA. Ovarian cancer (Amon et al., 2010) Serum samples were depleted using Multiple Affinity Removal System (MARS-7) columns (Agilent). 2D (Anion exchange/Reverse phase)-LC. MS/MS and ELISA. (Lin et al., 2009) Depleted sera (IgY12 columns). ICAT/ $\mu \mathrm{LC} / \mathrm{MS} / \mathrm{MS}$ and ELISA. (Boylan et al., 2010) Depleted sera (MARS and IgY12 columns). Trypsin digestion, iTRAQ labelling, and SCX/reverse phase LC/MS/MS. Western blot. Breast cancer (Kadowaki et al., 2011) MARS6 (Agilent) depleted sera. Reverse phase HPLC followed by 2-DE/Coomassie. Western blot and ELISA. Hepatocellular carcinoma (Yang et al., 2007) Serum samples. 2-DE/silver staining. Nano-HPLC-ESI-MS/MS. Lung adenocarcinoma (Hongsachart et al., 2009) Crude sera or WGA lectin-bound serum proteins. 2-DE/Sypro and 2-DE-DIGE. MALDI-TOF MS and MS/MS. Western blot.

\section{Conclusion}

The acute phase response is a highly conserved system that takes place during inflammation. During this response the serum levels of a continuously growing list of 
plasma proteins change, either up- (some of them even 1000 fold) or downwards, under the influence of cytokines like IL-1, IL-6 or TNF $\alpha$. Some APPs have antiinflammatory effects (e.g., C-reactive protein, leptin), while others influence leukocyte activation/trafficking (e.g., serum amyloid A), modulate the coagulation/complement cascade (e.g., anti-thrombin 3, Creactive protein) or work for example as scavenger proteins (e.g., haptoglobin, serum amyloid A). With such a variety of functions, it is not surprising to find that some of these APPs are useful for diagnosis and prognosis of different diseases, and for that reason different companies have developed a high number of IVD tests based on single acute phase reactants. For example, amongst others, APPs-based tests are being used nowadays in hepatic/kidney function or nutritional status evaluation, to check the presence of infectious diseases, inflammation or autoimmune diseases, or to detect anemia or thrombofilia/bledding disorders. Apart from the discovery of new APPs whose potential clinical interest awaits future studies, some older ones seem to have got a second life as biomarkers, like C-reactive protein and cardiovascular risk assesment. In addition, the role of many of these APPs has not been completely elucidated (e.g., leucine rich $\alpha 2$ glycoprotein, serum amyloid A), and experimental evidences also point out new associations of some of these proteins with different diseases, like for instance serum amyloid A and obesity-related disorders (e.g., cardiovascular diseases, atherosclerosis, diabetes, insulin resistence) (Zhao et al., 2010). Therefore, it can be said that much work remains to be done in the future around the acute phase reactants field. Actually, body fluid proteomics techniques have become promising tools to uncover new APPs and their associations with human disorders, and it is likely that some of these proteomic studies will finally reveal proteins within the expanding group of APPs with potential clinical interest. Moreover, these findings might even lead to the development and marketing of new IVD tests by the biotechnology industry. Nevertheless, according to data from different pathological scenarios, it is more likely that combinations of APPs will characterize disease states or predict disease outcomes better than single APPs. Thus, what the future may hold is the use of IVD assays with improved tests properties (e.g., sensitivity, specificity) based on disease-associated acute phase reactants patterns (or APPs "signatures").

\section{References}

Abdul-Rahman, P.S.; Lim, B.K. \& Hashim, O.H. (2007). Expression of High-abundance Proteins in Sera of Patients with Endometrial and Cervical Cancers: Analysis Using 2-DE with Silver Staining and Lectin Detection Methods. Electrophoresis, Vol.28, No.12, (June 2007), pp. 1989-1996, ISSN 1522-2683

Adkins, J.N.; Varnum, S.M.; Auberry, K.J.; Moore, R.J.; Angell, N.H.; Smith, R.D.; Springer, D.L. \& Pounds, J.G. (2002). Toward a Human Blood Serum Proteome: Analysis by Multidimensional Separation Coupled with Mass Spectrometry. Molecular and Cell Proteomics, Vol.1, No.12, (December 2002), pp. 947-955, ISSN 1535-9484

Aked, D.M., \& Foster, S.J. (1987). Leukotriene B4 and Prostaglandin E2 Mediate the Inflammatory Response of Rabbit Skin to Intradermal Arachidonic Acid. British Journal of Pharmacology, Vol.92, No.3, (November 1987), pp. 545-552, ISSN 0007-118

Amon, L.M.; Law, W.; Fitzgibbon, M.P.; Gross, J.A.; O'Briant, K.; Peterson, A.; Drescher, C.; Martin, D.B. \& McIntosh, M. (2010). Integrative Proteomic Analysis of Serum and Peritoneal Fluids Helps Identify Proteins that are Up-regulated in Serum of Women with Ovarian Cancer. PLoS One, Vol.5, No.6, (June 2010), e11137, ISSN 1932-6203 
Anderson, L. \& Anderson, N.G. (1977). High resolution Two-dimensional Electrophoresis of Human Plasma Proteins. Proceedings of the National Academy of Sciences of the United States of America, Vol.74, No.12, (December 1977), pp. 5421-5425, ISSN 0027-8424

Anderson, N.L. \& Anderson, N.G. (2002). The Human Plasma Proteome. Molecular and Cell Proteomics, Vol.1, No.11, (November 2002), pp. 845-867, ISSN 1535-9484

Anderson, N.L.; Polanski, M.; Pieper, R.; Gatlin, T.; Tirumalai, R.S.; Conrads, T.P.; Veenstra, T.D.; Adkins, J.N.; Pounds, J.G.; Fagan, R. \& Lobley, A (2004). The Human Plasma Proteome: a Nonredundant List Developed by Combination of Four Separate Sources. Molecular and Cell Proteomics, Vol.3, No.4, (April 2004), pp. 311-326, ISSN 1535-9484

Anderson, N.L. (2010). The Clinical Plasma Proteome: a Survey of Clinical Assays for Proteins in Plasma and Serum. Clinical Chemistry, Vol.56, No.2, (February 2010), pp. 177-185, ISSN 0009-9147

Baud, V. \& Karin, M. (2001). Signal Transduction by Tumor Necrosis Factor and its Relatives. Trends in Cell Biology Vol.11, No.9, (September 2001), pp. 372-377, ISSN 0962-8924

Baumann, H \& Gauldie, J. (1994). The Acute Phase Response. Immunology Today, Vol.15, No. 2, (February 1994), pp. 74-80, ISSN 0167-5699

Bellei, E.; Bergamini, S.; Monari, E.; Fantoni, L.I.; Cuoghi, A.; Ozben, T. \& Tomasi, A. (2011). High-abundance Proteins Depletion for Serum Proteomic Analysis: Concomitant Removal of Non-targeted Proteins. Amino Acids, Vol.40, No.1, (January 2011), pp. 145-156, ISSN 1438-2199

Benigni, F.; Fantuzzi, G.; Sacco, S.; Sironi, M.; Pozzi, P.; Dinarello, C.A.; Sipe, J.D.; Poli, V.; Cappelletti, M.; Paonessa, G.; Pennica, D.; Panayotatos, N. \& Ghezzi, P. (1996). Six Different Cytokines that Share GP130 as a Receptor Subunit, Induce Serum Amyloid A and Potentiate the Induction of Interleukin- 6 and the Activation of the Hypothalamus-pituitary-adrenal Axis by Interleukin-1. Blood, Vol.87, No.5, (March 1996), pp. 1851-1854, ISSN 1528-0020

Besedovsky, H.; del Rey, A.; Sorkin, E. \& Dinarello, C. (1986). Immunoregulatory Feedback between Interleukin-1 and Glucocorticoid Hormones. Science, Vol.233, No.4764, (August 1986), pp. 652-654, ISSN 1095-9203

Bevilacqua, M.P.; Pober, J.S.; Majeau, G.R.; Cotran, R.S. \& Gimbrone, M.A. (1984). Interleukin 1 (IL-1) Induces Biosynthesis and Cell Surface Expression of Procoagulant Activity in Human Vascular Endothelial Cells. The Journal of Experimental Medicine, Vol.160, No.2, (August 1984), pp. 618 -623, ISSN 1540-9538

Borrebaeck, C.A. \& Wingren, C. (2009). Design of High-density Antibody Microarrays for Disease Proteomics: Key Technological Issues. Journal of Proteomics, Vol.72, No.6, (August 2009), pp. 928-935, ISSN 1874-3919

Boylan, K.L.; Andersen, J.D.; Anderson, L.B.; Higgins, L. \& Skubitz, A.P. (2010). Quantitative Proteomic Analysis by iTRAQ(R) for the Identification of Candidate Biomarkers in Ovarian Cancer Serum. Proteome Science, Vol.8, (June 2010), pp. 31, ISSN 1477-5956

Brom, J. \& König, W. (1992). Cytokine-induced (Interleukins-3, -6 and -8 and Tumour Necrosis Factor-beta) Activation and Deactivation of Human Neutrophils. Immunology, Vol.75, No.2, (February 1992), pp. 281-285, ISSN 1365-2567 
Butcher, E.C. (1991). Leukocyte-endothelial Cell Recognition: Three (or More) Steps to Specificity and Diversity. Cell, Vol.67, No.6, (December 1991), pp. 1033-1036, ISSN 0092-8674

Butler, M.; Quelhas, D.; Critchley, A.J.; Carchon, H.; Hebestreit, H.F.; Hibbert, R.G.; Vilarinho, L.; Teles, E.; Matthijs, G.; Schollen, E.; Argibay, P.; Harvey, D.J.; Dwek, R.A.; Jaeken, J.\& Rudd, P.M. (2003). Detailed Glycan Analysis of Serum Glycoproteins of Patients with Congenital Disorders of Glycosylation Indicates the Specific Defective Glycan Processing Step and Provides an Insight into Pathogenesis. Glycobiology, Vol.13, No. 9, (September 2003), pp. 601-622, ISSN 09596658

Charro, N.; Hood, B.L.; Faria, D.; Pacheco, P.; Azevedo, P.; Lopes, C.; de Almeida, A.B.; Couto, F.M.; Conrads, T.P. \& Penque, D. (2010). Serum Proteomics Signature of Cystic Fibrosis Patients: a Complementary 2-DE and LC-MS/MS Approach. Journal of Proteomics, Vol. 74, No.1, (January 2011), pp. 110-126, ISSN 1874-3919

Chen, J.H.; Chang, Y.W.; Yao, C.W.; Chiueh, T.S.; Huang, S.C.; Chien, K.Y.; Chen, A.; Chang, F.Y.; Wong, C.H. \& Chen, Y.J. (2004). Plasma Proteome of Severe Acute Respiratory Syndrome Analyzed by Two-dimensional Gel Electrophoresis and Mass Spectrometry. Proceedings of the National Academy of Sciences of the United States of America, Vol.7, No.49, (December 2004), pp. 17039-17044, ISSN 0027-8424

Cines, D.B.; Pollak, E.S.; Buck C.A.; Loscalzo, J.; Zimmerman, G.A.; McEver, R.P.; Pober, J.S.; Wick, T.M.; Konkle, B.A.; Schwartz, B.S.; Barnathan, E.S.; McCrae, K.R.; Hug, B.A.; Schmidt, A.M. \& Stern, D.M. (1998). Endothelial Cells in Physiology and in the Pathophysiology of Vascular Disorders. Blood, Vol.91, No.10, (May 1998), pp. 35273561, ISSN 1528-0020

Colley, C.M.; Fleck, A.; Goode, A.W.; Muller, B.R. \& Myers, M.A. (1983). Early Time Course of the Acute Phase Protein Response in Man. Journal of Clinical Pathology, Vol.36, No. 2, (February 1983), pp. 203-207, ISSN 1472-4146

Collins, R.A. \& Grounds, M.D. (2001). The Role of Tumor Necrosis Factor-alpha (TNF-a) in Skeletal Muscle Regeneration. Studies in TNF-alpha(-/-) and TNF-alpha(-/-)/LTalpha(-/-) mice. Journal of Histochemistry \& Cytochemistry, Vol.49, No.8, (August 201), pp. 989-1001, ISSN 1551-5044

Coussens, L.M. \& Werb, Z. (2002). Inflammation and Cancer. Nature, Vol.420, No.6917, (December 2002), pp. 860-867, ISSN 1476-4687

Dantzer, R. (2001). Cytokine-induced Sickness Behavior: Where do We Stand? Brain, Behavior, and Immunity, Vol.15, No.1 (March 2001), pp. 7-24, ISSN 0889-1591.

Deboer, T.; Fontana A. \& Tobler I. (2002). Tumor Necrosis Factor (TNF) Ligand and TNF Receptor Deficiency Affects Sleep and the Sleep EEG. Journal of Neurophysiology, Vol.88, No.2, (August 2002), pp. 839-846, ISSN 1522-1598

Dinarello, C. (1988). Biology of Interleukin 1. The FASEB Journal, Vol.2, No.2 (February 1988), pp108-115, ISSN 1530-6860

Dinarello, C.A. \& Wolff S.M. (1993). The Role of Interleukin-1 in Disease. The New England Journal of Medicine, Vol.328, No.2, (January 1993), pp. 106-113, ISSN 1533-4406

Doherty, N.S.; Littman, B.H.; Reilly, K.; Swindell, A.C.; Buss, J.M. \& Anderson, N.L. (1998). Analysis of Changes in Acute-phase Plasma Proteins in an Acute Inflammatory Response and in Rheumatoid Arthritis using Two-dimensional Gel Electrophoresis. Electrophoresis, Vol.19, No.2, (February 1998), pp. 355-363, ISSN 1522-2683 
Dowton, S.B. \& Colten, H.R. (1988). Acute Phase Reactants in Inflammation and Infection. Seminars in Hematology, Vol.25, No.2, (April 1988), pp. 84-90, ISSN 1532-8686

Duncan, M.R. \& Berman, B. (1989). Differential Regulation of Collagen, Glycosaminoglycan, Fibronectin, and Collagenase Activity Production in Cultured Human Adult Dermal Fibroblasts by Interleukin 1-Alpha and Beta and Tumor Necrosis FactorAlpha and Beta. Journal of Investigative Dermatol, Vol.92, No.5 (May 1989), pp. 699706, ISSN 1523-1747

Eggleton, P.; Haigh, R. \& Winyard, P.G. (2008). Consequence of Neo-antigenicity of the "Altered Self". Rheumatology, Vol.47, No.5, (May 2008), pp. 567-571, ISSN 1462-0324

Fang, J.; Wang, Y. \& Krueger, J.M. (1997). Mice Lacking the TNF 55 kDa Receptor Fail to Sleep More After TNFalpha Treatment. The Journal of Neuroscience: The Official Journal of the Society for Neuroscience, Vol.17, No.15, (August 1997), pp. 5949-5955.

Fang, X. \& Zhang, W.W. (2008). Affinity Separation and Enrichment Methods in Proteomic Analysis. Journal of Proteomics, Vol.71, No.3, (August 2008), pp. 284-303, ISSN 18743919

Feingold, K.; Doerrler, W.; Dinarello, C.; Fiers, W., \& Grunfeld, C. (1992). Stimulation of Lipolysis in Cultured Fat Cells by Tumor Necrosis Factor, Interleukin-1, and the Interferons is Blocked by Inhibition of Prostaglandin Synthesis. Endocrinology, Vol.130, No.1, (January 1992), pp. 10-16, ISSN 1945-7170

Fiocchi, C. (1998). Inflammatory Bowel Disease: Etiology and Pathogenesis. Gastroenterology, Vol.115, No.1, (July 1998), pp. 182-205, ISSN 0016-5085

Folli, C.; Favilla, R. \& Berni, R. (2010). The Interaction between Retinol-binding Protein and Transthyretin Analyzed by Fluorescence Anisotropy. Methods in Molecular Biology, Vol.652, pp. 189-207, ISSN 1064-3745

Goldman, D.; Goldin, L.R.; Rathnagiri, P.; O'Brien, S.J.; Egeland, J.A. \& Merril, C.R. (1985). Twenty-seven Protein Polymorphisms by Two-dimensional Electrophoresis of Serum, Erythrocytes, and Fibroblasts in Two Pedigrees. The American Journal of Human Genetics, Vol.37, No.5, (September 1985), pp. 898-911, ISSN 002-9297

Gross, T.J.; Leavell, K.J. \& Peterson, M.W. (1997). CD11b/CD18 Mediates the Neutrophil Chemotactic Activity of Fibrin Degradation Product D Domain. Thrombosis and Haemostasis, Vol.77, No.5 (May 1997), pp. 894-900, ISSN 0340-6245

Gruys, E.; Toussaint, M.J.; Niewold, T.A.; Koopmans, S.J.; van Dijk, E.\& Meloen, R.H. (2006). Monitoring Health by Values of Acute Phase Proteins. Acta histochemica, Vol.108, No.3, (May 2006), pp. 229-232, ISSN 0065-1281.

Gruys, E.; Toussaint, M.J.M.; Niewold, T.A., \& Koopmans, S.J. (2005). Acute Phase Reaction and Acute Phase Proteins. Journal of Zhejiang University Science B, Vol.6, No.11, (November 2005), pp. 1045-1056, ISSN 1862-1783

Gundry, R.L.; Fu, Q.; Jelinek, C.K.; Van Eyk, J.E. \& Cotter R.J. (2007). Investigation of an Albumin-enriched Fraction of Human Serum and its Albuminome. Proteomics Clinical Applications, Vol.1, No.1, (January 1), pp. 73-88, ISSN 1862-8354

Hartmann, K.; Henz, B.M.; Krüger-Krasagakes, S.; Köhl, J.; Burger, R.; Guhl, S.; Haase, I.; Lippert, U., \& Zuberbier, T. (1997). C3a and C5a Stimulate Chemotaxis of Human Mast Cells. Blood, Vol.89, No.8, (April 15), pp. 2863-2870, ISSN 1528-0020

Hoffman, S.A.; Joo, W.A.; Echan, L.A. \& Speicher, D.W. (2007). Higher Dimensional (Hi-D) Separation Atrategies Dramatically Improve the Potential for Cancer Biomarker Detection in Serum and Plasma. Journal of Chromatography. B, Analytical Technologies 
in The Biomedical and Life Sciences, Vol.849, No.1-2, (April 2007), pp. 43-52, ISSN 0021-9673

Hongsachart, P.; Huang-Liu, R.; Sinchaikul, S.; Pan, F.M.; Phutrakul, S.; Chuang, Y.M.; Yu, C.J. \& Chen, S.T. (2009). Glycoproteomic Analysis of WGA-bound Glycoprotein Biomarkers in Sera from Patients with Lung Adenocarcinoma. Electrophoresis, Vol.30, No.7, (April 2009), pp. 1206-1220, ISSN 1522-2683

Huber, A.R.; Kunkel S.L.; Todd 3rd, R.F., \& Weiss, S.J. (1991). Regulation of Transendothelial Neutrophil Migration by Endogenous Interleukin-8. Science, Vol.254, No.5028, (October 4), pp. 99-102, ISSN 1095-9203

Hunt, S.M.; Thomas, M.R.; Sebastian, L.T.; Pedersen, S.K.; Harcourt, R.L.; Sloane, A.J. \& Wilkins, M.R. (2005). Optimal Replication and the Importance of Experimental Design for Gel-based Quantitative Proteomics. Journal of Proteome Research, Vol.4, No.3, (May-June 2005), pp. 809-819, ISSN 1535-3907

Imhof, B.A. \& Aurrand-Lions, M. (2004). Adhesion Mechanisms Regulating the Migration of Monocytes. Nature Reviews Immunology, Vol.4, No.6, (June 2004), pp. 432-444, ISSN 1474-1741

Kadowaki, M.; Sangai, T.; Nagashima, T.; Sakakibara, M.; Yoshitomi, H.; Takano, S.; Sogawa, K.; Umemura, H.; Fushimi, K.; Nakatani, Y.; Nomura, F. \& Miyazaki, M. (2011). Identification of Vitronectin as a Novel Serum Marker for Early Breast Cancer Detection Using a New Proteomic Approach. Journal of Cancer Research and Clinical Oncology, (January 2011), DOI: 10.1007/s00432-010-0974-9, ISSN 1432-1335

Kang, U.B.; Yeom, J.; Kim, H. \& Lee, C. (2010). Quantitative Analysis of mTRAQ-labeled Proteome Using Full MS Scans. Journal of Proteome Research, Vol.9, No.7, (July 2010), pp. 3750-3758, ISSN 1535-3907

Kawai, T.; Seki, M.; Hiromatsu, K.; Eastcott, J.W.; Watts, G.F.; Sugai, M.; Smith, D.J.; Porcelli, S.A., \& Taubman, M.A. (1999). Selective Diapedesis of Th1 Cells Induced by Endothelial Cell RANTES. Journal of Immunology, Vol.163, No.6, (September 1999), pp. 3269-3278, ISSN 1550- 6606

Kinashi, T. (2005). Intracellular Signalling Controlling Integrin Activation in Lymphocytes. Nature Reviews Immunology, Vol.5, No.7 (July 2005), pp. 546-559, ISSN 1474-1741

Kolla, V.; Jenö, P.; Moes, S.; Tercanli, S.; Lapaire, O.; Choolani, M. \& Hahn, S. (2010). Quantitative Proteomics Analysis of Maternal Plasma in Down Syndrome Pregnancies Using Isobaric Tagging Reagent (iTRAQ). Journal of Biomedicine and Biotechnology, (November 2009), doi:10.1155/2010/952047, ISSN 1110-7251

Konsman, J.P.; Parnet, P. \& Dantzer, R. (2002). Cytokine-induced Sickness Behaviour: Mechanisms and Implications. Trends in Neurosciences, Vol.25, No.3, (March 2002), pp. 154-159, ISSN 0166-2236

Konya, V.; Sturm, E.M.; Schratl, P.; Beubler, E.; Marsche, G.; Schuligoi, R.; Lippe, I.Th.; Peskar, B.A. \& Heinemann, A. (2010). Endothelium-derived Prostaglandin I(2) Controls the Migration of Eosinophils. The Journal of Allergy and Clinical Immunology, Vol.125, No.5, (May 2010), pp. 1105-1113, ISSN 0091-6749

Krueger, J.M.; Walter, J.; Dinarello, C.A.; Wolff, S.M. \& Chedid, L. (1984). Sleep-promoting Effects of Endogenous Pyrogen (Interleukin-1). American Journal of Physiology Regulatory, Integrative and Comparative Physiology, Vol.246, No.6, (June 1984), pp. R994-R999, ISSN 1522-1490 
Kuehn, H.S.; Jung, M.Y.; Beaven, M.A.; Metcalfe, D.D. \& Gilfillan, A.M. (2011). Prostaglandin E2 Activates and Utilizes mTORC2 as a Central Signaling Locus for the Regulation of Mast Cell Chemotaxis and Mediator Release. The Journal of Biological Chemistry, Vol.286, No.1, (January 2011), pp. 391-402, ISSN 1083-351X

Langer, H.F. \& Chavakis, T. (2009). Leukocyte-endothelial Interactions in Inflammation. Journal of Cellular and Molecular Medicine, Vol.13, No.7, (July 2009), pp. 1211-1220, ISSN 1582-1838

Lasky, L.A. (1995). Selectin-carbohydrate Interactions and the Initiation of the Inflammatory Response. Annual Review of Biochemistry, Vol.64, pp. 113-139, ISSN 0066-4154

Lawrence, M.B. \& Springer, T.A. (1991). Leukocytes Roll on a Selectin at Physiologic Flow Rates: Distinction from and Prerequisite for Adhesion Through Integrins. Cell Vol.65, No.5, (May 1991), pp. 859-873, ISSN 0092-8674

Leonard, B.E. \& Song, C. (1999). Stress, Depression, and the Role of Cytokines. Advances in Experimental Medicine and Biology, Vol.461, pp. 251-265, ISSN 0065-2598

Ley, K.; Laudanna, C.; Cybulsky, M.I. \& Nourshargh, S. (2007). Getting to the Site of Inflammation: the Leukocyte Adhesion Cascade Updated. Nature Reviews Immunology, Vol.7, No.9, (September 2007), pp. 678-689, ISSN 1474-1741

Libby, P. (2002). Inflammation in Atherosclerosis. Nature, Vol.420, No.6917, (December 2002), pp. 868-874, ISSN 1476-4687

Libby, P.; Okamoto, Y.; Rocha, V.Z. \& Folco, E. (2010) Inflammation in Atherosclerosis: Transition from Theory to Practice. Circulation Journal, Vol.74, No.2, (February 2010), pp. 213-220, ISSN 1347-4820

Lin, B.; White, J.T.; Wu, J.; Lele, S.; Old, L.J.; Hood, L. \& Odunsi, K. (2009). Deep Depletion of Abundant Serum Proteins Reveals Low-abundant Proteins as Potential Biomarkers for Human Ovarian Cancer. Proteomics Clinical Applications, Vol.3, No.7, (July 2009), pp. 853-861, ISSN 1862-8354

Lu, B.; Moser, A.H.; Shigenaga, J.K.; Feingold, K.R. \& Grunfeld, C. (2006). Type II Nuclear Hormone Receptors, Coactivator, and Target Gene Repression in Adipose Tissue in the Acute-phase Response. Journal of Lipid Research, Vol.47, No.10, (October 2006), pp. 2179-2190, ISSN 1539-7262

Luheshi, G.N.; Gardner, J.D.; Rushforth, D.A.; Loudon, A.S. \& Rothwell N.J. (1999). Leptin Actions on Food Intake and Body Temperature Are Mediated by IL-1. Proceedings of the National Academy of Sciences of the United States of America, Vol.96, No.12, (June 8), pp. 7047-7052, ISSN 1091-6490

Lundberg, I.E. \& Grundtman, C. (2008). Developments in the Scientific and Clinical Understanding of Inflammatory Myopathies. Arthritis Research \& Therapy, Vol.10, No.5, (October 2008), pp. 220, ISSN 1478-6362

Maseri, A.; Biasucci, L.M. \& Liuzzo, G. (1996). Inflammation in Ischaemic Heart Disease. British Medical Journal, Vol.312, No.7038, (April 1996), pp. 1049-1050, ISSN 14685833

McGuirk, J.; Hao, G.; Hou, W.; Abhyankar, S.; Williams, C.; Yan, W.; Yuan, J.; Guan, X.; Belt, R.; Dejarnette, S.; Wieman, J. \& Yan, Y. (2009). Serum Proteomic Profiling and Haptoglobin Polymorphisms in Patients with GVHD after Allogeneic Hematopoietic Cell Transplantation. Journal of Hematolology and Oncology, Vol.2, No.17, (April 2009), doi:10.1186/1756-8722-2-17, ISSN: 1756-8722 
Mizel, S.B.; Dayer, J.M.; Krane, S.M. \& Mergenhagen S.E. (1981). Stimulation of Rheumatoid Synovial Cell Collagenase and Prostaglandin Production by Partially Purified Lymphocyte-activating Factor (Interleukin 1). Proceedings of the National Academy of Sciences of the United States of America, Vol.78, No.4, (April 1981), pp. 2474-2477, ISSN 1091-6490

Mortensen, R.F.; Shapiro, J.; Lin, B.F.; Douches, S. \& Neta, R. (1988). Interaction of Recombinant IL-1 and Recombinant Tumor Necrosis Factor in the Induction of Mouse Acute Phase Proteins. Journal of Immunology, Vol.140, No.7, (April 1988), pp. 2260-2266, ISSN 1550-6606

Muraguchi, A.; Hirano, T.; Tang, B.; Matsuda, T.; Horii, Y.; Nakajima, K. \& Kishimoto, T. (1988). The Essential Role of B cell Stimulatory Factor 2 (BSF-2/IL-6) for the Terminal Differentiation of B cells. The Journal of Experimental Medicine, Vol.167, No.2, (February 1988), pp. 332-344, ISSN 1540-9538

Murakoshi, Y.; Honda, K.; Sasazuki, S.; Ono, M.; Negishi, A.; Matsubara, J.; Sakuma, T.; Kuwabara, H.; Nakamori, S.; Sata, N.; Nagai, H.; Ioka, T.; Okusaka, T.; Kosuge, T.; Shimahara, M.; Yasunami, Y.; Ino, Y.; Tsuchida, A.; Aoki, T.; Tsugane, S. \& Yamada, T. (2011). Plasma biomarker discovery and validation for colorectal cancer by quantitative shotgun mass spectrometry and protein microarray. Cancer Science, Vol.102, No.3, (March 2011), pp. 630-638, ISSN 1349-7006

Murooka, T.T.; Rahbar, R.; Platanias, L.C. \& Fish, E.N. (2008). CCL5-mediated T-cell Chemotaxis Involves the Initiation of mRNA Translation Through mTOR/4E-BP1. Blood, Vol.111, No.10, (May 2008), pp. 4892-4901, ISSN 1528-0020

Nawroth, P.P.; Handley, D.A.; Esmon, C.T. \& Stern, D.M. (1986). Interleukin 1 Induces Endothelial Cell Procoagulant while Suppressing Cell-surface Anticoagulant Activity. Proceedings of the National Academy of Sciences of the United States of America, Vol.83, No.10, (May 1986), pp. 3460-3464, ISSN 1091-6490

Okusawa, S.; Yancey, K.B.; van der Meer, J.W.; Endres, S.; Lonnemann, G.; Hefter, K.; Frank, M.M.; Burke, J.F.; Dinarello, C.A. \& Gelfand, J.A. (1988). C5a Stimulates Secretion of Tumor Necrosis Factor from Human Mononuclear Cells In Vitro. Comparison with Secretion of Interleukin 1 Beta and Interleukin 1 Alpha. The Journal of Experimental Medicine 168, No.1, (July 1988), pp. 443-448, ISSN 1540-9538

Omenn, G.S.; Menon, R.; Adamski, M.; Blackwell, T.; Haab, B.B.; Gao, W. \& States, D.J. (2007) The Human Plasma and Serum Proteome, In: Proteomics of Human Body Fluids: Principles, Methods, and Applications, V. Thongboonkerd., (Ed.),195-224, Humana Press Inc, ISBN 978-1-58829-657-3,Totowa, NJ.

Opp, M.R. \& Krueger, J.M. (1991). Interleukin 1-receptor Antagonist Blocks Interleukin 1Induced Sleep and Fever. American Journal of Physiology - Regulatory, Integrative and Comparative Physiology, Vol.260, No.2, (February 1991), pp. R453 -R457, ISSN 15221490

Palma, A.S.; De Carvalho, M.; Grammel, N.; Pinto, S.; Barata, N.; Conradt, H.S. \& Costa, J. (2008). Proteomic Analysis of Plasma from Portuguese Patients with Familial Amyotrophic Lateral Sclerosis. Amyotrophic Lateral Sclerosis, Vol.9, No.6, (December 2008), pp. 339-349, ISSN 1748-2968

Park, J.Y.; Mun, J.H.; Lee, B.H.; Heo, S.H.; Kim, G.H. \& Yoo, H.W. (2009). Proteomic Analysis of Sera of Asymptomatic, Early-stage Patients with Wilson's Disease. 
Proteomics Clinical Applications, Vol.3, No.10, (October 2009), pp. 1185-1190, ISSN 1862-8354

Pate, M.; Damarla, V.; Chi, D.S.; Negi, S. \& Krishnaswamy, G. (2010). Endothelial Cell Biology: Role in the Inflammatory Response. Advances in Clinical Chemistry, Vol.52, pp. 109-130, ISSN 0065-2423

Plomgaard, P.; Fischer, C.P.; Ibfelt, T.; Pedersen, B.K. \& van Hall, G. (2008). Tumor Necrosis Factor-\{alpha\} Modulates Human in Vivo Lipolysis. Journal of Clinical Endocrinolology \& Metabolism, Vol.93, No.2, (February 2008), pp. 543-549, ISSN 19457197

Prowse, K.R. \& Baumann H. (1989). Interleukin-1 and Interleukin-6 Stimulate Acute-phase Protein Production in Primary Mouse Hepatocytes. Journal of Leukocyte Biology, Vol.45, No.1, (January 1989), pp. 55-61, ISSN 0741-5400

Qian, W.J.; Petritis, B.O.; Kaushal, A.; Finnerty, C.C.; Jeschke, M.G.; Monroe, M.E.; Moore, R.J.; Schepmoes, A.A.; Xiao, W.; Moldawer, L.L.; Davis, R.W.; Tompkins, R.G.; Herndon, D.N.; Camp, D.G. 2nd \& Smith, R.D. (2010). Inflammation and the Host Response to Injury Large Scale Collaborative Research Program. Plasma Proteome Response to Severe Burn Injury Revealed by ${ }^{18} \mathrm{O}-$ labeled "Universal" ReferenceBased Quantitative Proteomics. Journal of Proteome Research, Vol.9, No.9, (September 2010), pp. 4779-4789, ISSN 1535-3907

Ramadori, G. \& Christ, B. (1999). Cytokines and the Hepatic Acute-phase Response. Seminars in Liver Disease, Vol.19, No.2, pp. 141-155, ISSN 0272-8087

Roelofsen, H.; Alvarez-Llamas, G.; Dijkstra, M.; Breitling, R.; Havenga, K.; Bijzet, J.; Zandbergen, W.; de Vries, M.P.; Ploeg, R.J. \& Vonk, R.J. (2007). Analyses of Intricate Kinetics of the Serum Proteome During and After Colon Surgery by Protein Expression Time Series. Proteomics, Vol.7, No.17, (September 2007), pp. 3219-3228, ISSN 1615-9681

Rothwell, N.J. \& Hopkins S.J. (1995). Cytokines and the Nervous System II: Actions and Mechanisms of Action. Trends in Neurosciences, Vol.18, No.3, (March 1995), pp. 130136, ISSN 0166-2236

Rydén, M.; Dicker, A.; van Harmelen, V.; Hauner, H.; Brunnberg, M.; Perbeck, L.; Lönnqvist, F. \& Arner P. (2002). Mapping of Early Signaling Events in Tumor Necrosis Factora-mediated Lipolysis in Human Fat Cells. Journal of Biological Chemistry, Vol.277, No.2, (January 2002), pp. 1085-1091, ISSN 1083-351X

Ryu, J.; Lee, S.R.; Park, S.G.; Kang, S.; Kim, H.J. \& Park, B.C. (2010). Change in Serum Proteome During Allogeneic Hematopoietic Stem Cell Transplantation and Clinical Significance of Serum C-reactive Protein and Haptoglobin. Experimental and Molecular Medicine, Vol.42, No.9, (September 2010), pp. 651-661, ISSN 1226-3613

Saldova, R.; Royle, L.; Radcliffe, C.M.; Abd Hamid, U.M.; Evans, R.; Arnold, J.N.; Banks, R.E.; Hutson, R.; Harvey, D.J.; Antrobus, R.; Petrescu, S.M.; Dwek, R.A. \& Rudd, P.M. (2007). Ovarian Cancer is Associated with Changes in Glycosylation in Both Acute-phase Proteins and IgG. Glycobiology, Vol.17, No.12, (December 2007), pp. 1344-1357, ISSN 1460-2423

Salgado, F.J.; Vázquez, S.; Iglesias, A.; Pérez-Díaz, A.; Mera-Varela, A.; Arias, P. \& Nogueira, M. (2010). Application of Thiophilic Chromatography to Deplete Serum Immunoglobulins in Sample Preparation for Bidimensional Electrophoresis. Analytica Chimica Acta, Vol.658, No.1, (January 2010), pp. 18-31, ISSN 003-2670 
Salvatore, A.; Cigliano, L.; Bucci, E.M.; Corpillo, D.; Velasco, S.; Carlucci, A.; Pedone, C. \& Abrescia, P. (2007). Haptoglobin Binding to Apolipoprotein A-I Prevents Damage from Hydroxyl Radicals on its Stimulatory Activity of the Enzyme LecithinCholesterol Acyl-transferase. Biochemistry, Vol.46, No.39, (October 2007), pp. 1115811168, ISSN 0006-2960

Sapolsky, R.; Rivier, C.; Yamamoto, G.; Plotsky, P. \& Vale, W. (1987). Interleukin-1 Stimulates the Secretion of Hypothalamic Corticotropin-releasing factor. Science, Vol.238, No.4826, (October 1987), pp. 522-524, ISSN 1095-9203

Schenkel, A.R., Mamdouh, Z. \& Muller W.A. (2004). Locomotion of Monocytes on Endothelium is a Critical Step during Extravasation. Nature Immunology, Vol.5, No. 4, (April 2004), pp. 393-400, ISSN 1529-2916

Scott, D.L.; Wolfe, F. \& Huizinga, T.W. (2010). Rheumatoid Arthritis. Lancet, Vol.376, No.9746, (September 2010), pp. 1094-108, ISSN 0140-6736

Serada, S.; Fujimoto, M.; Ogata, A.; Terabe, F.; Hirano, T.; Iijima, H.; Shinzaki, S.; Nishikawa, T.; Ohkawara, T.; Iwahori, K.; Ohguro, N.; Kishimoto, T. \& Naka, T. iTRAQ-based Proteomic Identification of Leucine-rich Alpha-2 Glycoprotein as a Novel Inflammatory Biomarker in Autoimmune Diseases. Annals of Rheumatic Diseases, Vol.69, No.4, (April 2010), pp. 770-774, ISSN 1468-2060

Seriramalu, R.; Pang, W.W.; Jayapalan, J.J.; Mohamed, E.; Abdul-Rahman, P.S.; Bustam, A.Z.; Khoo, A.S. \& Hashim, O.H. (2010). Application of Champedak Mannosebinding Lectin in the Glycoproteomic Profiling of Serum Samples Unmasks Reduced Expression of Alpha-2 Macroglobulin and Complement Factor B in Patients with Nasopharyngeal Carcinoma. Electrophoresis, Vol.31, No.14, (July 2010), pp. 2388-2395, ISSN 1522-2683

Stefferl, A.; Hopkins, S.J.; Rothwell, N.J. \& Luheshi, G.N. (1996). The Role of TNF-alpha in Fever: Opposing Actions of Human and Murine TNF-alpha and Interactions with IL-beta in the Rat. British Journal of Pharmacology, Vol.118, No.8, (August 1996), pp. 1919-1924, ISSN 1476-5381

Sussulini, A.; Dihazi, H.; Banzato, C.E.; Arruda, M.A.; Stühmer, W.; Ehrenreich, H.; Jahn, O. \& Kratzin, H.D. (2011). Apolipoprotein A-I as a Candidate Serum Marker for the Response to Lithium Treatment in Bipolar Disorder. Proteomics, Vol.11, No.2, (January 2011), pp. 261-269, ISSN 1615-9861

Taga, T.; Kawanishi, Y.; Hardy, R.R.; Hirano, T. \& Kishimoto, T. (1987). Receptors for B Cell Stimulatory Factor 2. Quantitation, Specificity, Distribution, and Regulation of their Expression. The Journal of Experimental Medicine, Vol.166, No.4, (October 1987), pp. 967-981, ISSN 1540-9538

Theiss, A.L.; Simmons, J.G.; Jobin, C. \& Lund P.K. (2005). Tumor Necrosis Factor (TNF) Alpha Increases Collagen Accumulation and Proliferation in Intestinal Myofibroblasts via TNF Receptor 2. The Journal of Biological Chemistry, Vol.280, No.43 (October 2005), pp. 36099-36109, ISSN 1083-351X

Tocco-Bradley, R.; Georgieff, M.; Jones, C.T.; Moldawer, L.L.; Dinarello, C.A.; Blackburn, G.L. \& Bistrian B.R. (1987). Changes in Energy Expenditure and Fat Metabolism in Rats Infused with Interleukin-1. European Journal of Clinical Investigation, Vol.17, No.6, (December 1987), pp. 504-510, ISSN 1365-2362

Tracey, K.J.; Morgello, S.; Koplin, B.; Fahey 3rd, T.J.; Fox, J.; Aledo, A.; Manogue, K.R. \& Cerami, A. (1990). Metabolic Effects of Cachectin/Tumor Necrosis Factor are 
Modified by Site of Production. Cachectin/Tumor Necrosis Factor-secreting Tumor in Skeletal Muscle Induces Chronic Cachexia, while Implantation in Brain Induces Predominantly Acute Anorexia. The Journal of Clinical Investigation, Vol.86, No.6, (December 1990), pp. 2014-2024, ISSN 1558-8238

van Miert, A.S. (1995). Pro-inflammatory Cytokines in a Ruminant Model: Pathophysiological, Pharmacological, and Therapeutic Aspects. The Veterinary Quarterly, Vol.17, No.2, (June 1995), pp. 41-50, ISSN 0165-2176

Vassalli, P. (1992). The Pathophysiology of Tumor Necrosis Factors. Annual Review of Immunology, Vol.10, pp. 411-452, ISSN 0036-4665

Vestweber, D. (2007). Adhesion and Signaling Molecules Controlling the Transmigration of Leukocytes through Endothelium. Immunological Reviews, Vol.218, (August 2007), pp. 178-196, ISSN 1600-065X

Wan, C.; La, Y.; Zhu, H.; Yang, Y.; Jiang, L.; Chen, Y.; Feng, G.; Li, H.; Sang, H.; Hao, X.; Zhang, G. \& He, L. (2007). Abnormal Changes of Plasma Acute Phase Proteins in Schizophrenia and the Relation between Schizophrenia and Haptoglobin (Hp) Gene. Amino Acids, Vol. 32, No.1, (January 2007), pp. 101-108, ISSN 1438-2199

Wan, J.; Sun, W.; Li, X.; Ying, W.; Dai, J.; Kuai, X.; Wei, H.; Gao, X.; Zhu, Y.; Jiang, Y.; Qian, X.\& He, F. (2006). Inflammation Inhibitors were Remarkably Up-regulated in Plasma of Severe Acute Respiratory Syndrome Patients at Progressive Phase. Proteomics, Vol.6, No.9, (May 2006), pp. 2886-2894, ISSN 1615-9861

Watanabe, J.; Grijalva, V.; Hama, S.; Barbour, K.; Berger, F.G.; Navab, M.; Fogelman, A.M. \& Reddy, S.T. (2009). Hemoglobin and Its Scavenger Protein Haptoglobin Associate with ApoA-1-containing Particles and Influence the Inflammatory Properties and Function of High Density Lipoprotein. Journal of Biological Chemistry, Vol.284, No.27, (July 2009), pp. 18292-18301, ISSN 0021-9258

Wiesner, C.; Hannum, C.; Reckamp, K.; Figlin, R.; Dubridge, R.; Roy, S.M.; Lin, S.; Becker, C.H.; Jones, T.; Hiller, J.; Cheville, J.C. \& Wilson, K. (2010). Consistency of a two clinical site sample collection: a proteomics study. Proteomics Clinical Applications, Vol.4, No.8-9 (September 2010), pp. 726-738, ISSN 1862-8354

Xanthoulea, S.; Pasparakis, M.; Kousteni, S.; Brakebusch, C.; Wallach, D.; Bauer, J.; Lassmann, H. \& Kollias, G. (2004). Tumor Necrosis Factor (TNF) Receptor Shedding Controls Thresholds of Innate Immune Activation that Balance Opposing TNF Functions in Infectious and Inflammatory Diseases. The Journal of Experimental Medicine, Vol.200, No.3, (August 2004), pp. 367-376, ISSN 1540-9538

Yang, M.H.; Tyan, Y.C.; Jong, S.B.; Huang, Y.F.; Liao, P.C.\& Wang, M.C. (2007). Identification of Human Hepatocellular Carcinoma-related Proteins by Proteomic Approaches. Analytical and Bioanalytical Chemistry, Vol.388, No.3, (June 2007), pp. 637-643, ISSN 1618-2650

Zhao, Y.; He, X.; Shi, X.; Huang, C.; Liu, J.; Zhou, S. \& Heng, C.K. (2010). Association between Serum Amyloid A and Obesity: a Meta-analysis and Systematic Review. Inflammation Research, Vol.59, No.5, (May 2010), pp. 323-334, ISSN 1420-908X 
ACUTE PHASE PROTEINS AS EARIY NON-SPECIFIC BIOMARKERS OF HUMAN ANO VETERINARY DISEASES

Edited by Francheo Vees

\section{Acute Phase Proteins as Early Non-Specific Biomarkers of Human and Veterinary Diseases}

Edited by Prof. Francisco Veas

ISBN 978-953-307-873-1

Hard cover, 408 pages

Publisher InTech

Published online 10, October, 2011

Published in print edition October, 2011

The two volumes of Acute Phase Proteins book consist of chapters that give a large panel of fundamental and applied knowledge on one of the major elements of the inflammatory process during the acute phase response, i.e., the acute phase proteins expression and functions that regulate homeostasis. We have organized this book in two volumes - the first volume, mainly containing chapters on structure, biology and functions of APP, the second volume discussing different uses of APP as diagnostic tools in human and veterinary medicine.

\section{How to reference}

In order to correctly reference this scholarly work, feel free to copy and paste the following:

Francisco J. Salgado, Pilar Arias, Ana Canda-Sánchez and Montserrat Nogueira (2011). Acute Phase Proteins as Biomarkers of Disease: From Bench to Clinical Practice, Acute Phase Proteins as Early Non-Specific Biomarkers of Human and Veterinary Diseases, Prof. Francisco Veas (Ed.), ISBN: 978-953-307-873-1, InTech, Available from: http://www.intechopen.com/books/acute-phase-proteins-as-early-non-specific-biomarkers-ofhuman-and-veterinary-diseases/acute-phase-proteins-as-biomarkers-of-disease-from-bench-to-clinicalpractice

\section{INTECH}

open science | open minds

\section{InTech Europe}

University Campus STeP Ri Slavka Krautzeka 83/A 51000 Rijeka, Croatia Phone: +385 (51) 770447 Fax: +385 (51) 686166 www.intechopen.com

\author{
InTech China \\ Unit 405, Office Block, Hotel Equatorial Shanghai \\ No.65, Yan An Road (West), Shanghai, 200040, China \\ 中国上海市延安西路65号上海国际贵都大饭店办公楼405单元 \\ Phone: +86-21-62489820 \\ Fax: $+86-21-62489821$
}


(C) 2011 The Author(s). Licensee IntechOpen. This is an open access article distributed under the terms of the Creative Commons Attribution 3.0 License, which permits unrestricted use, distribution, and reproduction in any medium, provided the original work is properly cited. 